\title{
ASSESSMENT OF A TWO-CHANNEL \\ IMPLANTABLE PERONEAL NERVE STIMULATOR POST-STROKE
}

Anke I.R. Kottink-Hutten 
Address of correspondence:

Anke Kottink-Hutten

Roessingh Research and Development

PO Box 310

7500 AH Enschede

The Netherlands

$\mathrm{T}+31-(0) 53-4875733$

a.kottink@rrd.nl

Printed by Gildeprint Drukkerijen, Enschede, The Netherlands ISBN: 978-90-365-2959-4

(C) Copyright: A.I.R. Kottink-Hutten, Enschede, The Netherlands All rights reserved. No part of this publication may be reproduced, stored in a retrieval system, or transmitted, in any form and by any means, electronic, photocopying, or otherwise, without the permission of the author. 


\title{
ASSESSMENT OF A TWO-CHANNEL IMPLANTABLE PERONEAL NERVE STIMULATOR POST-STROKE
}

\author{
PROEFSCHRIFT \\ ter verkrijging van \\ de graad van doctor aan de Universiteit Twente, \\ op gezag van de rector magnificus, \\ Prof. dr. H. Brinksma, \\ volgens besluit van het College voor Promoties \\ in het openbaar te verdedigen \\ op vrijdag 12 februari 2010 om 16.45 uur \\ door \\ Anke Ida Roza Kottink-Hutten \\ geboren op 28 januari 1979 \\ te Geesteren (OV)
}


Dit proefschrift is goedgekeurd door de promotoren:

Prof. Dr. Ir. H.J. Hermens

Prof. Dr. M.J. IJzerman 


\section{Promotiecommissie}

\section{Voorzitter/secretaris}

Prof. Dr. Ir. A.J. Mouthaan

Universiteit Twente

\section{Promotoren}

Prof. Dr. Ir. H.J. Hermens

Universiteit Twente

Prof. Dr. M.J. IJzerman

Universiteit Twente

Leden

Prof. Dr. J.H. Burridge

Prof. Dr. G. Kwakkel

Prof. Dr. J.S. Rietman

Prof. Dr. Ir. P.H. Veltink

Dr. H.P.J. Buschman

University of Southampton

VU Medisch Centrum

Universiteit Twente

Universiteit Twente

Medtronic Nederland

\section{Paranimfen}

Martin Tenniglo

Janine van Til 
This research was supported by the European Eureka program, the Department of Dutch Ministry of Economic Affairs in The Hague, The Netherlands

\section{SenterNovem}

The publication of this thesis was generously supported by:

Roessingh Research and Development, Enschede

Roessingh Rehabilitation Center, Enschede

Chair Biomedical Signals and Systems, University of Twente, Enschede

Opus Medical, Belgium (www.opusmedical.eu)

Finetech Medical, United Kingdom (www.finetech-medical.co.uk)

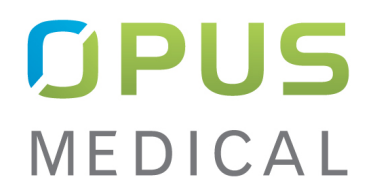

$\underset{\checkmark}{\text { Finetech Medical }}$ 


\section{Contents}

Chapter 1

Chapter 2

Chapter 3

Chapter 4

Chapter 5

Chapter 6

Chapter 7

Summary

Samenvatting

Dankwoord

Over de auteur

Progress Range
General Introduction

The orthotic effect of functional electrical stimulation on the improvement of walking in stroke patients with a dropped foot: a systematic review

A randomized controlled trial of an implantable two-

channel peroneal nerve stimulator on walking speed and activity in post-stroke hemiplegia

Therapeutic effect of an implantable peroneal nerve

stimulator in subjects with chronic stroke and footdrop: a randomized controlled trial Effects of an implantable two-channel peroneal nerve stimulator versus conventional walking device on spatiotemporal parameters and kinematics of hemiparetic gait

Measuring quality of life in stroke subjects receiving an implanted neural prosthesis for drop foot

General Discussion 

Chapter 1

General Introduction 



\section{General Introduction}

Drop foot is a simple term that describes a rather complex problem. It can be defined as the inability to raise the foot during the swing phase of gait. A drop foot is a condition that is often found in Multiple Sclerosis (MS), incomplete spinal cord lesion (SCI) and most notably, stroke. In the Netherlands, the total population of patients with MS and SCI is respectively about 16,000 and 10,000 $(1,2)$. Each year 41,000 people are involved with a first stroke (2.5 stroke patients per 1,000 inhabitants) and 7,000 people with a second stroke. Nowadays about 190,000 patients live with the consequences of a stroke (3). In the European Union (EU), the annual incidence is approximately 2 per 1,000 inhabitants, but this incidence increases exponentially with age (e.g. 10 per 1,000 65-74 year old males, 20 per 1,000 75-84 year old males, etc.). Due to the ageing population in the EU the number of incidents is therefore increasing dramatically, and the incidence is believed to double over the next 50 years (4). So, stroke is a major illness in Western countries and it results in huge disabling consequences. It causes impairment of the cognitive, sensory, perceptive and motor functions.

The ultimate goal in the rehabilitation of stroke survivors is optimal recovery of motor functions to regain independence, and hence, to improve health-related quality of life (5). To reach this goal, regaining the ability to walk again is very important (6). However, the occurrence of a drop foot in stroke survivors may hamper full recovery of the walking ability.

Drop foot following stroke is thought to be caused mainly by poor active control of the anterior tibial muscle combined with increased and inappropriate tone in the dorsal muscles of the leg, particularly the calf (7). The dorsiflexors assist in clearing the foot during swing phase and control plantar flexion of the foot upon heel strike. Plantar flexors provide a controlled roll-off, actively provide forward progression or push-off and accelerate the leg into swing. Walking becomes a challenge when the patient is not able to control the movements at the ankle. In fact, due to predominance of extensor synergy, hip and knee flexion are usually both reduced which further lengthens the limb functionally. As a result, the patient's gait is characterised by hip hitching and circumduction, to compensate for the extra length of the affected leg. Circumduction is the most energy efficient, although still inefficient, and most commonly observed compensation of stroke patients to correct their drop foot (8). A drop foot results in an inefficient and unstable gait pattern with a low walking speed, pain in the joints and 
muscles around the hip and a higher chance of stumbling and falling (9). Treating a drop foot in an adequate way is therefore an important element in stroke rehabilitation.

In the past, several solutions have been developed for the treatment of a drop foot. The conventional management of a drop foot is an ankle-foot orthosis (AFO), to maintain a neutral fixed position of the ankle with respect to the lower leg. Both rigid or more flexible AFOs can be prescribed in clinical practice. Rigid AFOs are usually prescribed to more severely affected stroke subjects, who also do not have enough ankle stability in stance, because of varus deformity. Less affected stroke subjects more often use flexible AFOs. However, there is only a limited to moderate level of evidence that AFOs improve hemiplegic gait parameters, due to lack of rigorously controlled trials (10). Also, results regarding the effect of AFOs on walking and balance control are very contradictory, which may be explained by differences in subject inclusion and study designs (11). The use of an AFO has major limitations, being both uncomfortable and awkward to use (12-14). AFOs can only be worn in shoes, and often the shoe with the AFO must be larger in size than that of the other foot (14). As a result, the AFO is often rejected by patients $(13,15)$. This is in accordance with other studies that showed that in clinical practice the non-use of shoe inserts and orthopaedic shoes varies from $8 \%$ to 75\% (16-22). Despite the limitations of AFOs, they also have some advantages. The stability given by the AFO counteracts hyperextension of the knee in stance, when present, and corrects a drop foot and varus deformity at the ankle during swing/ and or stance. It is also very simple to use and its cost is relatively low. Therefore, AFOS are probably the most commonly used treatment for drop foot today (14).

In 1961, a new method for correction of drop foot by means of electrical stimulation was introduced by Liberson (23). The stimulation was applied via electrodes on the skin and was synchronised with the gait cycle by a heel-switch worn in the shoe. Stimulation was turned on when the heel was lifted at the beginning of the swing phase. It then produced dorsiflexion of the ankle joint. Stimulation was turned off when the heel was placed on the floor again. For functional electrical stimulation (FES) to be effective both the nerve and the muscle supplied by the nerve must be undamaged. Therefore FES can only be used in conditions where the paralysis is due to an upper motor neuron lesion, so a lesion that occurs in the brain or spinal cord at or above the level of T12. In addition, severely affected drop foot subjects with a high degree of ankle instability during stance are usually also not suitable for using FES, because no 
support is given at stance. Besides providing ankle dorsiflexion during swing, the use of FES also results in a safe initial loading response in the stance phase.

A number of (theoretical) advantages of FES, in comparison with an orthosis, can be mentioned. FES allows both greater passive and active movement of the ankle, promoting proprioceptive input that is essential for postural control. It enables foot adaptation to uneven terrains, whereas the AFO restricts this adaptation because of its firm structure. In addition, there is evidence that stimulation of the common peroneal nerve may trigger knee and hip flexion and thus facilitate the flexion pattern needed for foot clearance during swing (14). Merletti suggests that this avoidance of compensatory movements implies a more energy efficient use of the hip and knee muscles (24). Other potential benefits of FES are prevention of disuse atrophy, increased local blood flow, better cosmetic appearance, reduced weight and muscle re-education $(14,25)$. However, the use of a transcutaneous stimulator have a number of shortcomings that limit its acceptance by patients $(26,27)$. These shortcomings are a lack of selectivity over the muscles to be activated, sensitivity of muscle response to electrode placement and pain and tissue irritation associated with passage of current through the skin. In addition, the donning of a transcutaneous FES system requires considerable more time compared to donning of an orthosis, as it requires a rather precise placement of surface electrodes. This is especially an issue when FES is used by stroke patients as they often have one fully functional hand.

Recognition of these limitations has led to a second approach that uses implantable electrodes. Assuming that the drop foot requires a permanent solution, an implantable FES system might be considered. Several implantable FES systems have been developed in the past (28-30). The first generation of implants consisted of a single channel stimulation system, where the electrodes were attached to the common peroneal nerve. The advantages of this approach are that it eliminates the passage of current through the skin and reduces transmitter-positioning problems, which improves the convenience for the user. However, this one-channel system failed to solve the selectivity problem, since it did not allow for differential activation of peroneus and anterior tibial muscles for inversion-eversion balance post surgery. Also it was not possible to control the physiological fluctuations of the desired dorsiflexion and eversion levels in every day life. To solve these problems, an implantable device with two independent channels 
was developed in the European Impulse project (31). This system stimulates the two main branches of the peroneal nerve separately in order to restore the ability to raise the foot during the swing phase of gait and allows balance of dorsiflexion and eversion. The stimulation levels are tuned manually on the basis of the perception of the patient. The onset of stimulation in this system is controlled by a heel switch.

Although the concept of FES for drop foot exists now for more than 40 years, it's clinical effects have not been addressed in high quality scientific studies. Literature is mainly based on case studies, uncontrolled trials and retrospective reviews (28,32-38). At present, only 1 randomized clinical trial (RCT) is published in which the effect of common peroneal nerve stimulation by a surface stimulator was examined (39). Burridge et al. made a comparison between walking with and without stimulation and concluded that patients could walk faster and more energy efficient with the peroneal nerve stimulator. Moreover, they recommend that future studies should compare peroneal nerve stimulation (PNS) with conventional splinting. For the development of evidence based stroke rehabilitation regarding the correction of drop foot it is necessary that better studies, using more profound methodological concepts, should be performed in future.

\section{Thesis outline and aims}

To progress towards evidence based application of PNS to improve lower extremity function, the aim of the present thesis is to evaluate an implantable two-channel peroneal nerve stimulator versus conventional splinting as a treatment option for chronic stroke patients with a dropped foot.

In chapter 2 a systematic review is described on the available evidence on the improvement of walking in stroke patients with a dropped foot when using PNS. Trials were identified that investigated the orthotic effect of FES on walking. All included studies were scored on methodological quality and studies that measured walking speed were pooled and a pooled difference including confidence interval was calculated.

The review concludes that PNS seems to have a positive orthotic effect on walking speed in stroke patients with a dropped foot. 
The largest part of this $\mathrm{PhD}$ thesis (chapters 3-6) is dedicated to a RCT comparing implantable PNS versus conventional treatment, consisting of the use of an AFO, orthopaedic shoes or no walking device, for the correction of a drop foot.

Chapter 3 reports the results of the RCT in which the orthotic effect of an implantable two-channel peroneal nerve stimulator, in comparison with the conventional treatment, on walking speed and daily activities was examined. The results were compared with the scientific literature to report the clinical relevance of the FES treatment.

Besides an orthotic effect, a therapeutic or carry-over effect of FES has been described in the literature. This therapeutic effect is defined by a temporary increase in voluntary control observed immediately after electrical stimulation. In chapter $\mathbf{4}$ the question is addressed whether there is a therapeutic effect in case of an implantable two-channel peroneal nerve stimulator after 6 months use. This was assessed by measuring the maximum value of the root mean square (RMS) of four lower limb muscles of the affected leg with both flexed and extended knee, by measuring walking speed and by measuring the muscle activity of the tibialis anterior muscle of the affected leg during the swing phase of gait.

The trial described in chapter $\mathbf{5}$ investigates if there is a surplus value of implantable PNS over the conventional treatment after a follow-up period of 6 months in chronic stroke subjects with a drop foot with regard to their walking pattern. By means of 3dimensional gait analysis (using Vicon ${ }^{\circledR}$ ) different spatiotemporal parameters and hip, knee and ankle ROM of both the paretic and non-paretic leg were recorded.

Chapter 6 addresses the question whether the treatment of a drop foot by means of an implantable two-channel peroneal nerve stimulator improves patients' health-related quality of life. Different health-related quality of life (HRQoL) instruments were included in the study and responsiveness to detect changes was investigated.

Finally, chapter 7 presents a general discussion on the implications of the scientific work of the present thesis for daily clinical practice. 


\section{References}

1. Publication of the Dutch Multiple Sclerosis Research Foundation (in Dutch: Stichting MS Research) 2009; http://www.msresearch.nl/index.php?content= content\&description1= Wat\%20is\%20MS?\&menu_id=2\&subtype=1\&expand=2.

2. van Asbeck FWA, redacteur. Handboek dwarslaesie revalidatie. Houten: Bohn, Stafleu, Van Loghum; 1998 (in Dutch).

3. Bots ML, van Dis SJ. Factsheet Stroke (in Dutch: Cijfers en feiten - Beroerte). Publication of the Dutch Heart Foundation (in Dutch: Nederlandse Hartstichting) 2006; http://www.hartstichting.nl/Uploads/Brochures/Factsheet\%20 Beroerte.pdf.

4. Integrating Information from Molecule to Man: Knowledge Discovery Accelerates Drug Development and Personalized Treatment in Acute Stroke (I-Know) 2008; http://www.iknow-stroke.eu/menu7-en.

5. Kwa VI, Limburg M, de Haan RJ. The role of cognitive impairment in the quality of life after ischaemic stroke. J Neurol 1996 Aug;243(8):599-604.

6. Mortimer D, Segal L. Comparing the incomparable? A systematic review of competing techniques for converting descriptive measures of health status into QALY-weights. Med Decis Making 2008 Feb 28;28(1):66-89.

7. Burridge JH, Wood DE, Taylor PN, McLellan DL. Indices to describe different muscle activation patterns, identified during treadmill walking, in people with spastic drop-foot. Med Eng Phys 2001 Jul;23(6):427-34.

8. Bogey R. Gait analysis. eMedicine 2009; http://emedicine.medscape.com/article/320160overview.

9. Bult JR, Hunink MG, Tsevat J, Weinstein MC. Heterogeneity in the relationship between the time tradeoff and Short Form-36 for HIV-infected and primary care patients. Med Care 1998 Apr;36(4):523-32.

10. Teasell RW, Foley NC, Bhogal SK, Speechley MR. An evidence-based review of stroke rehabilitation. Top Stroke Rehabil 2003 Spring;10(1):29-58.

11. Simons CD, van Asseldonk EH, van der Kooij H, Geurts AC, Buurke JH. Ankle-foot orthoses in stroke: effects on functional balance, weight-bearing asymmetry and the contribution of each lower limb to balance control. Clin Biomech (Bristol, Avon) 2009 Nov;24(9):769-75.

12. Stein RB, Chong S, Everaert DG, Rolf R, Thompson AK, Whittaker M, Robertson J, Fung J, Preuss R, Momose K, et al. A multicenter trial of a footdrop stimulator controlled by a tilt sensor. Neurorehabil Neural Repair 2006 Sep;20(3):371-9. 
13. Kenney LPJ, Bultstra G, Buschman R, Taylor P, Mann G, Hermens H, Holsheimer J, Nene A, Tenniglo $\mathrm{M}$, van der Aa H, Hobby J. A novel two-channel implanted drop foot stimulator: initial clinical results. Biomechanics of the Lower Limb in Health, Disease and Rehabilitation 2001; University of Salford, Salford:62-63.

14. Ring H, Treger I, Gruendlinger L, Hausdorff JM. Neuroprosthesis for footdrop compared with an ankle-foot orthosis: effects on postural control during walking. J Stroke Cerebrovasc Dis 2009 Jan;18(1):41-7.

15. Taylor PN, Burridge JH, Dunkerley AL, Lamb A, Wood DE, Norton JA, Swain ID. Patients' perceptions of the Odstock Dropped Foot Stimulator (ODFS). Clin Rehabil 1999 Oct;13(5):439-46.

16. Phillips B, Zhao H. Predictors of assistive technology abandonment. Assist Technol 1993;5(1):36-45.

17. Sykes L, Edwards J, Powell ES, Ross ER. The reciprocating gait orthosis: long-term usage patterns. Arch Phys Med Rehabil 1995 Aug;76(8):779-83.

18. Knowles EA, Boulton AJ. Do people with diabetes wear their prescribed footwear? Diabet Med 1996 Dec;13(12):1064-8.

19. Saag KG, Saltzman CL, Brown CK, Budiman-Mak E. The Foot Function Index for measuring rheumatoid arthritis pain: evaluating side-to-side reliability. Foot Ankle Int 1996 Aug;17(8):506-10.

20. Scherer MJ. Outcomes of assistive technology use on quality of life. Disabil Rehabil 1996 Sep;18(9):439-48.

21. Fransen M, Edmonds J. Off-the-shelf orthopedic footwear for people with rheumatoid arthritis. Arthritis Care Res 1997 Aug;10(4):250-6.

22. Reiber GE, Smith DG, Wallace C, Sullivan K, Hayes S, Vath C, Maciejewski ML, Yu O, Heagerty PJ, LeMaster J. Effect of therapeutic footwear on foot reulceration in patients with diabetes: a randomized controlled trial. JAMA 2002 May 15;287(19):2552-8.

23. Liberson WT, Homquest HJ, Scot D, Dow M. Functional electrotherapy: stimulation of the peroneal nerve synchronized with the swing phase of the gait of hemiplegic patients. Arch Phys Med Rehabil 1961 Feb;42:101-5.

24. Merletti R, Andina A, Galante M, Furlan I. Clinical experience of electronic peroneal stimulators in 50 hemiparetic patients. Scand J Rehabil Med 1979;11(3):111-21.

25. Pickard AS, Johnson JA, Feeny DH. Responsiveness of generic health-related quality of life measures in stroke. Qual Life Res 2005 Feb;14(1):207-19.

26. Rushton DN. Functional electrical stimulation. Physiol Meas 1997 Nov;18(4):241-75.

27. Taylor PN, Burridge JH, Dunkerley AL, Lamb A, Wood DE, Norton JA, Swain ID. Patients' perceptions of the Odstock Dropped Foot Stimulator (ODFS). Clin Rehabil 1999 Oct;13(5):439-46. 
28. Waters RL, McNeal D, Perry J. Experimental correction of footdrop by electrical stimulation of the peroneal nerve. J Bone Joint Surg Am 1975 Dec;57(8):1047-54.

29. Strojnik P, Acimovic R, Vavken E, Simic V, Stanic U. Treatment of drop foot using an implantable peroneal underknee stimulator. Scand J Rehabil Med 1987;19(1):37-43.

30. Haugland, M, Childs C, Ladouceur M, Haase J, Sinkjaer T. An implantable foot drop stimulator. $5^{\text {th }}$ Annual conference of IFESS 2000; Aalborg, Denmark:59-62.

31. van der Aa HE, Bultstra G, Verloop AJ, Kenney L, Holsheimer J, Nene A, Hermens HJ, Zilvold G, Buschman HP. Application of a dual channel peroneal nerve stimulator in a patient with a "central" drop foot. Acta Neurochir Suppl 2002;79:105-7.

32. Merletti R, Andina A, Galante M, Furlan I. Clinical experience of electronic peroneal stimulators in 50 hemiparetic patients. Scand J Rehabil Med 1979;11(3):111-21.

33. Bogataj U, Gros N, Kljajic M, Acimovic R, Malezic M. The rehabilitation of gait in patients with hemiplegia: a comparison between conventional therapy and multichannel functional electrical stimulation therapy. Phys Ther 1995 Jun;75(6):490-502.

34. Burridge J, Taylor P, Hagan S, Swain I. Experience of clinical use of the Odstock dropped foot stimulator. Artif Organs 1997 Mar;21(3):254-60.

35. Granat MH, Maxwell DJ, Ferguson AC, Lees KR, Barbenel JC. Peroneal stimulator; evaluation for the correction of spastic drop foot in hemiplegia. Arch Phys Med Rehabil 1996 Jan;77(1):19-24.

36. Stefanovska A, Gros N, Vodovnik L, Rebersek S, Acimovic-Janezic R. Chronic electrical stimulation for the modification of spasticity in hemiplegic patients. Scand J Rehabil Med Suppl 1988;17:115-21.

37. Glanz M, Klawansky S, Stason W, Berkey C, Chalmers TC. Functional electrostimulation in poststroke rehabilitation: a meta-analysis of the randomized controlled trials. Arch Phys Med Rehabil 1996 Jun;77(6):549-53.

38. Burridge JH SITP. Functional electrical stimulation: a review of the literature published on common peroneal nerve stimulation for the correction of dropped foot. Reviews in Clinical Gerontology 1998;8:155-61.

39. Burridge JH, Taylor PN, Hagan SA, Wood DE, Swain ID. The effects of common peroneal stimulation on the effort and speed of walking: a randomized controlled trial with chronic hemiplegic patients. Clin Rehabil 1997 Aug;11(3):201-10. 
Chapter 2

The orthotic effect of functional electrical stimulation on the improvement of walking in stroke patients with a dropped foot: a systematic review

Anke I.R. Kottink

Linda J.M. Oostendorp

Jaap H. Buurke

Anand V. Nene

Hermie J. Hermens

Maarten J. IJzerman

Artif Organs 2004 June;28(6):577-86.

Reprinted with permission 


\begin{abstract}
In the present study the available evidence on the improvement of walking in stroke patients with a dropped foot when using peroneal nerve stimulation was analysed.

A systematic review was performed to identify trials that investigated the orthotic effect of FES on walking in stroke patients with a dropped foot. Two independent raters scored the methodological quality of the included articles. Walking speed and PCI were selected as the primary outcome measures. Studies that measured walking speed were pooled and a pooled difference including confidence interval was calculated.

Eight studies were included in the review, of which one was a randomised controlled trial. Methodological score ranged from 8 to 18 out of 19. Six studies measured walking speed. The pooled improvement in walking speed was $0.13 \mathrm{~m} / \mathrm{s}(0.07-0.2)$ or $38 \%$ (22.18-53.8). PCI was also improved when the stimulator was used.

In conclusion, FES seems to have a positive orthotic effect on walking, also when compared with the conventional treatment. The type of stimulator (i.e. transcutaneous or implanted) seems not to influence the walking speed.
\end{abstract}




\section{Introduction}

Stroke is a major illness in Western countries with huge disabling consequences. The incidence of stroke in The Netherlands is approximately 30,000 per year and the prevalence is 120,000 patients (1). A stroke causes impairment of the cognitive, sensory, perceptive and motor functions. A rather common motor impairment is a dropped foot, which is characterised by the inability to dorsiflex the ankle, leading to insufficient toe clearance during walking. This impairment, in combination with commonly seen low selectivity of hip and knee in these patient group results in an abnormal gait, consisting of hip hitching, circumduction and toe catch, also called equine gait ${ }^{2}$. Walking speed is impaired and there is a higher chance of stumbling and falling.

An estimated $20 \%$ of the population with partial recovery have a drop foot (2). Out of 120,000 stroke survivors $75 \%$ recovers only partially, which are 90,000 patients. This group of 90,000 includes approximately 18,000 patients with a dropped foot.

The conventional treatment of drop foot is a splint, usually a custom fitted ankle-foot orthosis (AFO), which is a plastic support worn inside the shoe to maintain the ankle joint in a neutral position, and occasionally a more substantial splint attached to the shoe. This treatment has limitations, being both uncomfortable and awkward to use (3). In 1961, a new method for correction of drop foot by means of electrical stimulation was introduced by Liberson et al. (4). The stimulation was applied via electrodes on the skin and was synchronised with the gait phase by a heel-switch worn in the shoe. Stimulation was turned on when the heel was lifted at the beginning of the swing phase. It then produced dorsiflexion and eversion of the ankle joint. Stimulation was turned off when the heel was on the floor again.

A number of (theoretical) advantages of FES in comparison to an orthosis can be mentioned. The active contraction of the muscles stimulates the blood circulation, there is better afferent feedback, walking distance is increased, the stimulator is not custom made like an AFO and thus better applicable to a wide range of people and finally the stimulator is cosmetically better accepted (5). In addition, Merletti et al. mentions that walking with FES implies a more energy efficient use of the hip and knee muscles by avoiding the need for compensatory movements (6). However, the FES system is more sensitive to disturbance and the application requires more time, because of the placement of surface electrodes.

FES is not appropriate for all stroke patients with a dropped foot. The patient has to be well motivated, able to stand and walk either alone or with minimal assistance and the 
muscles that raise the foot should not be denervated. Contraindications are communication disorders, irritation of the skin and limited range of movement. The use of FES is not widespread and the total number of patients being treated remains quite small. This can be attributed to several reasons, such as technical limitations and unfamiliarity with FES in many countries. Technical limitations associated with the use of surface stimulators concern the lack of selectivity over the muscles and nerves recruited, the sensitivity of muscle recruitment to electrode placement and pain and tissue irritation associated with the passage of current through the skin (7).

In order to improve the selectivity upon stimulation responses, implantable systems are being developed (7-9). In contrast to the one-channel implantable stimulator, the twochannel stimulator provides separate control of the dorsiflexion and eversion movement by stimulating both deep and superficial peroneal nerves respectively. For more information about technical developments the reader is referred to a review of Lyons et al. (10). Preliminary trials have shown that it is possible to balance the foot well between inversion and eversion (8). The principle aim of implantable systems is to establish an orthotic effect rather than producing motor relearning effects. When motor relearning is the main goal, surface stimulators are more indicated.

Although the concept of FES of the n. peroneus exists for more than 40 years, there is no hard evidence for the positive clinical effects of this treatment.

Glanz et al. (11) performed a meta-analysis to assess the efficacy of FES on the force of the paretic muscles in the rehabilitation of stroke patients. They concluded that pooling from randomised trials supports FES as promoting recovery of muscle strength after stroke. A second review was carried out by Burridge et al. (12), who focussed on the orthotic and/or therapeutic effect of FES for the correction of dropped foot in subjects suffering from upper motor neuron lesions. However, their review had a descriptive character and study data were not pooled. Another aspect is that only surface stimulators to correct dropped foot were included. Their conclusion was that patients who benefit from FES, experience sufficient improvement in the speed and quality of walking to increase independence significantly.

The present systematic review was carried out to establish the available evidence of the orthotic effect of $n$. peroneus stimulation on walking speed. All types of stimulation approaches were included, i.e. surface, one- and two-channel implants. The orthotic effect is defined as the effect that occurs during stimulation while the therapeutic (carry-over) effect is the effect that remains even after the stimulator has been removed 
(12). The primary outcome measures selected from the present study are walking speed and Physiological Cost Index (PCI), which is a measure for energy cost. 


\section{Methods}

\section{Literature search}

A literature search was performed in PubMed, in the Database of Abstracts of Reviews of Effectiveness (DARE), Cochrane database, NHS Economic Evaluation Database (NHS EED), and the Health Technology Assessment Database (HTA) from the NHS centre for Reviews and Dissemination of the University of York. The PubMed database includes literature from 1966 up to 2003. The following keywords were used separately and combined in PubMed: cerebrovascular accident, electric stimulation, electric stimulation therapy, rehabilitation, recovery of function, peroneal nerve, muscle spasticity, walking, comparative study, cost-benefit analysis and evaluation study. In the other databases, the previous terms and the following additional terms were used: drop foot, dropped foot, ankle dorsiflexion, hemiplegia, FES, functional electric stimulation, peroneus and stroke.

Studies were included if they met the following criteria: 1 . functional electrical stimulation of the peroneal nerve should be applied to stroke patients with a dropped foot to improve walking, 2. transcutaneous or implantable stimulators should have been used, 3. comparative trial design, comparing FES with either another treatment or baseline status, 4. studies examining an orthotic effect or both an orthotic and therapeutic effect, 5. full-length articles in English or Dutch language published between 1966 up to 2003. To support our evidence we also looked for suitable proceedings of FES conferences that reported about the effect of peroneal nerve stimulation to improve walking. Since proceedings have not passed a peer-review process, it was decided not to include them into the pooled analysis. However, suitable proceedings will be described in the discussion section of the present review.

\section{Assessment of methodological quality}

As almost no randomised controlled trials with regard to peroneal nerve stimulation have been performed it was not possible to adopt the standard set of methodological criteria. Instead, we adapted the set in order to be able to summarise both controlled and uncontrolled trials. Eventually, a list of 16 criteria was used concerning patient selection, intervention, outcome measurement and statistics (Appendix 1). Two raters assessed the methodological quality of the included studies independently (AK, LO). In case of disagreement, consensus was reached by consulting a third rater (MY). All criteria that were answered with yes scored 1 point, with exception of criteria 3 , were 
the score varied from 1 to 3 points. When criteria $3 \mathrm{a}$, b or $\mathrm{c}$ was answered with yes they respectively scored 3, 2 and 1 point. By doing so, we accounted for the difference in methodological superiority of controlled vs. non-controlled trials. This because, from a methodological point of view, a randomised controlled trial is better than a cross-over design and a cross-over design is better than an observational study design. The maximum score that could be reached was 19. Since proceedings failed to meet the criteria that it should have been full-length publications, a methodological score was not determined for them.

\section{Data extraction}

Data was extracted from the articles and categorised using the following items: study design, patients, intervention, training, variables, measurements, statistics and miscellaneous. Then an inventory was made of the different outcome measures. A selection was made between clinical measures, i.e. walking speed, PCI and intermediate outcome measures, e.g. gait kinematics and spasticity.

\section{Data analysis}

Walking speed at a self-selected pace and PCI were considered to be the primary outcome measures. Walking speed changes in each of the articles were summarised and a pooled difference was estimated using 'random effects'-model (13). This statistical model is used in meta-analyses when both within-study sampling error (variance) and between-studies variation are included in the assessment of the uncertainty (confidence interval) of the results. Random effects models give wider confidence intervals than fixed effect models when there is significant heterogenity among the results of the included studies. 


\section{Results}

\section{Selection of literature}

The systematic literature search in PubMed resulted in the identification of 33 articles. The search in the other databases did not yield additional articles. Twenty-five studies were excluded from this review. Reasons of exclusion were that the study was not specifically about stroke patients (14-21), the study was not specifically about drop foot $(11,22-27)$, the study did not report on FES $(28,29)$ and the study was not a comparative trial (3,30-32). Two articles were about the same study and the second article did not yield additional information (33). The publications from Liberson, Buurke and Zilvold $(4,5,34)$ failed to meet the inclusion criteria that it should have been full-length publications written in Dutch or English between 1966 and 2003.

Eight studies fulfilled the selection criteria and were included in the present review (Table 2.1) (2,6-8,35-38). In addition, three proceedings from the IFESS conference (2000) were found about reporting the effect of peroneal nerve stimulation on walking speed in chronic stroke patients $(9,39,40)$.

\section{Characteristics of the included studies}

Patients. The number of patients included in the selected studies ranges from 2 to 56, with a total of 203 patients. In five studies chronic patients were included (2,7,8 36,38), in one study both chronic and sub-acute patients were included (6) and in two studies chronic, sub-acute and acute patients were included $(35,37)$. The first two weeks after the cerebrovasculair accident has been defined as the acute phase, the period between two weeks and 6 months after the accident as the sub-acute phase and the period after 6 months has been defined as the chronic phase. In total, 10 patients were in the acute stage, 17 patients in the sub-acute stage and 176 patients in the chronic stage after stroke.

Two of the studies were carried out in hospitalised patients $(35,38)$. Three patients dropped out in two studies each $(37,38)$. Of 203 patients, 101 were males, 44 were females and in 58 cases the gender was not mentioned. Paretic side was mentioned in six studies (2,6,35-38). Right hemiparesis was mentioned in 84 patients, left hemiparesis in 101 patients and in 18 cases the side was not mentioned. 
Study designs. Three different designs were used: one randomised controlled trial (RCT) (2), two cross-over studies $(35,37)$ and five times a within-subject comparison (6$8,36,38)$.

The method of FES varied between the studies. In five studies transcutaneous stimulation was used $(2,35-38)$ and in the other three studies implantable stimulation was applied (6-8).

In five studies, the patient could use the stimulator every day at home $(2,7,8,36,37)$. In three studies this was not the case. In the study of Stefanovska patients had a limit of 2 hours/day to use the stimulator, Bogataj used treatment sessions of 30min-1h 5 days a week and the patients in the study of Merletti used the stimulator for 1-4 hours, 5 days/week.

Outcome measures. In the eight included studies a total of 20 different outcome measures were used. Walking speed was measured in six studies $(2,7,8,35-37)$ and PCI was measured in two studies $(2,36)$. Merletti and Stefanovska both do not have walking speed or PCI as outcome parameter in their study.

In the present study, walking speed and PCI are considered to be the primary clinical endpoints. The other outcome measures like endurance, gait kinematics, gait kinetics, torque measurements, spasticity, EMG and $\mathrm{O}_{2}$ consumption are considered intermediate outcome measures.

\section{Methodological quality}

Scores for methodological quality ranged from 9 to 18 out of 19 (Table 2.2). There was a disagreement between both raters in $9.9 \%$ of the items. Consensus on these items was reached by a third rater. The RCT of Burridge (2) and the cross-over study by Granat (37) were methodologically the best articles with a score of both 18 points. Next, the cross-over study of Bogataj (35) reached the highest score, which scored 15 out of 19 points. 
Table 2.1 Characteristics of included studies

\begin{tabular}{|c|c|c|c|c|c|c|c|c|}
\hline Author & Waters (1975) & Merletti (1979) & Stefanovska (1988) & Bogataj (1995) & Granat (1996) & Burridge (1997a) & Burridge (1997b) & Kenney (2002) \\
\hline Study design & before/with & before/with & before/with & cross-over & cross-over & with and without FES & experimental (RCT) & before/with \\
\hline \multicolumn{9}{|l|}{ Patients } \\
\hline number (dropout) & 16 & $50(3)$ & 8 & 20 & $19(3)$ & 56 & 32 & $2(0)$ \\
\hline type & $12 / 16 \mathrm{CVA}$ & CVA & $5 / 8 \mathrm{CVA}$ & CVA & CVA & $50 / 56 \mathrm{CVA}$ & CVA & $\mathrm{CVA}$ \\
\hline age (sd) [range] & $49,3(11,3)$ & $57,3(12,9)$ & $45,4(5,9)$ & $56,3(10,4)$ & $57,8(9,4)$ & $54(12)$ & $56,8(16,6)$ & [31-48] \\
\hline stage after stroke & chronic & chronic & sub-acute+chronic & acute+sub-acute+chronic & acute+sub-acute+chronic & chronic & chronic & chronic \\
\hline gender & 8 male 8 female & 36 male 14 female & 7 male 1 female & 11 male 9 female & 16 male 3 female & not mentioned & 23 male 9 female & not mentioned \\
\hline paretic side & not mentioned & 14 right 36 left & 5 right 3 left & 9 right 11 left & 12 right, 7 left & 27 right 29 left 1 bi & 17 right 15 left & not mentioned \\
\hline \multicolumn{9}{|l|}{ Intervention } \\
\hline treatment & FES: implanted & FES: transcutaneous & FES: implanted & FES: transcutaneous & FES: transcutaneous & FES: transcutaneous & FES: transcutaneous & FES: implanted \\
\hline control & no control group & no control group & no control group & conventional therapy & physiotherapy & no control group & physiotherapy & no control group \\
\hline compared to & AFO & no aid & no aid & no aid & no aid & no aid & no aid & no aid \\
\hline \multicolumn{9}{|l|}{ Training } \\
\hline location & at home & hospital & at home & hospital & at home & at home & at home & at home \\
\hline duration & 6 months & 1-9 weeks & 6 months & 3 weeks & 4 weeks & 3 months & 12-13 weeks & 20 weeks \\
\hline intensity & daily, individually & 5 days/week, mean $14 \mathrm{~h}$ & 2 hours/day & $5 \mathrm{x} /$ week $30 \mathrm{~min}-1 \mathrm{~h}$ & daily, individually & daily, Individually & daily, individually & daily, individually \\
\hline \multicolumn{9}{|l|}{ Outcome measure } \\
\hline clinical endpoint & walking speed & & & walking speed & walking speed & walking speed, PCI & walking speed, PCI & walking speed \\
\hline other outcome & stride length & spasticity plantarflexors & max. isometr.torque: & $\begin{array}{c}\text { distance } \\
\text { number of strides, mean }\end{array}$ & heel strike, inversion & functional mobility & & endurance \\
\hline & cadence, EMG & $\begin{array}{c}\text { O2 consumption } \\
\text { Kendall scale: } \\
\text { dorsiflexion, eversion }\end{array}$ & $\begin{array}{l}\text { dorsal/plantarflexion } \\
\text { resistive torque } \\
\text { pas.mov. }\end{array}$ & $\begin{array}{c}\text { stride time } \\
\text { temporal symmetry, mean } \\
\text { stance time, Fugl-Meyer, } \\
\text { ground reaction forces }\end{array}$ & symmetry & & & isometric torque \\
\hline \multicolumn{9}{|l|}{ Measurements } \\
\hline moments & $\begin{array}{l}\text { every } 3 \mathrm{mo} \\
\text { with+without }\end{array}$ & every 2 weeks & $\begin{array}{c}\text { every } 6 \mathrm{mo} \\
\text { surf.stim. } 30 \mathrm{~min} / \mathrm{day}\end{array}$ & every 3 weeks & $0,6 \mathrm{wks}, 11 \mathrm{wks}$ & $6 \mathrm{wks}, 3 \mathrm{mo}$, every $6 \mathrm{mo}$ & $0,4-5 \mathrm{wks}, 12-13 \mathrm{wks}$ & $\begin{array}{c}0,32 \text { wks } \\
\text { with/without }\end{array}$ \\
\hline baseline & orthosis & without aids & $2-3 \mathrm{wks}$ & not mentioned & usual aids & with and without FES & usual aids+FES & usual aids \\
\hline follow-up & with+without FES & with+without FES & without FES & after MFES+conv therapy & with+without FES & with and without FES & $\begin{array}{l}\text { with+without FES } \\
\text { Wilcoxon, Mann- }\end{array}$ & with FES \\
\hline \multirow[t]{2}{*}{ statistics } & not mentioned & Student's t-test & not mentioned & MANOVA & ANOVA, paired t-test & paired t-test & Whitney & not mentioned \\
\hline & & & & Friedmans' ANOVA & Wilcoxon & Fisher's exact test & chi-square, Spearman & \\
\hline
\end{tabular}


Table 2.2 Overview of methodological scores of each of the articles. The scores are separated for each part of the list (maximum methodological score)

\begin{tabular}{|c|c|c|c|c|c|c|}
\hline Author & Total (19) & Part $1^{1}(5)$ & Part $2^{2}(4)$ & Part $3^{3}(6)$ & Part $4^{4}(4)$ & Disagreement \\
\hline Waters (1975) & 12 & 2 & 3 & 4 & 3 & 2 \\
\hline Merletti (1979) & 14 & 3 & 3 & 4 & 4 & 1 \\
\hline Stefanovska (1988) & 9 & 1 & 3 & 4 & 1 & 2 \\
\hline Bogataj (1995) & 15 & 4 & 3 & 4 & 4 & 1 \\
\hline Granat (1996) & 18 & 5 & 3 & 6 & 4 & 2 \\
\hline Burridge I (1997a) & 13 & 3 & 1 & 6 & 3 & 2 \\
\hline Burridge II (1997b) & 18 & 5 & 3 & 6 & 4 & 2 \\
\hline Kenney (2002) & 9 & 1 & 2 & 5 & 1 & 3 \\
\hline \multicolumn{7}{|l|}{${ }^{1}$ patient selection } \\
\hline \multicolumn{7}{|l|}{${ }^{2}$ intervention } \\
\hline \multicolumn{7}{|l|}{${ }^{3}$ outcome measures } \\
\hline${ }^{4}$ statistics & & & & & & \\
\hline
\end{tabular}

\section{Effect of functional electrical stimulation on walking speed}

Table 2.3 shows the measured walking speeds with and without FES and the difference between both measurements. Six of the eight studies measured comfortable walking speed $(2,7,8,35-37)$. It was not possible to calculate differences in walking speed for all studies due to insufficient data presentation $(8,36)$. Correspondence with the authors failed to provide the missing data.

In both studies performed by Burridge $(2,36)$ the 10 -meter walking test was used to measure walking speed. One meter was allowed at the start and finish of the walkway for acceleration and deceleration. In the study of Granat (37) the length of the recorded walk path was either 6 or 10 meters, dependent on the ability of the participating patient. They used a 1.5 meter lead-in and run-out of the test walk path in stead of 1 meter. Also in the study of Kenney ${ }^{8}$ the 6-meter test was used in both included patients. Bogataj (35) measured walking speed over a distance of 20 meters and Waters (7) did not mention how they measured walking speed. All studies measured walking speed three times, with exception of the study of Granat (37), who measured walking speed five times. Waters (7) again did not give information about this.

In three studies, a significant improvement in walking speed was found $(2,7,35)$. Two other studies only reported the percentage of difference without providing a measure of variability $(8,36)$ and the last study did not show a significant change (37). This study, performed by Granat and colleagues, was the only study who found a small decrease in walking speed after the treatment period, from 0.94 to $0.93 \mathrm{~m} / \mathrm{s}$. 
The pooled improvement in walking speed was $0.13 \mathrm{~m} / \mathrm{s}(0.07-0.2)$ or $38 \%(22.18-53.8)$.

Table 2.3 Walking speed

\begin{tabular}{|c|c|c|c|c|c|c|c|}
\hline Author & $\mathbf{N}$ & $\begin{array}{l}\text { Meth. } \\
\text { quality }\end{array}$ & Stimulator & $\begin{array}{c}\text { Before }(\mathrm{m} / \mathrm{s}) \\
\text { mean }(\mathrm{sd})\end{array}$ & $\begin{array}{c}\text { After }(\mathrm{m} / \mathrm{s}) \\
\text { mean }(\mathrm{sd})\end{array}$ & $\begin{array}{c}\text { Difference } \\
(\mathrm{m} / \mathrm{s}) \\
\text { mean }(\mathrm{sd})\end{array}$ & $\begin{array}{l}\text { Difference (\%) } \\
\text { mean }(95 \% \mathrm{CI})\end{array}$ \\
\hline $\begin{array}{l}\text { Waters } \\
(1975)^{1}\end{array}$ & 16 & 12 & Implanted & $\begin{array}{c}0.58 \\
(0.25)\end{array}$ & $\begin{array}{c}0.79 \\
(0.26)\end{array}$ & $\begin{array}{l}0.21 \\
(0.1)\end{array}$ & $\begin{array}{c}36 \\
(16.7-55.7)\end{array}$ \\
\hline $\begin{array}{l}\text { Bogataj } \\
(1995)^{2} \\
\text { C }\end{array}$ & 10 & 15 & Transcut. & $\begin{array}{c}0.23 \\
(0.13)\end{array}$ & $\begin{array}{c}0.26 \\
(0.11)\end{array}$ & $\begin{array}{c}0.20 \\
(0.07)\end{array}$ & $\begin{array}{c}104 \\
(59.4-149.2)\end{array}$ \\
\hline I & 10 & & & $\begin{array}{c}0.19 \\
(0.09)\end{array}$ & $\begin{array}{c}0.41 \\
(0.21)\end{array}$ & & \\
\hline $\begin{array}{l}\text { Granat } \\
(1996)^{3}\end{array}$ & 16 & 18 & Transcut. & $\begin{array}{c}0.94 \\
(0.63)\end{array}$ & $\begin{array}{c}0.93 \\
(0.59)\end{array}$ & $\begin{array}{l}-0.01 \\
(0.21)\end{array}$ & $\begin{array}{c}-0.8 \\
(-46.2-44.6)\end{array}$ \\
\hline $\begin{array}{l}\text { Burridge I } \\
(1997 a)^{4}\end{array}$ & 56 & 13 & Transcut. & - & - & - & 14 \\
\hline $\begin{array}{l}\text { Burridge II } \\
(1997 b)^{5} \\
\text { C }\end{array}$ & 16 & 18 & Transcut. & $\begin{array}{c}0.48 \\
(0.25)\end{array}$ & $\begin{array}{c}0.51 \\
(0.27)\end{array}$ & $\begin{array}{c}0.1 \\
(0.04)\end{array}$ & $\begin{array}{c}14 \\
(-36.4-64.5)\end{array}$ \\
\hline I & 16 & & & $\begin{array}{c}0.64 \\
(0.46)\end{array}$ & $\begin{array}{c}0.77 \\
(0.43)\end{array}$ & & \\
\hline $\begin{array}{l}\text { Kenney } \\
(2002)^{6} \\
\end{array}$ & 2 & 9 & Implanted & - & - & - & 27 \\
\hline
\end{tabular}

1 before with orthosis, after with stimulation

2 difference between assessment 1 (baseline) and 2 (C: conv. therapy, I: conv. therapy and FES)

3 linoleum surface, session 2 is used as before, session 3 with PS as after

4 after 3 months, difference between with and without stimulation

5 before FES group without stimulation, after FES group with stimulation

${ }^{6}$ mean of two subjects

$\mathrm{C}=$ control group

$\mathrm{I}=$ intervention group

Figure 2.1 shows the effect of the stimulator on walking speed for each of the articles with its mean and the $95 \%$ confidence interval. The methodological score was included between brackets. There seems to be no clear relation between methodological quality of the studies and the reported effect of functional electrical stimulation. 


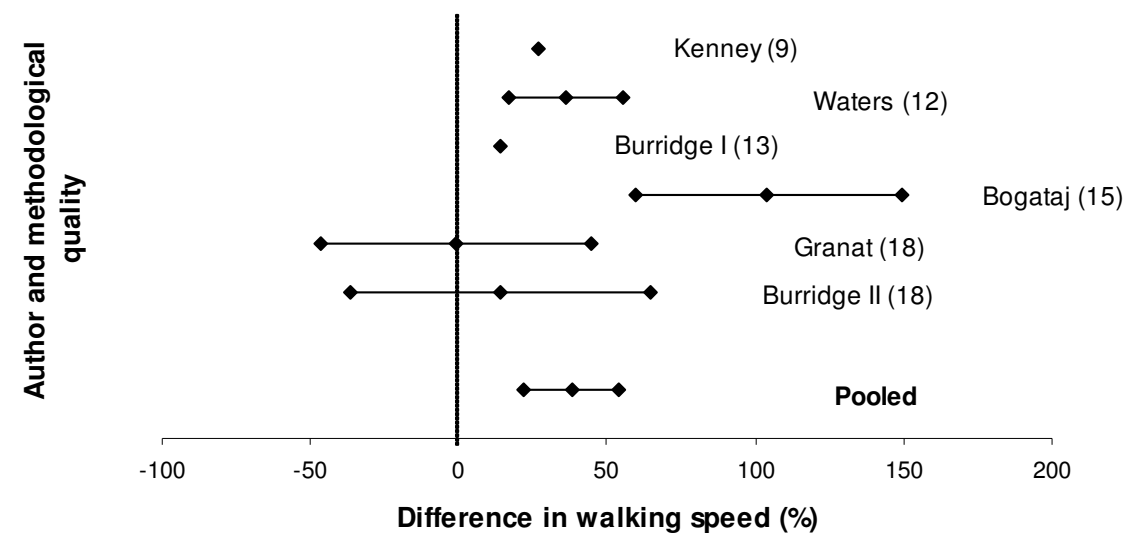

Figure 2.1 Methodological quality and difference in walking speed (\%)

Notes: Mean and $95 \%$ confidence interval is presented

\section{Effect of functional electrical stimulation on Physiological Cost Index (PCI)}

Two of eight studies, both carried out by Burridge, measured PCI $(2,36)$. The first study showed a decrease of $39.5 \%$ in PCI, comparing PCI with and without stimulation after three months. There was no significant change in PCI over 3 months either with or without the stimulator. The second study, which was a RCT, showed an improvement of $24.9 \%$ in the FES group when the stimulator was used, in a period of $12-13$ weeks. Improvement was also measured in the control group with a reduction of $1 \%$ in PCI. 


\section{Discussion}

In the present review, the results of eight studies were analysed in order to assess the orthotic effect of FES on the improvement of walking in stroke patients with a dropped foot. Six of the eight studies measured walking speed and five of them suggest a positive effect of FES on walking. These studies, with exception of the study performed by Waters, made a comparison between walking with and without stimulation. Waters and associates made a comparison between walking speed preoperative with an orthosis and walking speed after surgery with stimulation. They found that walking speed was increased significantly (36\%) by stimulation (7).

In conclusion, FES seems to have a positive orthotic effect on walking, also when compared with the conventional treatment. The type of stimulator (i.e. transcutaneous or implanted) seems not to influence the walking speed.

Also the proceedings, which were not included in the pooled analyses, showed a positive effect of peroneal nerve stimulation on walking speed. Haugland and colleagues (9) found that the orthotic effect on walking speed seen with an external stimulator was variable, probably depending on the exact placement of the electrodes, but for an implanted stimulator was almost constant and at the level of the strongest effect obtained with the external stimulator. No therapeutic effect was found.

In a study performed by Matsunaga et al. (39), all six patients were able to walk faster and longer when using the stimulator system. Their patients showed a mean improvement of $14.8 \%$ in walking speed.

Mann and colleagues (40) also measured the effect of peroneal nerve stimulation, but then in combination with stimulating a second channel. Selection of the second muscle group was based on clinical observation. Their results indicate a significant therapeutic and orthotic effect on walking speed from using a second channel of stimulation, greater than that achieved with single channel stimulation alone.

\section{Choice of patients}

Only 6 of 203 patients dropped out, which is quite remarkable. Two studies described that almost all patients continued to use the stimulator after the trial had ended $(2,36)$. These findings could indicate that the use of the stimulator is not too difficult and patients are satisfied with the effects. Another explanation might be that the selection procedure of patients was successful. It is well known from the literature that 'FES' is a useful orthotic device for a selected sub-population of hemiplegic patients. According to 
Merletti and colleagues (6) and Gracanin (41), about $20 \%$ of the ambulant hemiplegic population benefits from common peroneal nerve stimulation during the rehabilitation period. Granat (37) concludes that the stimulator applied in the late stage of rehabilitation would be applicable to a few patients (2\%), particularly in patients with medio-lateral instability of the foot and reduced ground clearance in swing leading to forefoot contact. According to Burridge (2) and Waters (7) the stimulator does not work for everyone, although it was not mentioned to which criteria a patient should fulfil to be suitable. Carnstam et al. (14) found that careful selection led to a $94 \%$ success rate. In conclusion, there seems to be no consensus about subgroup specific effects of peroneal nerve stimulation. The idea is that people with higher initial walking speed perform better than people with lower initial walking speed. However, this could not be confirmed in the present study, because individual walking speed data was not mentioned in the included studies. Only the study performed by Bogataj and associates (35) reported the individual walking speed data of all participants. These data showed no distinct relation between the initial walking speed of patients and their improvement after the use of FES.

\section{Included studies}

Unfortunately, literature justifying the use of stimulation to correct dropped foot is mainly based on case studies, uncontrolled trials and retrospective reviews. In the present review only one RCT was included (2), which is obviously the most reliable design to separate specific from non-specific effects. They have become the gold standard for the evaluation of treatment efficacy (42-44). Five of the eight included studies were open label studies, which means that there was no control group (6$8,36,38)$. The two remaining studies were cross-over studies $(35,37)$, which design could be a problem in comparative trials using FES, because of a possible carry-over effect (45).

For the present review it was assumed that, although non-randomised studies have methodological problems, they could actually produce effect sizes as generated in randomised studies. In this review, most of the patients (176/203) were in the chronic stage after stroke. The chance of spontaneous recovery in these patients is negligible so an observed effect can not easily be attributed to this. Therefore correction for natural recovery by randomisation seems not essential. Two studies measured not only chronic stroke patients, but also acute and subacute patients $(35,37)$. Remarkable is the 
difference in walking speed measured at baseline between both studies. The difference between Bogataj et al. (35) and Granat et al. (37) is $0.73 \mathrm{~m} / \mathrm{s}$ or 3.5 times faster. Bogataj et al. did not mention details about baseline measurements so the difference can not be explained by baseline measurement or selection procedure.

The pooled analysis of both controlled and uncontrolled trials showed an improvement of 38\% in walking speed with a confidence interval of $22.18-53.8 \%$.

Perry et al. (46) made a classification of walking handicap in the stroke population. They described that the least limited community walkers should walk with a mean velocity of $0.58 \mathrm{~m} / \mathrm{s}$. Community walkers should walk with a mean velocity of $0.8 \mathrm{~m} / \mathrm{s}$. So an improvement of $0.22 \mathrm{~m} / \mathrm{s}$ is clinically relevant according to Perry. Stroke patients who normally use a peroneus stimulator, especially those who use an implantable stimulator, are relatively good patients who are not very limited in their daily activities. The studies performed by Waters et al. (7) and Bogataj et al. (35) managed to approach this size of improvement. They measured a mean improvement of respectively $0.21 \mathrm{~m} / \mathrm{s}$ and $0.20 \mathrm{~m} / \mathrm{s}$.

An alternative way of looking at the results is to consider the percentage change. Burridge et al. (2) decided that a $10 \%$ improvement in walking speed was considered to be functionally relevant. In the present study, this improvement is reached by all studies, with exception of the study performed by Granat et al. (37), who measured a worsening in walking speed of $-0.8 \%$.

\section{Orthotic versus therapeutic benefit}

The present review was only focussed on the orthotic effect of FES on walking speed in stroke patients with a dropped foot.

Another interesting aspect to investigate is the possible therapeutic or carry-over effect of FES, which can be defined as the benefit gained following a period of stimulation. Liberson and associates noted that when footdrop was corrected in hemiplegic patients by means of electrical stimulation using cutaneous electrodes, some retained the ability to dorsiflex for varying lengths of time after stimulation was stopped (4). Waters et al. (7) observed the same phenomenon in some of their patients. They found an improvement in gait velocity without stimulation, compared with the velocity without an orthosis before surgery. The testing of these patients took place immediately after walking with stimulation. The review of Burridge et al. (12) also concluded that some studies $(14,47,48)$ reported a carry-over effect, which consisted of increased voluntary 
movement and reduced spasticity, but that it is unclear how this occurs, whether this effect is permanent, or how suitable patients can be identified. Many of the included studies were with small samples and few used convincing methodology.

Overall, the literature shows no convincing therapeutic effect of peroneal nerve stimulation. When realisation of a therapeutic effect is the main goal, which might especially be the case in acute stroke patients, surface stimulators are more indicated than implantable stimulators. Surface stimulators are therefore useful devices for gait training in acute patients at rehabilitation centres. Due to the difficulties involved in a proper electrode placement, training in the home situation is often cumbersome in the beginning. Good instructions of a care professional are needed for this. An advantage of using the stimulator also at home is that therapy duration is not limited by time, so that patients can practice as much as they want. The prescription of an implantable peroneal nerve stimulator is only a treatment option when the main goal is to realise an orthotic effect for the long term in drop foot patients. In these patients an implantable stimulator offers greater comfort when compared to a surface stimulator.

\section{Conventional treatment}

In the present review, only three of the eight studies included a control group $(2,35,37)$. The control group in the study of Burridge (2) and Granat (37) both received physiotherapy. As only Granat described that during the control period the patients received their normal physiotherapy, it was not possible to examine if there was a difference in treatment intensity between both studies. The conventional treatment in the study of Bogataj (35) was much more comprehensive, consisting of physical therapy, medical treatment, occupational therapy, speech therapy, sessions with a psychologist, sessions with a social worker and a cultural program. These studies show that different conventional treatments exist with a large variation in intensity to treat stroke patients with a dropped foot, which makes it difficult to compare their results.

Dropped foot is conventionally corrected by splinting. As far as we know, only one (placebo-controlled) randomised clinical trial (49) is performed to examine the effect of an $\mathrm{AFO}$ on the walking ability in stroke patients. Beckerman et al. included 60 patients and they combined treatment with a polypropylene AFO, thermocoagulation (TH), placebo-AFO and placebo-TH treatment, which resulted in four groups. The results show that the efficacy of both therapeutic interventions appears to be neither statistically significant nor clinically relevant. Only in the AFO group there was a small, 
but clinically irrelevant increase of $0.1 \mathrm{~m} / \mathrm{s}$ in comfortable and maximum walking speed in comparison with the placebo-AFO group. Also 2/3 of all included patients were unsatisfied with the use of the AFO, which was measured with the Sickness Impact Profile.

An interesting aspect that has not been clarified yet is the additional value of the peroneus stimulator in comparison with an AFO. Numerous (observational) studies have reported the effect of using an AFO or FES separately, but Mann et al. (50) has made a comparison between both treatments. In this study chronic stroke patients were randomly assigned to use either an AFO or a surface stimulator for 12 weeks to manage their dropped foot. Significant improvements in walking speed, endurance and mobility were observed after 12 weeks in both groups. The FES group showed a significant carryover effect in their unaided walking over 12 weeks, which was not observed in the AFO group. Also a larger trend towards improved PCI was observed in the FES group compared to the AFO group. These results support the hypothesis that FES may have a greater training effect than simply using an AFO to correct a dropped foot in chronic stroke patients. Further work is however required to investigate this more comprehensively.

\section{Conclusion}

FES seems to have a positive orthotic effect on walking speed and PCI. The pooled effect size for walking speed was $0.13 \mathrm{~m} / \mathrm{s}(0.07-0.2)$ or $38 \%$ (22.18-53.8).

Walking speed seems also to increase when FES is compared with an AFO. In the literature it is not clear what proportion might benefit from FES. Future studies should report about suitability criteria for patients.

PCI is found to decrease in two studies $(2,36)$. In one study, there was a significant decrease in PCI with and without stimulation after three months (36). No significant changes were found when comparing PCI before and after treatment. Although patients often reported that walking was less fatiguing this seemed to be a psychological effect. 


\section{References}

1. Publication of the Dutch Heart Foundation (in Dutch: Nederlandse Hartstichting); viewed on:08-07-2002; http://www.hartstichting.nl/static/prof_hoofd/profhfd_factsheet_beroerte. htm.

2. Burridge JH, Taylor PN, Hagan SA, Wood DE, Swain ID. The effects of common peroneal stimulation on the effort and speed of walking: a randomized controlled trial with chronic hemiplegic patients. Clin Rehabil 1997(b) Aug;11(3):201-10.

3. Taylor PN, Burridge JH, Dunkerley AL, Lamb A, Wood DE, Norton JA, Swain ID. Patients' perceptions of the Odstock Dropped Foot Stimulator (ODFS). Clin Rehabil. 1999 Oct;13(5):439-46.

4. Liberson WT, Holmquest ME, Scot D, Dow M. Functional electrotherapy: stimulation of the peroneal nerve synchronized with the swing phase of the gait of hemiplegic patients. Arch Phys Med Rehabil 1961;42:101-5.

5. Buurke JH, Schlecht M, Bouwman R. Ist der Peronaeus -Stimulator eine sinnvolle Alternative zur Unterschenkelorthese? -Technik, Anwendung und Ergebnisse. Med. Orth. Tech. 110;1990:60-3.

6. Merletti R, Andina A, Galante M, Furlan I. Clinical experience of electronic peroneal stimulators in 50 hemiparetic patients. Scand J Rehabil Med 1979;11(3):111-21.

7. Waters RL, McNeal D, Perry J. Experimental correction of footdrop by electrical stimulation of the peroneal nerve. J Bone Joint Surg Am 1975 Dec;57(8):1047-54.

8. Kenney L, Bultstra G, Buschman R, Taylor P, Mann G, Hermens H, Holsheimer J, Nene A, Tenniglo M, van der Aa H, Hobby J. An implantable two channel drop foot stimulator: initial clinical results. Artif Organs 2002 Mar;26(3):267-70.

9. Haugland, M, Childs C, Ladouceur M, Haase J and Sinkjaer T: An implantable foot drop stimulator. Proc. $5^{\text {th }}$ annual conference of IFESS, Aalborg, Denmark 2000:59-62.

10. Lyons GM, Sinkjær T, Burridge JH, Wilcox DJ. A review of portable FES-based neural orthoses for the correction of drop foot. IEEE transactions on neural systems and rehabilitation engineering 2002;10(4):260-79.

11. Glanz M, Klawansky S, Stason W, Berkey C, Chalmers TC. Functional electrostimulation in poststroke rehabilitation: a meta-analysis of the randomized controlled trials. Arch Phys Med Rehabil 1996 Jun;77(6):549-53.

12. Burridge JH, Swain ID, Taylor PN. Functional electrical stimulation: a review of the literature published on common peroneal nerve stimulation for the correction of dropped foot. Reviews in Clinical Gerontology 1998;8:155-61.

13. DerSimonian R, Laird NM. Meta-analysis in clinical trials. Control Clin Trials 1986;7:17788 . 
14. Carnstam B, Larsson LE, Prevec TS. Improvement of gait following functional electrical stimulation. I. Investigations on changes in voluntary strength and proprioceptive reflexes. Scand J Rehabil Med. 1977;9(1):7-13.

15. Kljajic M, Malezic M, Acimovic R, Vavken E, Stanic U, Pangrsic B, Rozman J. Gait evaluation in hemiparetic patients using subcutaneous peroneal electrical stimulation. Scand J Rehabil Med 1992 Sep;24(3):121-6.

16. Stefanovska A, Vodovnik L, Gros N, Rebersek S, Acimovic-Janezic R. FES and spasticity. IEEE Trans Biomed Eng. 1989 Jul;36(7):738-45.

17. Tabeke K, Basmaijan JV. Gait analysis in stroke patients to assess treatments of foot-drop. Arch Phys Med Rehabil 1976 Jul;57(1):305-10.

18. Tabeke K, Kukulka C, Narayan MG, Milner M, Basmaijan JV. Peroneal nerve stimulator in rehabilitation of hemiplegic patients. Arch Phys Med Rehabil 1975 Jun;56(6):237-9.

19. Taylor PN, Burridge JH, Dunkerley AL, Wood DE, Norton JA, Singleton C, Swain ID. Clinical use of the Odstock Dropped Foot Stimulator: its effect on the speed and effort of walking. Arch Phys Med Rehabil. 1999 Dec;80(12):1577-83.

20. Taylor P, Burridge J, Dunkerley A, Wood D, Norton J, Singleton C, Swain I. Clinical audit of 5 years provision of the Odstock Dropped Foot Stimulator. Artif Organs 1999 May;23(5):440-2.

21. Voigt M, Sinkjaer T. Kinematic and kinetic analysis of the walking pattern in hemiplegic patients with foot-drop using a peroneal nerve stimulator. Clin Biomech (Bristol, Avon) 2000 Jun;15(5):340-51.

22. Bogataj U, Gros N, Malezic M, Kelih B, Kljajic M, Acimovic R. Restoration of gait during two to three weeks of therapy with multichannel electrical stimulation. Phys Ther. 1989 May;69(5):319-27.

23. Daly JJ, Ruff RL, Haycook K, Strasshofer B, Marsolais EB, Dobos L. Feasibility of gait training for acute stroke patients using FNS with implanted electrodes. J Neurol Sci. 2000 Oct 1;179(S 1-2):103-7.

24. Daly JJ, Ruff RL. Electrically induced recovery of gait components for older patients with chronic stroke. Am J Phys Med Rehabil. 2000 Jul-Aug;79(4):349-60.

25. Malezic M, Bogataj U, Gros N, Kelih B, Kljajic M, Acimovic-Janezic R. Evaluation of gait with multichannel electrical stimulation. Orthopedics 1987 May;10(5):769-72.

26. Malezic M, Bogataj U, Gros N, Decman I, Vrtacnik P, Kljajic M, Acimovic-Janezic R. Application of a programmable dual-channel adaptive electrical stimulation system for the control and analysis of gait. J Rehabil Res Dev 1992 Fall;29(4):41-53.

27. Strojnik P, Acimovic R, Vavken E, Simic V, Stanic U. Treatment of drop foot using an implantable peroneal underknee stimulator. Scand J Rehabil Med 1987;19(1):37-43. 
28. Levin MF, Hui-Chan CW. Relief of hemiparetic spasticity by TENS is associated with improvement in reflex and voluntary motor functions. Electroencephalogr Clin Neurophysio 1992 Apr;85(2):131-42.

29. Veltink PH, Ladouceur M, Sinkjaer T. Inhibition of the triceps surae stretch reflex by stimulation of the deep peroneal nerve in persons with spastic stroke. Arch Phys Med Rehabil. 2000 Aug;81(8):1016-24.

30. Burridge JH, McLellan DL. Relation between abnormal patterns of muscle activation and response to common peroneal nerve stimulation in hemiplegia. J Neurol Neurosurg Psychiatry 2000 Sep;69(3):353-61.

31. Kumar VP, Lau HK, Liu J, Pereira BP, Pho RW. Clinical applications of functional electrical stimulation. Ann Acad Med Singapore 1995 May;24(3):428-35. Review.

32. Rozman J, Acimovic-Janezic R, Tekavcic I, Kljajic M, Trlep M. Implantable stimulator for selective stimulation of the common peroneal nerve: a preliminary report. J Med Eng Technol 1994 Mar-Apr;18(2):47-53.

33. Waters RL, McNeal DR, Faloon W, Clifford B. Functional electrical stimulation of the peroneal nerve for hemiplegia. Long-term clinical follow-up. J Bone Joint Surg Am 1985 Jun;67(5):792-3.

34. Zilvold G. Functionele electrostimulatie van de nervus peroneus. Meppel: Krips Repro, 1976.

35. Bogataj U, Gros N, Kljajic M, Acimovic R, Malezic M. The rehabilitation of gait in patients with hemiplegia: a comparison between conventional therapy and multichannel functional stimulation therapy. Phys Ther. 1995 Jun;75(6):490-502.

36. Burridge J, Taylor P, Hagan S, Swain I. Experience of clinical use of the Odstock Dropped Foot Stimulator. Artif Organs 1997(a) Mar;21(3):254-60.

37. Granat MH, Maxwell DJ, Ferguson AC, Lees KR, Barbenel JC. Peroneal stimulator; evaluation for the correction of spastic drop foot in hemiplegia. Arch Phys Med Rehabil. 1996 Jan;77(1):19-24.

38. Stefanovska A, Gros N, Vodovnik L, Rebersek S, Acimovic-Janezic R. Chronic electrical stimulation for the modification of spasticity in hemiplegic patients. Scand J Rehabil Med Suppl. 1988;17:115-21.

39. Matsunaga T, Shimada Y, Sato M, Hatakeyama K, Chida S, Sato K, Misawa A, Ando S, Minato T, Konishi N, Iizuka K. The akita heel sensor system (AHSS) for the correction of dropped foot gait in hemiplegic patients. Proc. $5^{\text {th }}$ annual conference of IFESS, Aalborg, Denmark 2000:394-5.

40. Mann GE, Burridge JH, Ewins DJ, McLellan DL, Swain ID, Taylor PN, Wood DE, Wright PA. Optimising two channel stimulation to improve walking following stroke. Proc. $5^{\text {th }}$ annual conference of IFESS, Aalborg, Denmark 2000:452-5. 
41. Vodovnik L, Bajd T, Kralj A, Gracanin F, Strojnik P. Functional electrical stimulation for control of locomotor systems. CRC Critical Rev. Bioeng 1981;6(2):63-131.

42. Byar DP, Simon RM, Friedewald WT, Schlesselman JJ, DeMets DL, Ellenberg JH, Gail $\mathrm{MH}$, Ware JH. Randomized clinical trials: perspectives on some recent ideas. N Engl J Med 1976;295:74-80.

43. Feinstein AR. Current problems and future challenges in randomized clinical trials. Circulation 1984;70:767-74.

44. Abel U, Koch A. The role of randomization in clinical studies: myths and beliefs. J Clin Epidemiol 1999;52:487-97.

45. IJzerman MJ, Baardman G, Hermens HJ, Veltink PH, Boom HBK, Zilvold G. Comparative trials on hybrid walking systems for people with paraplegia: an analysis of study methodology. Prosthetics and Orthotics International 1999;23:260-73.

46. Perry J, Garrett M, Gronley JK, Mulroy SJ. Classification of walking handicap in the stroke population. Stroke 1995;26:982-9.

47. Stefancic M, Rebersek M, Merletti R. The therapeutic effects of the Ljubljana functional electronic brace. Eur Medicophys 1976;12:1-9.

48. Merletti R, Zelaschi F, Latella D, Galli M, Angeli S, Sessa MB. A control study of muscle force recovery in hemiparetic patients during treatment with functional electrical stimulation. Scand J Rehab Med 1978;10(3):147-54.

49. Beckerman H, Becher J, Lankhorst GJ, Verbeek AL. Walking ability of stroke patients: efficacy of tibial nerve blocking and a polypropylene ankle-foot orthosis. Arch Phys Med Rehabil 1996;77(11):1144-51.

50. Mann GE, Wright PA, Swain ID. Training effects of electrical stimulation and the conventional ankle foot orthosis in the correction of drop foot following stroke. FESnet 2002 Abstract submission 2000. 
Appendix I: Criteria list for assessment of methodological quality

\section{Patient selection}

1. Were the eligibility criteria specified?

Yes/No/Don't know

2. Was the selection procedure described?

Yes/No/Don't know

3. Was the design of the study:
a) a randomised controlled trial?
Yes: score 3
| No
b) a cross-over?
Yes: score 2
| No
c) open label?
Yes: score 1

\section{Intervention}

4. Was the index intervention explicitly described?

Yes/No/Don't know

5. Were co-interventions avoided of comparable?

Yes/No/Don't know

6. Was the compliance acceptable in all groups?

Yes/No/Don't know

7. Was baseline described?

Yes/No/Don't know

\section{Outcome measurements}

8. Were the outcome measures relevant?

Yes/No/Don't know

9. Were adverse effects described?

Yes/No/Don't know

10. Was the withdrawal/dropout rate described and acceptable?

11. 1) Was a short-term follow-up measurement $(\leq 2 \mathrm{mnd})$ described?

Yes/No/Don't know

Yes/No/Don't know

2) Was a long-term follow-up measurement ( $\geq 2 \mathrm{mnd}$ ) described?

Yes/No/Don't know

12. Were outcomes standardised?

Yes/No/Don't know

\section{Statistics}

13. Was the sample size for each group described?

Yes/No/Don't know

14. Is the number of patients at least 15 ?

Yes/No/Don't know

15. Were point estimates and measures of variability presented for the primary outcome measures?

Yes/No/Don't know

16. Were statistical tests performed and described? Yes/No/Don't know 

Chapter 3

A randomized controlled trial of an implantable two-channel peroneal nerve stimulator on walking speed and activity in post-stroke hemiplegia

Anke I.R. Kottink

Hermie J. Hermens

Anand V. Nene

Martin J.B. Tenniglo

Hans E. van der Aa

Hendrik P.J. Buschman

Maarten J. IJzerman

Arch Phys Med Rehabil 2007;88:971-8.

Reprinted with permission 


\section{Abstract}

The objective was to determine the effect of a new implantable two-channel peroneal nerve stimulator on walking speed and daily activities, in comparison with the usual treatment in chronic stroke survivors with a drop foot.

Twenty-nine stroke survivors with chronic hemiplegia with drop foot fulfilled the predefined inclusion and exclusion criteria and were included in the present randomized controlled trial. By means of block randomization patients were classified in the intervention and control group. All subjects were measured 5 times in the gait laboratory. The intervention group received an implantable two-channel peroneal nerve stimulator for correction of their drop foot. The control group continued using their conventional walking device, consisting of an ankle-foot orthosis, orthopedic shoes, or no device.

Walking speed, assessed both by a six-minute walk test (6MWT) and by using a 10-m walkway, was selected as primary outcome measure and activity monitoring data, consisting of percentage time spent on stepping, standing, and sitting/lying were selected as secondary outcome measure.

Functional electric stimulation resulted in a $23 \%$ improvement of walking speed measured with the 6MWT, whereas the improvement in the control group was only 3\% $(\mathrm{p}=.010)$. Comfortable walking speed measured on a $10-\mathrm{m}$ walkway was also significantly improved in favor of FES ( $\mathrm{p}=.038)$. The percentage time spent on stepping deteriorated with $3 \%$ in the intervention and $0.8 \%$ in control group, which was not statistically significant between both groups $(\mathrm{p}=.13)$.

The results of this study indicated a clinically relevant effect of the implantable twochannel peroneal nerve stimulator on walking speed in the sample of stroke survivors included in our study. 


\section{Introduction}

Foot drop or drop foot is a simple term that describes a rather complex problem. A variety of conditions, such as dorsiflexor injuries, peripheral nerve injuries, stroke, neuropathies, drug toxicities, or diabetes can be associated with drop foot. Depending on the cause, drop foot may be temporary or permanent. It can be defined as a significant weakness or absence of ankle and toe dorsiflexors. These muscles assist in clearing the foot during swing phase and control plantarflexion of the foot on heel strike. Weakness or absence of this group of muscles associated with imbalance between invertors and evertors results in an equinovarus deformity. Walking becomes a challenge due to the patient's inability to control the foot at the ankle. In fact, due to predominance of extensor synergy, hip and knee flexion are usually both reduced which further lengthens the limb functionally. Accordingly, many stroke survivors use circumduction and hip hiking and on occasion vaulting in order to compensate. This article focuses on drop foot in subjects with chronic hemiplegia after stroke. Drop foot after stroke is thought to be caused partly by poor active control of the anterior tibial muscle and by increased and inappropriate tone in the muscles of the leg, particularly the calf (1).

Functional electric stimulation (FES) is the clinical application of electric current to the intact nerves of the body, in order to generate a muscle contraction. This contraction is then incorporated into a functional activity, for example, walking. FES systems for the treatment of drop foot are in clinical use in significant numbers, especially the surface Odstock Drop Foot Stimulator (2-4). These studies showed an increased walking speed and a reduction in Physiological Cost Index (PCI), which is a measure for energy cost. The perception of the users was that the Odstock stimulator was of clinical benefit and therefore the compliance was very high.

In a systematic review (5), the results of 8 studies were analyzed to assess the orthotic effect of FES on walking in stroke survivors with a drop foot. The pooled effect size for walking speed was $.13 \mathrm{~m} / \mathrm{s}$ (range, $.07-0.2 \mathrm{~m} / \mathrm{s}$ ) or $38 \%$ (range, $22.18 \%-53.8 \%$ ). FES also seemed to have a positive orthotic effect on the PCI. Recently, another meta-analysis (6) was published where the therapeutic effect of FES on walking speed in stroke survivors was determined. A significant mean difference in walking speed of $.18 \mathrm{~m} / \mathrm{s}$ was found, indicating the effectiveness of FES treatment.

At present, only 1 randomized controlled trial (RCT) examined the effect of common peroneal nerve stimulation on walking speed in stroke survivors with chronic 
hemiplegia (7). The study reported a significant improvement of $20.5 \%$ in walking speed in the FES group, whereas the control group, who received physiotherapy (PT) only, showed a non significant improvement of 5.2\%. However, this RCT did not use an ankle-foot orthosis (AFO) as the control device. A comparison was made between walking with FES versus no treatment. In clinical practice, the conventional treatment of a drop foot is an AFO, which is most often a plastic support worn in the shoe to keep the ankle in a neutral position. An interesting aspect that has not been clarified yet is the additional value of the peroneal nerve stimulator in comparison with an AFO as the control device.

Surface-based FES is the common approach in the clinical setting, but there are several problems with this approach including difficulty with electrode positioning and skin allergy (3). Assuming that the drop foot requires a permanent solution, an implantable system might be considered. Potential advantages include stability of electrode position, easier donning and doffing of the system, and reduced pain and skin irritation. Several implantable systems have been developed in the past $(8,9)$. These were 1-channel stimulators, which did not allow for differential activation of peroneus and anterior tibial muscles for inversion-eversion balance post surgery. Accordingly, an implantable device with 2 independent channels was developed (10).

The aim of the present RCT was to determine the effect of an implantable two-channel peroneal nerve stimulator on walking speed and physical activity in comparison with the usual treatment in stroke survivors with a drop foot. We hypothesized that the intervention group would improve their walking speed by at least $.20 \mathrm{~m} / \mathrm{s}$, defined by Perry et al. (11) as clinically relevant over a device use period of over 6 months. Furthermore, we expected that the intervention group would show an increase in physical activity at the end of the trial. No changes in the control group were expected in both outcome measures. 


\section{Methods}

\section{Study Design}

We conducted this study as an RCT and the CONSORT statement was used to report the trial (12). All subjects were assessed 5 times in the gait laboratory. The baseline measurement took place about 1 week before the randomization procedure, and the follow-up measurements were performed $4,8,12$, and 26 weeks after the surgical procedure in the intervention group. Subjects assigned to the control group were measured in the same weeks as subjects assigned to the intervention group.

Walking speed, measured both by the six-minute walk test (6MWT) (13) and on a 10-m walkway, was defined as the primary outcome measure. The 6MWT was measured only during baseline, week 12, and week 26. Comfortable walking speed, measured on a 10m walkway, was measured during all assessments. Physical activity (14), the secondary outcome measure, was measured at baseline and at week 26 by monitoring a randomly selected group of subjects from both the intervention and control groups. Unfortunately, it was not possible to monitor all participating subjects, because only 2 activPAL systems ${ }^{1}$ were available during the trial.

All measurements were performed by the same examiners (AK, MT). Instructing the subjects in the intervention group on the proper use of the peroneal nerve stimulator and assessment of stimulation levels of the 2 output channels took place on the same day as the outcomes assessment. This was done for 2 reasons: (1) to keep the number of visits similar for both study groups so that the same amount of attention was paid to both groups; and (2) to save time and travel costs in the intervention group. If problems were experienced by the patients they were instructed to report them immediately, so that they could be resolved as soon as possible. Blinding of both the study personnel and participants was not possible due to the surgical procedures. All data were analyzed according to an intention to treat principle, which means that all participants in the trial were analyzed according to the treatment to which they were allocated, whether they received it or not.

\section{Participants}

Most subjects were recruited to the trial in response to an article in a local Dutch newspaper about the first results with the implantable stimulator in stroke survivors. Some patients were recruited through consultant and general practitioner referrals and in some cases on the advice of the physiotherapist treating the patient. Both 
recruitment strategies resulted in a selected sample of well-motivated stroke survivors. Because of the invasive nature of the treatment, selection of the most appropriate patients is very important. Therefore patients that were most likely to experience treatment success were selected. Table 3.1 shows the criteria for inclusion and exclusion in the trial.

Table 3.1 Selection criteria

\begin{tabular}{ll}
\hline Inclusion criteria & Exclusion criteria \\
\hline $\begin{array}{l}\text { Drop foot identified by an inability to achieve a } \\
\text { normal heel strike during walking }\end{array}$ & Age $<18$ year \\
$\begin{array}{l}\text { First hemiplegia of at least } 6 \text { months duration as a } \\
\text { result of a CVA with a stable neurology }\end{array}$ & $\begin{array}{l}\text { Passive dorsiflexion of the ankle }<5 \text { o with knee in } \\
\text { extension } \\
\text { Subject is an outdoor walker }\end{array}$ \\
$\begin{array}{l}\text { Medical conditions other than CVA, i.e. neurological, } \\
\text { rheumatic, cardio-vascular or systemic disorders } \\
\text { (including Diabetes Mellitus) limiting the function of } \\
\text { walking } \\
\text { Injury to deep and superficial peroneal nerve and } \\
\text { sciatic nerve } \\
\text { Any medical condition that would exclude the use of } \\
\text { able to give an informed consent } \\
\text { Not able to don and doff the equipment } \\
\text { Pregnancy }\end{array}$ \\
\hline
\end{tabular}

Subjects that fulfilled the selection criteria were admitted to the trial. One week after the baseline assessment, subjects were randomly allocated to either the intervention group or control group. Random allocation was performed in blocks of 2 subjects, to ensure a close balance of the numbers in each group. The randomization procedure was performed by an independent person. All patients were allowed to continue their usual PT sessions during the trial, which was recorded in a patient diary.

The experiment protocol was approved by the local medical ethics committee and the subjects gave their informed consent before participating in the experiments.

\section{The Stimulation System}

The implantable two-channel peroneal nerve stimulator ${ }^{2}$ consists of an external transmitter with a built-in antenna, a foot switch, and implantable components consisting of the stimulator, the 2 leads, and the bipolar intraneural electrodes (Figure 3.1) $(10,15)$.

Transmitter. The transmitter uses a single $40-\mathrm{mm}$ diameter transmission coil that transmits alternately on 2 frequencies. This switching results in a pulse repetition rate 
of $30 \mathrm{~Hz}$ on each channel. The amplitudes of the monophasic pulses modulated on each carrier wave are controlled separately. The transmitter weighs approximately $0.1 \mathrm{~kg}$ and is attached with a strap on the lateral side of the lower leg, over the site of the implant, just below the knee. A footswitch placed under the heel of the patient's foot inside the shoe determines the on-and-off switching of the stimulation. The transmitter battery is charged overnight.

Implanted stimulator. The implantable two-channel peroneal nerve stimulator is a passive device, receiving information carried by the radiofrequency signals and converting them into the stimulation pulses of the desired amplitude and frequency. The receiver block is approximately $33 \mathrm{~mm}$ in diameter and $6 \mathrm{~mm}$ thick. It contains 2 independent and galvanically separate electric circuits built on a ceramic substrate $29 \mathrm{~mm}$ in diameter. The 2 circuits are tuned to operate at different frequencies, namely, 1 and $2 \mathrm{MHz}$, allowing them to be individually controlled by the transmitter. This further reduces the risk of cross-talk between the channels. The electronic circuits of the receiver block are encapsulated in silicone rubber elastomer. The electrodes are placed under the epineurium of the nerve, providing good mechanical stability and, due to the proximity to the nerve fascicles, low stimulation currents can be used. The 2 cables that connect the receiver block to the electrode arrays are composed of 2 helically wound platinum wire conductors with enamel insulation. The intertwined helixes are encased in silicone rubber elastomer. The electrodes are surgically positioned at 2 distinct locations, which are determined during test stimulation with a hook electrode combined with visual inspection of the generated movement. One electrode is placed under the epineurium of the superficial peroneal nerve (SPN) (eversion) and the other under the epineurium of the deep peroneal nerve (DPN) (dorsiflexion). The stimulation pulses have an asymmetric biphasic charge balanced waveform.

Surgical procedure. For patients receiving the implant, we performed a presurgical nerve conduction velocity measurement to check the integrity of the deep and superficial branches of the peroneal nerve. The surgical procedure was performed under general or spinal anesthesia. The patient's knee was placed in a flexed position to relax the common peroneal nerve (CPN). An incision of approximately $50 \mathrm{~mm}$ along the course of the CPN was made, beginning just below the head of the fibulae. The CPN and its 2 branches, the DPN and the SPN, were visually identified. Nerve identity was checked using a surgical nerve stimulator and hooked electrodes. The response to 
stimulation of each nerve was checked at several stimulation sites, along and around the nerve. When the site for optimal responses was identified on both nerves during test stimulation, a small incision through the epineurium at each of the 2 nerves was made. At each of the 2 sites, an electrode was inserted underneath the epineurium, which was fixed in place using sutures or tissue col fixative, a surgical glue. Using tags, positioned approximately $15 \mathrm{~mm}$ from the end of the electrode, the leads were sutured to the fascia of the underlying tibialis muscle. The leads were arranged in such a way as to reduce as far as possible the possibility of mechanical loading on the electrode sites resulting from muscle activity. The receiver body was placed in a subcutaneous pocket. Several test stimulations took place before closing the wound. Two weeks after the surgery the wound was checked and a first test stimulation took place. In the third week, stimulation during walking was tested and the stimulator was taken home by the patient. The use of the stimulator was gradually increased over 2 weeks to prevent severe muscle pain and fatigue. After this period patients were allowed to use the system all day.

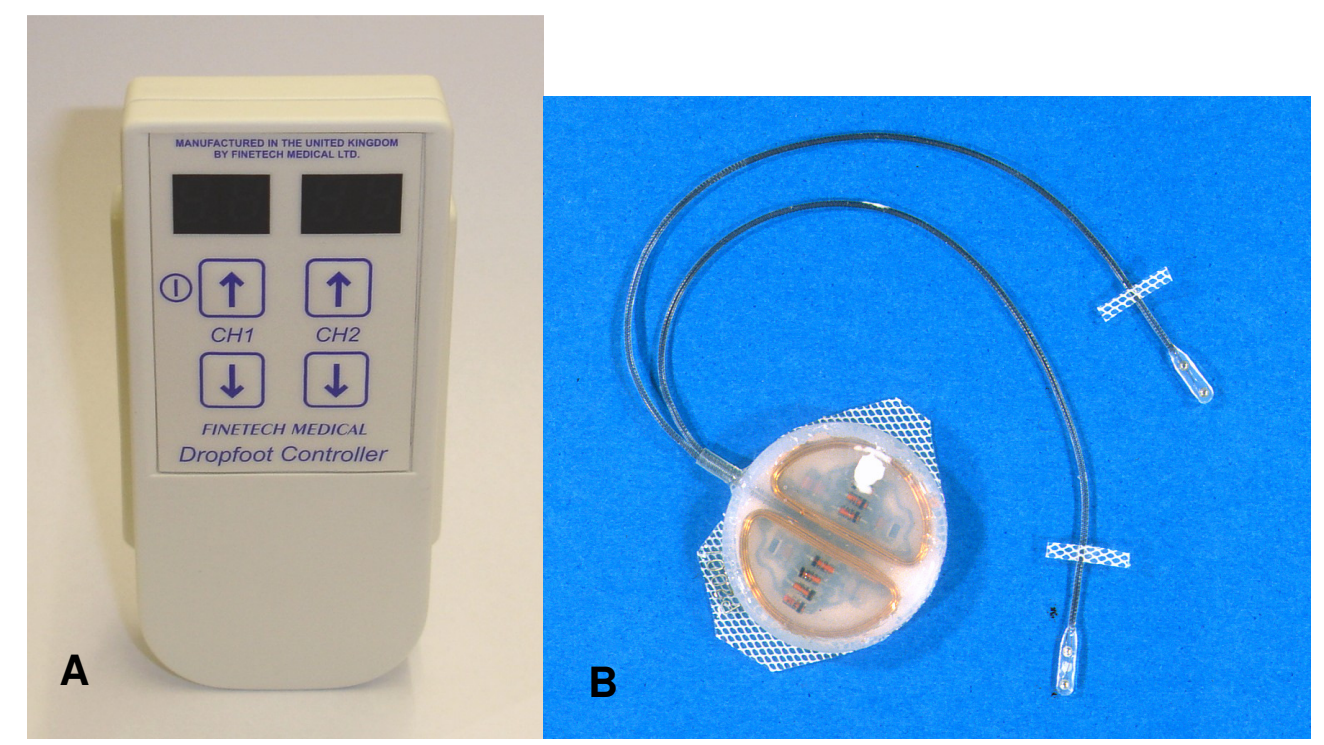

Figure 3.1 Implantable two-channel peroneal nerve stimulator: (A) external system and (B) implant 


\section{Measurements}

Assessment of 6MWT. The 6MWT was used to estimate the walking speed during daily activity. Butland et al. (16) reported that results of the 6MWT are highly reproducible and show moderate to strong correlations with comparable outcome measures. An oval course with known distance was clearly defined in the gait laboratory. The distance walked in 6 minutes at a comfortable walking speed was recorded using a stopwatch. Subjects were allowed to use a walking stick if necessary and this was recorded. The condition during baseline was the standard for the followup measurements. During baseline, all subjects walked with their conventional walking aid for the correction of their drop foot. During the follow-up measurements, the control group walked with their conventional walking aid and the intervention group walked with the implantable two-channel drop foot stimulator. Walking speed was calculated by dividing the walking distance by $360(6 \mathrm{~min}=360 \mathrm{~s})$.

Assessment of walking speed. We instructed patients to walk at a comfortable walking speed in the gait laboratory on a $10-\mathrm{m}$ walkway; no other instructions were given. Walking speed was measured automatically by using the Vicon system ${ }^{3}$, consisting of 2 infrared beams over a distance of $7.5 \mathrm{~m}$. To exclude the influence of acceleration and deceleration at the beginning and end of the walkway, $1.5 \mathrm{~m}$ were allowed at the start and finish of the walkway. Walking speed measured on a $10-\mathrm{m}$ walkway was found to be a valid, reliable and responsive outcome measure $(17,18)$. Control subjects were asked to perform the walk 4 times without and 4 times with their conventional walking aid during all measurements. At baseline, intervention group subjects were asked to walk 4 times without and 4 times with their conventional walking aid. During the follow-up measurements, they were asked to walk 4 times without and 4 times with stimulation. All subjects were allowed to use a walking stick if needed and this was recorded. The condition during baseline was the standard for the follow-up measurements. In each walking condition, the first walk was excluded from analysis. For each walking condition a mean walking speed was calculated by averaging the 3 remaining walking sessions.

Assessment of activity level. We used the activPAL professional to electronically monitor the level of activity in patients' home environment. The activPAL is an accelerometer based measurement device used to record subjects' primary physical activities (stepping, standing, sitting/lying) during their daily life. Data from the activPAL have been shown to be both valid and reliable (14). The device was fixed 
using an adhesive tape on the mid-line of the thigh, midway between hip and knee. Because only 2 activPAL systems were available during the trial, it was not possible to monitor all participating subjects. Therefore, 10 subjects of the control group and 11 subjects of the intervention group were randomly selected to be monitored over a 5-day period, with exception of the weekend, through all waking hours during 2 evaluation periods. The first evaluation period was performed at baseline and the second evaluation period was performed at week 26 . The selected outcome parameters were the percentage time spent on stepping, standing, and sitting/lying. Mean values were calculated by averaging the values found on the 5 recorded days.

\section{Statistical Analysis}

We performed a power analysis based on estimates that were obtained from the pooled analysis in a previous review from our group (5). The review intended to analyze the orthotic effect of FES on the improvement of walking in stroke patients with a drop foot. Data on walking speed of 4 clinical papers were pooled to estimate a mean difference \pm standard deviation (SD) of $.134 \pm .124 \mathrm{~m} / \mathrm{s}$. The following numbers were used for the power analysis: mean $1, .000 \pm .124$; mean $2, .134 \pm .124 ; \delta=.134 ; \alpha=.05$; and power, .80. The power calculation resulted in a number of 14 subjects in each group.

Baseline characteristics of the 2 groups were compared to evaluate the success of randomization. Walking speed parameters were tested for normality using the ShapiroWilks test, indicating a normal distribution for walking speed values $(P>05)$. We used linear mixed-model analyses to determine the overall orthotic effect of FES on both walking speed parameters when compared with the conventional treatment. An advantage of this method is that all available data could be included in the analysis, even if some data were missing. Group (FES, conventional treatment), time outcome assessments $(-6,4,8,12,26 \mathrm{wk})$, and the interaction between group and time were entered as terms in the model. The interaction is used to test differences between both groups in the change in outcome measured over time. Differences between and within both groups over the period between baseline and week 26 were evaluated. The model was also used by us to measure the strength of association between both walking parameters. Post hoc tests were performed for both walking speed parameters with Sidak-adjusted multiple comparisons. 
Because of the non-normal distribution of the activPAL data (Shapiro-Wilks test, $\mathrm{p}<.05)$, we used the Wilcoxon signed rank test to compare the activPAL data in both groups between both evaluation periods. The significance level $\alpha$ was set at .05 for all tests. All statistical analyses were performed with SPSS 4 for Windows. 


\section{Results}

\section{Participants}

Figure 3.2 shows the flow of participants throughout the study. Twenty-nine patients with drop foot due to cerebrovascular accident participated in the present study. Table 3.2 shows the characteristics of the participants. Subject characteristics were not very different between both groups, with the exception of mean time after stroke, which was longer for the intervention group. At baseline both groups also exhibited no significant difference in walking speed, assessed both by a $6 \mathrm{MWT}$ and by using a $10-\mathrm{m}$ walkway, indicating that the intervention and control group were matched.

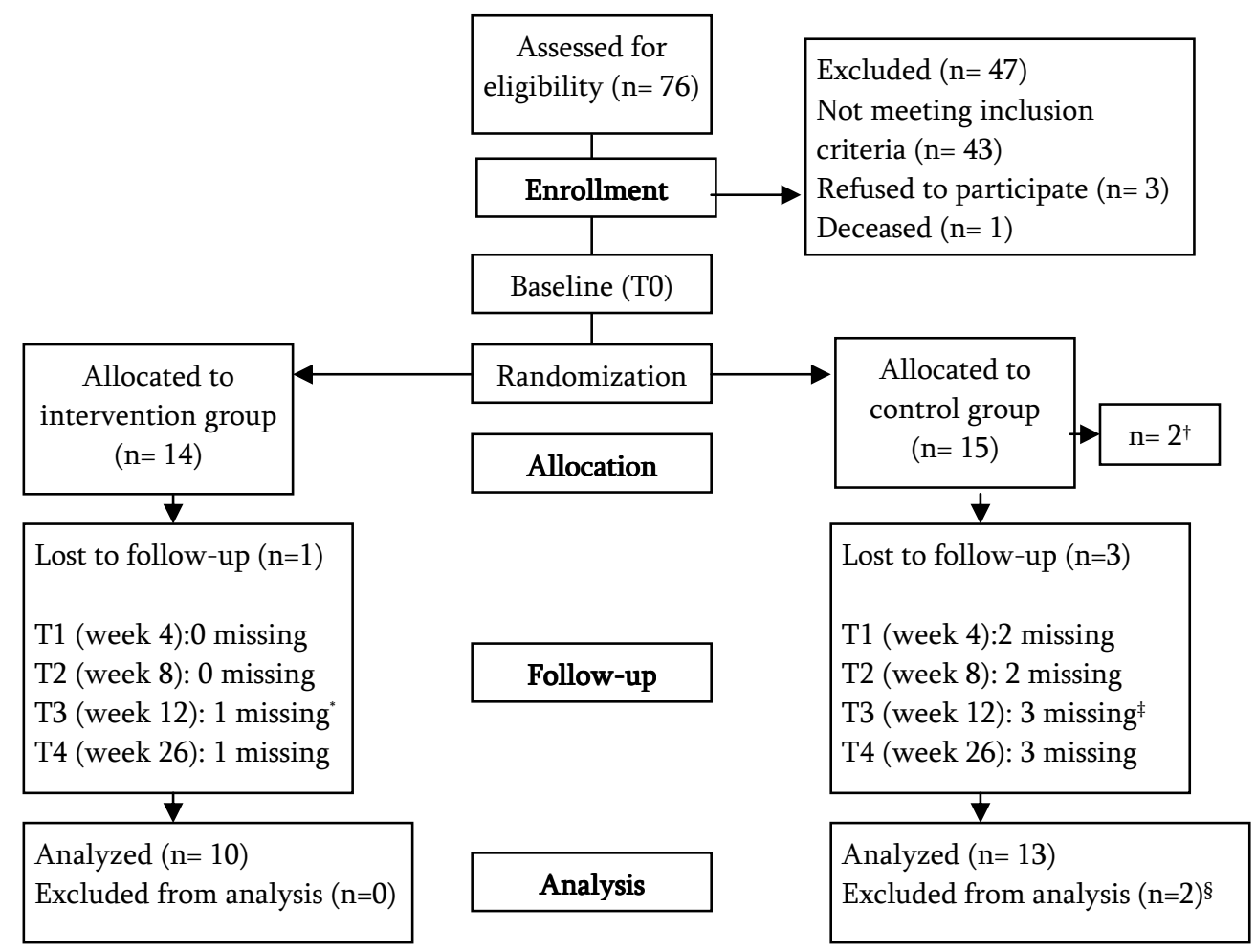

Figure 3.2 Flowchart of patients through the study

* Defective implant

${ }^{\dagger}$ Resultant of randomization procedure

₹ Psychologic issues not related to the study

$\S$ Excluded from analysis as a result of drop-out after randomization 
Table 3.2 Subject characteristics

\begin{tabular}{lcc}
\hline & Intervention group & Control group \\
\hline Number of subjects (drop outs) & $14(1)$ & $15(3)$ \\
Men & 10 & 10 \\
Women & 4 & 5 \\
Mean age (sd) & 55.2 years $(11.36)$ & 52.87 years $(9.87)$ \\
Mean time after stroke (sd) & 9.07 years $(9.29)$ & 5.67 years $(4.64)$ \\
Left side affected & 7 & 9 \\
Right side affected & 7 & 6 \\
\hline
\end{tabular}

In the intervention group 7 patients used a plastic AFO, 3 patients wore orthopedic shoes and 4 patients did not use a walking aid to correct their drop foot just prior to the start of the trial. In the control group all subjects wore a plastic AFO. Four subjects dropped out of the study, 1 woman in the intervention and 3 men in the control group. The implant of the subject in the intervention group who dropped out failed after functioning properly for about 10 weeks. An investigation of the explanted system showed that the failure was caused by a technical defect in the epineural electrode responsible for the dorsiflexion movement. Two subjects in the control group withdrew after the randomization procedure and the other subject dropped out in week 11, because of psychologic issues not related to the study. The remaining subjects in the intervention group did not report any technical failure of the stimulation system and continued to use the stimulator during the entire follow-up period.

At baseline, walking speed when using the conventional walking aid in the intervention group was determined by means of calculating the mean walking speed for the subjects who used a plastic $\mathrm{AFO}$ or orthopedic shoes ( $\mathrm{n}=10)$.

\section{Six-Minute Walk Test}

Figure 3.3 shows both the mean walking distance that was reached during the 6MWT in both groups and the calculated walking speeds. Because no difference in baseline values was present between both groups, no correction for baseline value was necessary in the analysis. 


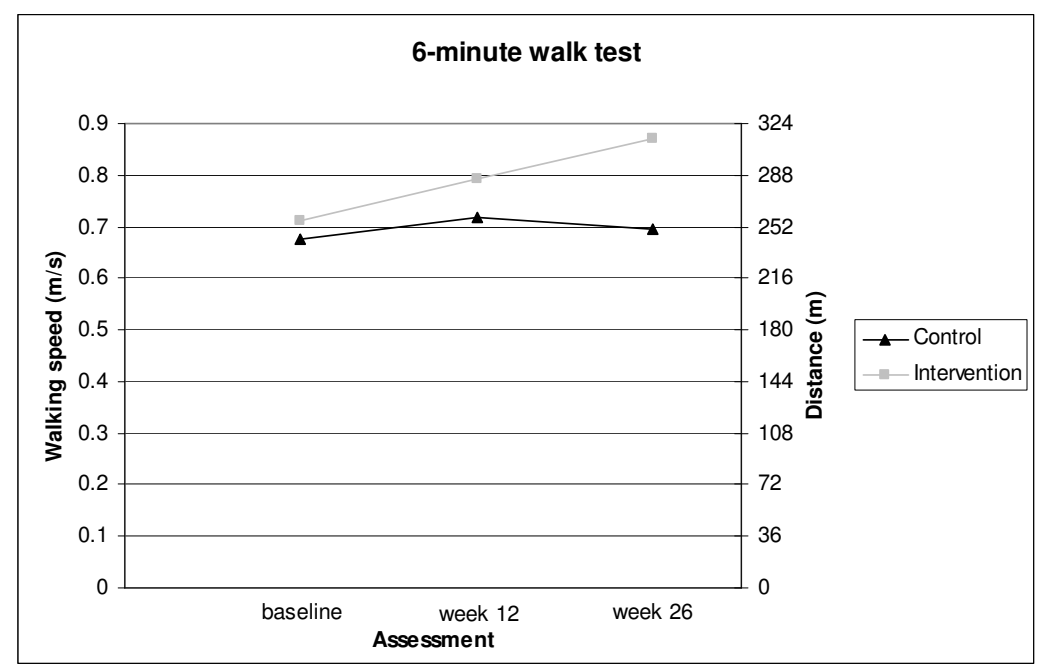

Figure 3.3 6MWT results measured in the intervention and control group: walking speed (left y-axis) calculated from distance (right y-axis)

A significant difference between both groups was found when all assessments were taken into the linear mixed model ( $\mathrm{p}=.010)$, showing a positive effect of FES on the performance of the 6MWT. At the first follow-up assessment (week 12), both the intervention and control group showed an improvement in the performance of the 6MWT. However, post hoc analysis showed that the change in walking speed at 12 weeks relative to baseline did not differ significantly between groups ( $\mathrm{p}=.49)$. At 26 weeks, the intervention group continued to show improvements, whereas the control group exhibited some deterioration. Post hoc analysis showed that the change in walking speed at 26 weeks relative to baseline now differed significantly between groups ( $\mathrm{p}=.049$ ).

\section{Walking Speed}

Figure 3.4 shows the results of the assessments for both groups obtained on all different walking speed conditions. Because there was no difference in baseline values, correction for baseline value was not included in the analysis.

When no walking device was used, no significant difference in walking speed between groups was found when all assessments were taken into the linear mixed model $(\mathrm{p}=.152)$. The changes within both the intervention and the control group over time, 
relative to their baseline values, were also not statistically significant $(\mathrm{p}=.812, \mathrm{p}=.112$, respectively).

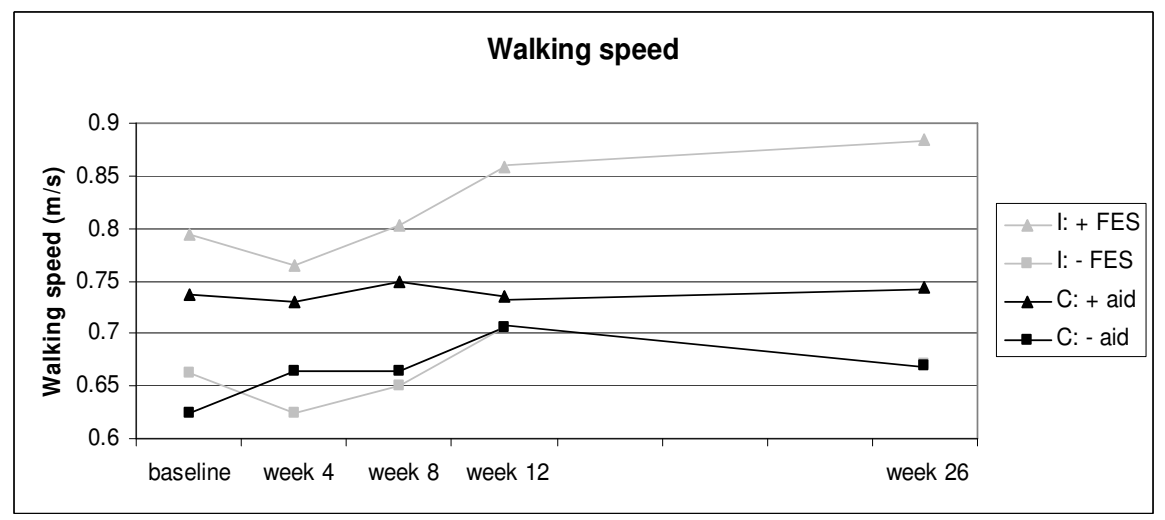

Figure 3.4 Walking speed (mean) measured on a 10-m walkway

C: control

I: intervention

When the control group used their walking aid and the intervention group used their FES, the linear mixed model indicated significant differences between groups ( $\mathrm{p}=.038$ ). Walking speed remained constant over time within the control group $(\mathrm{p}=.572)$. The intervention group showed a small deterioration in walking speed immediately after starting with the FES treatment (week 4), followed by an improvement in walking speed when FES was used for a longer period. Overall, when baseline was compared with the last follow-up assessment, the change in walking speed within the intervention group over time was statistically significant $(\mathrm{p}=.01)$.

When comparing only the last follow-up assessment with baseline between both groups, a trend toward statistically significant effect of FES on walking speed over time was found in comparison with the conventional treatment ( $\mathrm{p}=.097)$. At none of the follow-up assessments, where the use of FES in the intervention group was compared with the use of an AFO in the control group, did post hoc testing result in a statistically significant difference relative to baseline.

To examine the relation between both measurement techniques used to obtain comfortable walking speed, correlation coefficients were calculated. Both the 6MWT and the 10 -m walkway were performed during baseline, week 12 , and week 26 . The 
correlation coefficients were around .90 for all 3 assessments, indicating a strong relation.

\section{The activPAL Professional}

Table 3.3 shows the activPAL data measured in both groups. From the table it can be seen that the percentage time spent stepping deteriorated $3 \%$ and $0.8 \%$ in the intervention and control groups, respectively, when the first evaluation period was compared with the second. However, the difference between groups was not statistically significant $(\mathrm{p}=.13)$. The percentage time spent standing declined approximately $3 \%$ in the intervention group, whereas the control group improved $2 \%$. There was a trend toward statistically significant difference between groups $(\mathrm{p}=.06)$. Time spent on sitting/lying increased approximately $6 \%$ in the intervention group, whereas the control group declined approximately $1 \%$. The difference was statistically significant $(\mathrm{p}=.04)$.

Table 3.3 ActivPal data measured in intervention and control group (mean (sd))

\begin{tabular}{lccccccc}
\hline Group & Assessment & $\begin{array}{c}\text { Stepping } \\
\text { (\% time) }\end{array}$ & $\mathbf{p}$ & $\begin{array}{c}\text { Standing } \\
\text { (\% time) }\end{array}$ & $\mathbf{p}$ & $\begin{array}{c}\text { Sitting/ Lying } \\
\text { (\% time) }\end{array}$ & $\mathbf{p}$ \\
\hline Intervention & Baseline & 16.85 & .016 & 17.38 & .075 & 65.47 & .026 \\
& & $(9.24)$ & & $(7.34)$ & & $(15.29)$ & \\
& Week 26 & 13.89 & & 14.51 & & 71.44 & \\
& & $(6.63)$ & & $(7.51)$ & & $(11.22)$ & \\
Control & 10.41 & .213 & 14.36 & .386 & 75.25 & .953 \\
& Baseline & & & $(6.81)$ & & $(9.47)$ & \\
& \multirow{3}{*}{ Week 26 } & $9.68)$ & & 16.35 & & 74.08 & \\
& & $(5.06)$ & & $(7.51)$ & & $(10.5)$ & \\
\hline
\end{tabular}




\section{Discussion}

The primary aim of this study was to determine the effect of using an implantable twochannel peroneal nerve stimulator on comfortable walking speed in comparison with the usual treatment in stroke survivors with a drop foot. Walking speed was measured in 2 different ways: by measuring average speed during a $6 \mathrm{MWT}$ and by measuring speed on a $10-\mathrm{m}$ walkway.

The results of the present study show that FES results in a significant improvement in walking speed measured with the 6MWT when FES is used for a period of about 6 months as a treatment for drop foot in chronic stroke survivors. Furthermore, comfortable walking speed measured on a $10-\mathrm{m}$ walkway also increases significantly in the intervention group with regard to the control group during the trial when all follow-up assessments are taken into the analysis.

Walking speed was measured in 2 different ways. Some differences might have been expected. The patient may feel greater pressure to perform well during the $10-\mathrm{m}$ walkway, whereas the longer duration of the 6MWT might reflect the more natural cadence and velocity. Another possible source of difference might be the longer duration of the 6MWT, which might induce a fatigue effect. However, both measurements gave very similar results; no systematic differences were found.

In the systematic review of Kottink et al. (5) in which the effect of FES on walking speed in stroke survivors with a drop foot was evaluated, most of the included studies did not compare FES with the conventional orthotic treatment, but made a comparison between the conditions with and without FES. We intended to perform a more pragmatic trial. The present study is only the second RCT done on drop foot stimulation and the first RCT that examined the effect of an implantable drop foot stimulator.

All subjects included were stroke survivors that were in a chronic phase, so spontaneous recovery was not expected to be a confounder in the present study. This is confirmed by obvious lack of changes in the walking speed in the control group over time. The improvements measured in the intervention group can therefore be completely attributed to the FES treatment.

Another characteristic of our patient group was that they all had a relatively good walking function at the start of the trial, which is a result of our strict predefined inclusion and exclusion criteria. Richards et al. (19) described that to be independent in the community, a speed of $.80 \pm .18 \mathrm{~m} / \mathrm{s}$ is required. 
When looking at the walking speeds measured at baseline, one can conclude that all subjects in the trial satisfied this criterion. Most studies that examined the effect of peroneal nerve stimulation included subjects with a more impaired walking function. The control and intervention groups in the study by Burridge et al. (7) walked with a speed of .48 and $.64 \mathrm{~m} / \mathrm{s}$, respectively, at baseline. Patients included in the study by Bogataj et al. (20), consisting of acute, subacute, and chronic stroke survivors, showed a mean walking speed of .19 and $.23 \mathrm{~m} / \mathrm{s}$ for FES and control groups, respectively. In the literature, Wieler et al. (21) described that FES systems were of most benefit to subjects who walked very slowly. They explained that the smaller improvements among less impaired walkers were due to the fact they already had good control over many muscle groups. Consistent with this hypothesis, Ladouceur and Barbeau (22) reported a negative correlation between initial walking speed and the effect of FES. Thus, the inclusion of more severely impaired patients may be associated with a larger treatment effect. Although implantable stimulators have clear advantages with respect to the accuracy of stimulation and user comfort, it is also obvious that surgery is required and overall costs are considerably higher. Thus, candidates for the implantable system should first be given a trial period with a surface peroneal nerve stimulator to assess and appreciate the potential benefits of using electric stimulation. When these users then encounter specific problems that might be amenable to implantable systems such as poor electrode reliability, painful sensation, and difficulty with donning and doffing the system, implantation should be considered.

A slight deterioration in walking speed measured on a 10-m walkway was seen in the intervention group at the first follow-up. This can be explained by the anticipated inactive period and deconditioning after the implantation procedure. As the deterioration was observed with and without FES use, this explanation appears plausible. At the last follow-up assessment the intervention group continued to show improvements in walking speed, which suggests that a plateau has not been reached.

\section{Study Limitations}

A limitation of this study is the small sample size. This is reflected in the finding that FES resulted in a significant improvement of comfortable walking speed measured on a 10 - $\mathrm{m}$ walkway when all assessments were included in the linear mixed model ( $\mathrm{p}=.038$ ), but only a trend toward significance when baseline was compared with the last followup assessment $(\mathrm{p}=.097)$. The more data are included in the linear mixed model, the 
higher the power. Reaching sufficient power is often reported as a problem in studies performed in a rehabilitation setting.

\section{Clinical Relevance}

An interesting aspect to discuss is the clinical relevance of the results found in the present study. Clinicians can use this information to determine the effectiveness of the FES treatment. Perry et al. (11) reported that a difference of $.20 \mathrm{~m} / \mathrm{s}$ in walking speed with and without AFO was defined as clinically relevant. From figure 3.4 it can be seen that at the final follow-up assessment the control group shows a difference of $.07 \mathrm{~m} / \mathrm{s}$ in walking speed measured on a $10-\mathrm{m}$ walkway between the walking conditions with and without conventional walking aid. A difference of $.21 \mathrm{~m} / \mathrm{s}$ is found in the intervention group when walking with FES was compared with walking without FES, which is clinically relevant in accordance with Perry. Because the 6MWT was only performed while using a walking aid in both groups, it was not possible to test if these walking speed results were clinically relevant in accordance to Perry. However, another definition of clinical relevance was given by Burridge et al. (7), who considered a percentage change of $10 \%$ in walking speed to be functionally relevant. When looking at the walking speeds converted from the $6 \mathrm{MWT}$, the intervention and control groups show an improvement of $23 \%$ and $3 \%$, respectively. Thus, according to Burridge et al. (7), the results found in the intervention group are highly clinically relevant. When the comfortable walking speed results measured on a 10-m walkway are taken into consideration, an improvement of exactly $10 \%$ is found in the intervention group when using the FES system (baseline, $.80 \mathrm{~m} / \mathrm{s}$; week $26, .88 \mathrm{~m} / \mathrm{s}$ ). The control group did not show a change in walking speed during the trial when using their walking device. During both baseline and the last follow-up assessment walking speed was $.74 \mathrm{~m} / \mathrm{s}$. Some studies in the literature reported the same amount of improvement in walking speed measured on a 10-m walkway as was found in the present study. Waters et al. (8), who also studied the effect of an implantable stimulator in chronic stroke survivors, found a difference of $.24 \mathrm{~m} / \mathrm{s}$ in free cadence walking speed when walking with FES after surgery compared to walking without an orthosis before surgery. However, no control group was included in their study. The intervention group in the study performed by Bogataj et al. (20) showed an increase of $.22 \mathrm{~m} / \mathrm{s}$ in walking speed when FES was compared with walking without FES, whereas the control group improved $.03 \mathrm{~m} / \mathrm{s}$. Our 
hypothesis that the intervention group would show an improvement in walking speed that is clinically relevant is confirmed by the present study results.

The secondary aim of the present study was to measure physical activity by using the activPAL professional. Physical activity significantly decreases in the intervention group, as indicated by the decrease of time spent on stepping and more time spent on sitting/lying. This finding is remarkable as one would expect an increase of time spent on stepping, induced by the greater walking speed enabling people to walk greater distances.

Recently, Stein et al. (23) found that with surface stimulation the number of steps per day, as reflected in the number of delivered pulse trains, increased significantly over time. However, a direct comparison with the present study is hampered, because we investigated physical activity of subjects while using their conventional walking aid at baseline in comparison with physical activity while using the implantable device at week 26. Because all subjects in our study were in the chronic phase of stroke, they were all trained well in walking with their conventional walking aid. Physical activity at baseline is therefore expected to be at a higher level in the present study, making it more difficult to find differences in due time. Our results do suggest that patients do not change their averaged walking distance using FES whereas the increase in walking speed allows them to spend even less time in walking. Despite the decrease measured in daily activity, the overall results suggest that an implanted peroneal nerve stimulator is an effective treatment option for a select group of chronic stroke survivors with foot drop.

\section{Conclusion}

FES resulted in a significant and clinically relevant increase in walking speed. The intervention group showed an improvement of $23 \%$ in walking speed in comparison with a $3 \%$ improvement in the control group when walking speed was measured by means of the 6MWT. Comfortable walking speed, measured on a $10-\mathrm{m}$ walkway, was also significantly improved in favor of FES. In contrast to our expectations, the results found by the activPAL professional do suggest that the average walking distance did not change by applying the stimulator.

In conclusion the results suggest that the implantable two-channel peroneal nerve stimulator is a clinically relevant treatment option in a select group of chronic stroke 
survivors. Future studies might investigate the generalizability of the results to other stroke survivors and the relevance of the stimulation system in other patient categories with upper motor neuron drop foot problems. 


\section{References}

1. Burridge JH, Wood DE, Taylor PN, McLellan DL. Indices to describe different muscle activation patterns, identified during treadmill walking, in people with spastic drop-foot. Med Eng Phys 2001;23:427-34.

2. Taylor PN, Burridge JH, Dunkerley AL, et al. Clinical use of the Odstock dropped foot stimulator: its effect on the speed and effort of walking. Arch Phys Med Rehabil 1999;80:1577-83.

3. Taylor PN, Burridge JH, Dunkerley AL, et al. Patients' perceptions of the Odstock Dropped Foot Stimulator (ODFS). Clin Rehabil 1999;13:439-46.

4. Taylor P, Burridge J, Dunkerley A, et al. Clinical audit of 5 years provision of the Odstock dropped foot stimulator. Artif Organs 1999;23:440-2.

5. Kottink AI, Oostendorp LJ, Buurke JH, Nene AV, Hermens HJ, IJzerman MJ. The orthotic effect of functional electrical stimulation on the improvement of walking in stroke patients with a dropped foot: a systematic review. Artif Organs 2004;28:577-86.

6. Robbins SM, Houghton PE, Woodbury MG, Brown JL. The therapeutic effect of functional and transcutaneous electric stimulation on improving gait speed in stroke patients: a metaanalysis. Arch Phys Med Rehabil 2006;87:853-9.

7. Burridge JH, Taylor PN, Hagan SA, Wood DE, Swain ID. The effects of common peroneal stimulation on the effort and speed of walking: a randomized controlled trial with chronic hemiplegic patients. Clin Rehabil 1997;11:201-10.

8. Waters RL, McNeal D, Perry J. Experimental correction of footdrop by electrical stimulation of the peroneal nerve. J Bone Joint Surg Am 1975;57:1047-54.

9. Strojnik P, Acimovic R, Vavken E, Simic V, Stanic U. Treatment of drop foot using an implantable peroneal underknee stimulator. Scand J Rehabil Med 1987;19:37-43.

10. van der Aa HE, Bultstra G, Verloop AJ, et al. Application of a dual channel peroneal nerve stimulator in a patient with a "central" drop foot. Acta Neurochir Suppl 2002;79:105-7.

11. Perry J, Garrett M, Gronley JK, Mulroy SJ. Classification of walking handicap in the stroke population. Stroke 1995;26:982-9.

12. Altman DG, Schulz KF, Moher D, et al. The revised CONSORT statement for reporting randomized trials: explanation and elaboration. Ann Intern Med 2001;134:663-94.

13. Guyatt GH, Sullivan MJ, Thompson PJ, et al. The 6-minute walk: a new measure of exercise capacity in patients with chronic heart failure. Can Med Assoc J 1985;132:919-23.

14. Grant M, Ryan C, Tigbe W, Granat M. Validation of the activPAL ${ }^{\mathrm{TM}}$ activity monitor in measuring free-living physical activity. Biomechanics of the lower limb in health, disease and rehabilitation. Presented to: Salford's 3rd International Conference 2005 Sept 5-7; Salford, United Kingdom:196-7. 
15. Holsheimer J, Bultstra G, Verloop AJ, van der Aa HE, Hermens HJ. Implantable dual channel peroneal nerve stimulator. Presented to: Ljubljana FES Conference; 1993 Aug; Ljubljana, Slovenia:42-4.

16. Butland RJ, Pang J, Gross ER, Woodcock AA, Geddes DM. Two-, six-, and 12-minute walking tests in respiratory disease. Br Med J (Clin Res Ed) 1982;284:1607-8.

17. Richards CL, Olney SJ. Hemiparetic gait following stroke. Part II: Recovery and physical therapy. Gait Posture 1996;4:149-62.

18. Wade DT. Measurement in neurological rehabilitation. Oxford: Oxford Univ Pr; 1992.

19. Richards CL, Malouin F, Dean C. Gait in stroke: assessment and rehabilitation. Clin Geriatr Med 1999;15:833-55.

20. Bogataj U, Gros N, Kljajic M, Acimovic R, Malezic M. The rehabilitation of gait in patients with hemiplegia: a comparison between conventional therapy and multichannel functional electrical stimulation therapy. Phys Ther 1995;75:490-502.

21. Wieler M, Stein RB, Ladouceur M, et al. Multicenter evaluation of electrical stimulation systems for walking. Arch Phys Med Rehabil 1999;80:495-500.

22. Ladouceur M, Barbeau H. Functional electrical stimulation assisted walking for persons with incomplete spinal injuries: longitudinal changes in maximal overground walking speed. Scand J Rehabil Med 2000;32:28-36.

23. Stein RB, Chong S, Everaert DG, et al. A multicenter trial of a footdrop stimulator controlled by a tilt sensor. Neurorehabil Neural Repair 2006;20:371-9.

\section{Suppliers}

${ }^{1}$ PAL Technologies Ltd, 141 St James Rd, Glasgow, G4 OLT, UK.

2 STIMuSTEP; FineTech Medical Ltd, 13 Tewin Ct, Welwyn Garden City, Hertfordshire, AL7 1AU, England.

${ }^{3}$ Vicon, 14 Minns Business Park, West Way, Oxford, OX2 0JB, UK.

${ }^{4}$ Version 11.5; SPSS Inc, 233 S Wacker Dr, 11th Fl, Chicago, IL 60606 

Chapter 4

Therapeutic effect of an implantable peroneal nerve stimulator in subjects with chronic stroke and footdrop: a randomized controlled trial

Anke I.R. Kottink

Hermie J. Hermens

Anand V. Nene

Martin J.B. Tenniglo

Catharina G. Groothuis-Oudshoorn

Maarten J. IJzerman

Phys Ther. 2008;88:437-448.

Reprinted with permission 


\begin{abstract}
Footdrop, characterized by a person's inability to raise the foot at the ankle, is a common problem in patients with stroke. A randomized controlled trial was performed to determine the therapeutic effect, which is the effect that remains even after the stimulator has been removed, of using a new implantable, two-channel peroneal nerve stimulator for 6 months versus the conventional footdrop treatment.

Twenty-nine patients with chronic stroke and footdrop participated in the study. The mean time from stroke was 7.3 years $(\mathrm{SD}=7.3)$, and all subjects were community ambulators.

The functional electrical stimulation (FES) group received the implantable stimulation system for correction of their footdrop, while the control group continued using their conventional walking device (ie, AFO, orthopedic shoes, or no walking device). All subjects were measured at baseline and at weeks $4,8,12$, and 26 in the gait laboratory. The therapeutic effect of FES on the maximum value of the root mean square (RMSmax) of the tibialis anterior (TA) muscle with both flexed and extended knees and walking speed were selected as the primary outcome measures. The RMSmax of the peroneus longus (PL), gastrocnemius (GS), and soleus (SL) muscles with both flexed and extended knees and muscle activity of the TA muscle of the affected leg during the swing phase of gait were selected as secondary outcome measures.

A significantly higher RMSmax of the TA muscle with extended knee was found after using FES. No change in walking speed was found when the stimulator was not switched on. A significantly increased RMSmax of the GS muscle with both flexed and extended knees was found after using FES.

Functionally, no therapeutic effect of implantable peroneal nerve stimulation was found. However, the significantly increased voluntary muscle output of the TA and GS muscles after the use of FES suggests that there was a certain extent of plasticity in the subjects in this study.
\end{abstract}




\section{Introduction}

In most countries, patients with ankle dorsiflexor paresis, called "footdrop," are provided with an ankle-foot orthosis (AFO). An AFO provides mediolateral stability at the ankle during the stance phase of gait and improves the swing phase of the paretic leg by facilitating toe clearance and promoting heel-strike (1). Advantages of an AFO are that it is easy for the patient to apply, it provides consistent and stable support, and it is a relatively inexpensive device. A disadvantage is the limitation in mobility of the ankle joint during ambulation, which may inhibit recovery in the long term. In addition, patients often find AFOs to be uncomfortable or ineffective (2). Beckerman et al. (3) reported that half of their patients were not adherent in wearing an AFO and that two thirds of their patients were not satisfied with the use of an AFO, as measured with the Sickness Impact Profile. Literature about AFOs is not conclusive, and the effect of an AFO on daily activities remains unknown (4).

Functional electrical stimulation (FES) may be an alternative approach to correcting footdrop that works by stimulating the peroneal nerve during the swing phase of the gait cycle. Liberson and colleagues (5) were the first investigators who reported on the application of FES in patients with footdrop as a consequence of stroke. They noted that some patients with stroke continued to dorsiflex the foot while walking after stimulation was stopped. This effect also was reported by other investigators (6-8). These results suggest that, as a consequence of walking with FES, plastic changes occur in the nervous system. Different names have been used in the literature to describe this phenomenon (ie, "carryover effect," "therapeutic effect," and "motor relearning effect"). The definition given by Waters for this phenomenon is "a temporary increase in voluntary control observed immediately after electrical stimulation" (9).

Recently, a meta-analysis was published by Robbins et al. (10) on the therapeutic effect of FES with surface electrodes on gait speed in patients with stroke. Three controlled studies examining the effect of peroneal nerve stimulation (11-13) were included in the meta-analysis. By using a fixed effects model, a mean difference of $0.18 \mathrm{~m} / \mathrm{s}$ was found $(\mathrm{p}<.01)$, indicating that FES can produce significant sustained improvements in gait speed even after the stimulator is turned off. An earlier, more descriptive review by Burridge et al. (14) examined both the orthotic and therapeutic effects of surface electrodes using single or dual channels of stimulation as an intervention for footdrop. A carryover effect was found in some of the studies (6-8) included in their review. The underlying mechanism and the duration and extent of the effects were not specified, 
and the type of patient who might benefit from this intervention could not be identified. Their conclusion was that more work is needed to be able to answer these questions.

Surface-based FES is a common approach in the clinical setting, but there are several problems with this approach, especially difficulty positioning the electrode accurately with one hand and skin allergies (2). Assuming that footdrop requires a permanent solution, an implantable system might be considered. Potential advantages of an implantable system include stability of electrode position, easier donning and doffing of the system, and reduced pain and skin irritation.

To date, no randomized clinical trial has evaluated the therapeutic effect of an implantable peroneal nerve stimulator versus an AFO on functional recovery in patients with footdrop who are in the chronic phase of stroke. In a recent study by Kottink et al. (15), a significant orthotic effect on walking speed was found in subjects who received FES, both when measured with a 6-minute walk test and when measured on a $10-\mathrm{m}$ walkway.

The aim of the present study was to assess the therapeutic effect of an implantable, twochannel peroneal nerve stimulator in comparison with that of an AFO on motor and functional recovery, as measured by 3 different outcome measures. First, the maximum value of the root mean square (RMSmax) of the tibialis anterior (TA), peroneus longus (PL), medial gastrocnemius (GS), and soleus (SL) muscles of the affected leg were measured during the performance of maximal voluntary contractions (MVCs) with the knees in both flexed and extended positions. The RMSmax reflects the capacity of the muscle output in a static test condition. Second, the amount of activity of the TA muscle of the affected leg during the swing phase of gait (RMSswing) was measured. In order to study changes in a functional status, comfortable walking speed was selected as a third outcome measure. In addition, RMSmax of the TA muscle and measurements of walking speed were correlated to investigate a positive relationship between both outcomes.

Conceptually, the repetitive stimulation of the muscles during gait for long periods of time and, consequently, the large amount of feedback to the brain might promote functional recovery. Especially in the TA muscle, increased maximal muscle activity was expected after prolonged use of FES because much higher intensity levels of stimulation for this muscle were used by our subjects when compared to the PL muscle, the other muscle that was directly stimulated. In some cases, stimulation of the PL 
muscle was not necessary because no inversion of the foot was seen in these subjects during the swing phase of gait. Furthermore, we expected that the increased RMSmax of the TA muscle would result in increased walking speed when FES was switched off. 


\section{Method}

\section{Study Design}

The study was conducted as a randomized controlled trial, and the CONSORT statement was used to report the trial (16). All subjects were assessed 5 times in the gait laboratory of Roessingh Research and Development, Enschede, the Netherlands. The baseline measurement took place about 4 weeks before the randomization procedure, and the follow-up measurements were performed 4, 8, 12, and 26 weeks after the surgical procedure of the FES group (see "Subjects" section for explanation of group assignments). The subjects assigned to the control group were measured in the same weeks as subjects assigned to the FES group. With the exception of RMSswing of the TA muscle, which was measured only at baseline and at week 26, the other outcomes were measured during each follow-up assessment. All measurements were performed by the same examiners (AK, MT). Ambulation activity (in meters per day) of both groups at baseline was asked by a questionnaire that was designed by Taylor and colleagues (2).

The RMSmax of the TA, PL, GS, and SL muscles of the affected leg was measured during MVC while the subjects' knees were in both flexed and extended positions. While in both positions, the subjects were asked to perform an MVC in ankle dorsiflexion, plantar flexion, and eversion.

In the dynamic condition, both RMS swing of the TA muscle of the affected leg and walking speed were determined while subjects were asked to perform a $10-\mathrm{m}$ walk several times. Walking speed was measured in 2 different conditions. All subjects started walking 4 times without using their conventional walking device (ie, AFO, orthopedic shoes, or no walking device). Thereafter, the same test was performed by both the FES and control groups while walking with their conventional walking device. The RMSswing of the TA muscle was measured only in the first test condition, when no walking device was used.

Instructing the subjects in the FES group on the proper use of the peroneal nerve stimulator and assessment of stimulation levels of the 2 output channels took place on the same day as the outcomes assessment. This was done for 2 reasons: (1) to keep the number of visits similar for both study groups so that the same amount of attention was paid to both groups and (2) to save time and travel costs in the FES group. If the subjects experienced problems, they were instructed to report them immediately so that a solution could be found as soon as possible. Blinding of both the study personnel and 
participants was not possible because of the surgical procedures. All data were analyzed according to the intention-to-treat principle.

\section{Subjects}

Subjects were recruited in different ways. Most subjects were recruited for the study in response to an article in a local Dutch newspaper about the first results with the implantable stimulator in people who had survived a stroke. Some subjects were recruited through consultant and general practitioner referrals and in some cases on the advice of the physical therapist treating the subject. Table 4.1 shows the criteria for inclusion and exclusion in the study.

Table 4.1 Selection criteria

\begin{tabular}{ll}
\hline Inclusion criteria & Exclusion criteria \\
\hline $\begin{array}{l}\text { Drop foot identified by an inability to achieve a } \\
\text { normal heel strike during walking }\end{array}$ & Age $<18$ year \\
$\begin{array}{l}\text { First hemiplegia of at least } 6 \text { months duration as a } \\
\text { result of a CVA with a stable neurology }\end{array}$ & $\begin{array}{l}\text { Passive dorsiflexion of the ankle }<\text { 5o with knee in } \\
\text { extension } \\
\text { Subject is an outdoor walker }\end{array}$ \\
$\begin{array}{l}\text { Medical conditions other than CVA, i.e. neurological, } \\
\text { rheumatic, cardio-vascular or systemic disorders } \\
\text { (including Diabetes Mellitus) limiting the function of } \\
\text { walking } \\
\text { Injury to deep and superficial peroneal nerve and } \\
\text { sciatic nerve } \\
\text { Any medical condition that would exclude the use of } \\
\text { a surgical procedure or anesthetic } \\
\text { Not able to don and doff the equipment } \\
\text { Pregnancy }\end{array}$ \\
\hline
\end{tabular}

In total, 76 subjects were assessed for eligibility, and 47 subjects were excluded. Fortythree subjects did not fulfill the selection criteria, 3 subjects refused to participate, and 1 subject died during the period between the screening and the baseline measurement. Subjects who fulfilled the selection criteria were admitted to the study and 1 week after the baseline assessment were randomly allocated to either an FES group or a control group. Random allocation was performed in blocks of 2 subjects to ensure close balance of the numbers in each group. By means of a randomization list that was generated before the start of the study, it could be determined which subject of each block of 2 subjects was randomized to which treatment. The FES group received the implantable stimulator, and the control group continued using their conventional walking device for correction of their footdrop. The randomization procedure was performed by an 
independent individual. All subjects were allowed to continue their usual physical therapy sessions during the study, which they recorded in a diary. All subjects gave their informed consent before participating in the study.

Twenty-nine patients with footdrop due to a cerebrovascular accident (CVA) participated in the present study. Table 4.2 shows the characteristics of the participants. Subject characteristics were not very different between the 2 groups, with the exception of mean time after stroke, which was longer for the FES group. At baseline, both groups also exhibited no significant difference in walking speed, indicating that the FES and control groups were matched.

The FES group consisted of 14 subjects (10 male, 4 female). Seven subjects used a plastic AFO, 3 subjects wore orthopedic shoes, and 4 subjects did not use a walking aid to correct their footdrop at the start of the study. Two of the subjects who used a plastic AFO also used a cane during walking. At the baseline measurement, 4 subjects were receiving physical therapy ( 3 once a week and 1 twice a week). Seven subjects were affected on the left side, and 7 subjects were affected on the right side.

Table 4.2 Subject characteristics

\begin{tabular}{lccc}
\hline & FES group & Control group & P (Statistical test) \\
\hline Number of subjects (drop outs) & $14(1)$ & $15(3)$ & \\
Male & 10 & 10 & .78 (Chi-square test) \\
Female & 4 & 5 & \\
Mean age (sd) & 55.2 years & 52.87 years & .56 (T-test) \\
& $(11.36)$ & $(9.87)$ & .79 (Mann Whitney test) \\
Mean time from stroke (sd) & 9.07 years & 5.67 years & \\
& $(9.29)$ & $(4.64)$ & .59 (Chi-square test) \\
Physiotherapy & 4 & 3 & .98 (Chi-square test) \\
Walking distance per day & $10-100 \mathrm{~m}: 1$ & $10-100 \mathrm{m:}: 1$ & \\
& $100-500 \mathrm{~m}: 6$ & $100-500 \mathrm{~m}: 6$ & \\
& $500 \mathrm{~m}-1 \mathrm{km:}: 2$ & $500 \mathrm{~m}-1 \mathrm{km:}: 3$ & \\
Left side affected & $>1 \mathrm{~km}: 5$ & $>1 \mathrm{~km}: 5$ & .89 (Chi-square test) \\
Right side affected & 7 & 9 & \\
\hline
\end{tabular}

The control group consisted of 15 subjects ( 10 male, 5 female). All subjects wore a plastic $\mathrm{AFO}$, and 3 subjects also used a cane during walking. At the baseline measurement, 3 subjects were receiving physical therapy ( 2 once a week and 1 twice a week). Nine subjects were affected on the left side, and 6 subjects were affected on the right side. 
Four subjects ( 1 woman in the FES group and 3 men in the control group) dropped out of the study. The implant of the subject in the FES group who dropped out showed failure after having functioned properly for about 10 weeks. Investigation of the explanted system showed that the system failure was caused by a technical defect in the epineural electrode responsible for the dorsiflexion movement. The remaining subjects of the FES group did not experience technical failure of the stimulation system and continued to use the stimulator during the entire follow-up period. In the control group, 2 subjects withdrew after the randomization procedure, and the other subject dropped out in week 11 because of psychological problems (not due to the study). Thus, for the analysis we only had 2 dropouts, both in the control group. Despite contact with them, we could not obtain the last data.

At the baseline measurement, walking speed in the FES group while subjects used their conventional walking device was calculated as the mean walking speed for the subjects who used a plastic $\mathrm{AFO}$ or an orthopedic shoe $(\mathrm{n}=10)$.

\section{Stimulation System}

The implantable, two-channel peroneal nerve stimulator ${ }^{1}$ consists of an external transmitter with a built-in antenna, a footswitch, and an implantable part consisting of the stimulator, the 2 leads, and the bipolar intraneural electrodes $(17,18)$.

The transmitter uses a single 40-mmdiameter transmission coil that transmits alternately on 2 frequencies. This switching results in a pulse repetition rate of $30 \mathrm{~Hz}$ on each channel. The amplitudes of the monophasic pulses modulated on each carrier wave are controlled separately. The transmitter weighs approximately $0.1 \mathrm{~kg}$ and is attached with a strap on the lateral side of the lower leg, over the site of the implant, just below the knee. A footswitch placed under the heel of the subject's foot inside the shoe determines the on- and off switching of the stimulation. The battery of the transmitter is charged overnight.

The implantable, two-channel nerve stimulator is a passive device, receiving information carried by the radio frequency signals and converting them into the stimulation pulses of the desired amplitude and frequency. The receiver block is approximately $33 \mathrm{~mm}$ in diameter and $6 \mathrm{~mm}$ thick. It contains 2 independent and galvanically separate electrical circuits built upon a ceramic substrate $29 \mathrm{~mm}$ in diameter. The 2 circuits are tuned to operate at different frequencies (ie, 1 and $2 \mathrm{MHz}$ ), allowing them to be individually controlled by the transmitter. This further reduces the 
risk of "cross talk" between the channels. The electronic circuits of the receiver block are encapsulated in silicone rubber elastomer. One electrode is placed under the epineurium of the superficial peroneal nerve (eversion), and the other electrode is placed under the epineurium of the deep peroneal nerve (dorsiflexion). The stimulation pulses have an asymmetric, biphasic charge balanced waveform.

\section{Measurements}

MVC. Bipolar surface electromyographic (sEMG) activity of the TA, PL, GS, and SL muscles was recorded during maximal ankle dorsiflexion, plantar-flexion, and eversion contractions of the affected leg of the subjects. The electrodes (Arbo, type H93SG, silver-silver chloride gel electrodes, diameter $=16 \mathrm{~mm}$, interelectrode space $=23 \mathrm{~mm}^{2}$ ) were placed after shaving, scouring, and abrading the skin vigorously with an alcohol pad. All electrodes were placed according to international guidelines (19). A reference electrode was placed on the hand. The sEMG activity was recorded using an ambulant Porti5-16/ ASD system ${ }^{3}$ (input impedance $=10^{12} \Omega$, gain=20, 22-bit analog-to digital converter), which was bandpass finite impulse response filtered at 20 to $553 \mathrm{~Hz}$. The RMSmax was recorded in 2 positions: with flexed and extended knees. The primary reason for this was that the subjects had to activate their muscles in and out their synergistic pattern (20).

First, the subjects sat on a couch in an upright position while keeping their knees in 90 degrees of flexion. They were first asked to contract the ankle dorsiflexors maximally. The investigator held the subjects' heel firmly in one hand, while using the other hand to give resistance by straightening and extending the foot.

The same test was repeated for the plantar-flexion movement. During this test, the investigator held the subjects' heel firmly with one hand, while providing resistance with the other hand by flexing the foot. An eversion movement then was performed by the subjects. During this movement, the investigator cupped the subjects' heel in one hand and with the other hand gave resistance by grasping the distal portion of the forefoot and turning the sole of the foot toward the median plane. The subjects performed all movements 3 times for a period of 5 seconds, with a couple of seconds of rest between measurements.

Second, MVCs were performed while the subjects were lying supine on a couch. The subjects were asked again to perform maximal ankle dorsiflexion, plantar-flexion, and eversion contractions while their legs were kept in an extended position. They 
performed all movements 3 times for a period of 5 seconds, with a couple of seconds of rest between measurements. For each movement, a mean value was calculated by averaging the 3 values.

A customized software program was used to determine the RMSmax of the TA, PL, GS, and SL muscles. The RMSmax was calculated by taking the middle part of the electromyographic (EMG) signal to avoid start-up and slow-down effects of the MVC. Log transformation was applied to all EMG RMS data to produce a more normal distribution of values.

The same investigators measured sEMG activity during MVCs 5 times for all subjects. Because resistance was given by hand during the performance of the MVC experiment and no device was used to fix the ankle joint, intraclass correlation coefficients (ICCs) were calculated as a measure of the reliability of the MVC measurements.

TA muscle activity during swing phase of gait. Bipolar sEMG activity of the TA muscle, the primary ankle dorsiflexor muscle, of the affected leg was recorded during the performance of a 10-m walk. The TA muscle was selected because this muscle was directly stimulated by the implantable, two-channel peroneal nerve stimulator. Because the stimulator was active only during the swing phase of gait, as determined by a heel switch placed inside the shoe of the subjects, the RMS of the TA muscle was determined only during this phase of the gait cycle to determine a possible therapeutic effect of FES. To be able to define the gait cycle in the EMG recordings, footswitches were used to signal heel, midfoot, and toe contacts with the floor. They consisted of an aluminium conductive sheet covering the sole of the shoe in conjunction with a conductive rubber mat. Footswitch signals were taken from both feet together with the EMG signals and, by means of a synchronization signal given during each measurement, both signals could be linked together afterward. Subjects were asked to perform the walk 4 times without using their conventional walking device. They were instructed to walk at a comfortable speed; no other instructions were given. The use of a walking stick was allowed only when needed. To keep the test conditions the same during the trial, subjects who used a walking stick at the baseline assessment also had to use a walking cane at the follow-up assessment (week 26). A customized software program was used to determine RMSswing. For each walking session, the program automatically selected those parts of the gait cycle where the affected leg was in swing phase, from toe-off till 
heel-strike. The first walk was excluded from the analysis, and RMSswing was calculated by averaging the RMS values of the remaining 3 walking sessions.

Walking speed. Walking speed was measured automatically during the $10-\mathrm{m}$ walk with the VICON system ${ }^{4}$, consisting of 2 infrared beams over a distance of $7.5 \mathrm{~m}$. To exclude the influence of acceleration and deceleration at the beginning and end of the $10-\mathrm{m}$ walk, $1.5 \mathrm{~m}$ was allowed at the start and finish of the test. At each assessment, both the FES group and the control group were first asked to perform the walk 4 times without the use of their conventional walking device. Next, the same procedure was accomplished with use of their conventional walking device. The subjects were instructed to walk at a comfortable walking speed; no other instructions were given. To be able to measure a possible therapeutic effect of FES, the stimulation system was not used by the FES group during the experiment. During the trial, the FES group used the stimulator only in the home environment. There was a brief resting period between walking sessions. All subjects were allowed to use a walking cane, if needed, and this was recorded. The condition during the baseline assessment was the standard for the follow-up measurements.

In both walking conditions, the first walk was excluded from analysis. For each walking condition, a mean walking speed was calculated by averaging the speeds of the 3 remaining walking sessions. To investigate the absence or presence of a therapeutic effect in the FES group, walking speed measured with and without the conventional walking device at the baseline assessment was compared with the measurements of walking speed obtained during subsequent follow-up assessments. These results were compared with the results shown by the control group to examine whether a significant therapeutic effect of FES was present.

Correlation between RMSmax of the TA muscle and walking speed. A

positive relationship in the FES group was hypothesized between maximal muscle activity trained by FES and walking speed. If use of the peroneal nerve stimulator resulted in a therapeutic effect on both variables, high correlation coefficients must especially be seen in the TA muscle, since this is the muscle that was primarily stimulated. Therefore only correlations coefficients were calculated for this muscle. 


\section{Data Analysis}

A power analysis was performed based on estimates that were obtained from the pooled analysis in a previous review by our group (21). The review intended to analyze the orthotic effect of FES on the improvement of walking in patients with footdrop following a stroke. Data on walking speed in 4 clinical articles $(11,12,22,23)$ were pooled to estimate a mean difference of $0.134 \mathrm{~m} / \mathrm{s}(\mathrm{sd}=.124)$. The following numbers were used for the power analysis: in the control group, mean $=0$, $\mathrm{sd}=.124$; in the FES group, mean $=.134$, $\mathrm{sd}=.124$; delta $=.134$, alpha $=.05$, power $=.8$. The power calculation resulted in a number of 14 subjects in each group.

The Wilk-Shapiro test was used to test all outcome measures for normality. The RMS values were $\log$ transformed if a non-normal distribution was found. If, after log transformation, a non-normal distribution still was present, a nonparametric test was used.

MVC. A linear mixed model was used to test for differences in MVC between groups. Because a non-normal distribution was found only for the TA muscle $(\mathrm{p}<.05)$, a nonequivalent of the linear mixed model (the nonparametric factorial model for repeated measurements described by Brunner et al. (24)) was used to analyze the RMSmax of the TA muscle. Group (FES and control), time outcome assessments (baseline and 4, 8, 12, and 26 weeks), and the interaction between group and time were entered as terms in both the parametric and nonparametric models. The interaction was used to test for differences between groups in the change in RMSmax measured over time. Differences between and within groups over the period between the baseline assessment and the follow-up assessment at week 26 were evaluated. For each muscle, the ICC of the MVC measurements within subjects was calculated using a linear mixed model, with a random intercept per subject and with time, treatment, and their interaction as fixed factors (25).

TA muscle activity during swing phase of gait. The RMSswing was normally distributed ( $p>05$ ); therefore, a paired-samples $t$ test was used to compare baseline values with the values found at the week- 26 follow-up assessment.

Walking speed. Differences in walking speed between groups were analyzed with a linear mixed model because walking speed was normally distributed ( $p>05)$. The same 
terms were entered in this model in comparison with the model used for MVC. The strength of a positive relationship between the RMSmax of the TA muscle trained by FES and walking speed was tested by a Pearson correlation coefficient for each subject. The significance level was set at $\alpha=.05$ for all tests. The linear mixed model analyses, the paired-samples $t$ test, and the Pearson correlation coefficient were performed with SPSS version 11.5 for Windows 5 . The nonparametric factorial model for RMSmax of the TA muscle was performed with the statistical program $\mathrm{R}(26)$. 


\section{Results}

MVC

Test-retest reliability. Intraclass correlation coefficients were calculated as a measure of the reliability of the MVC measurements. The ICCs for the RMSmax of the TA, PL, GS, and SL muscles over time were .77, .66, .72, and .57, respectively. The mean RMSmax of the TA, PL, GS, and SL muscles in the FES and control groups with flexed and extended knee are summarized in Figure 4.1.
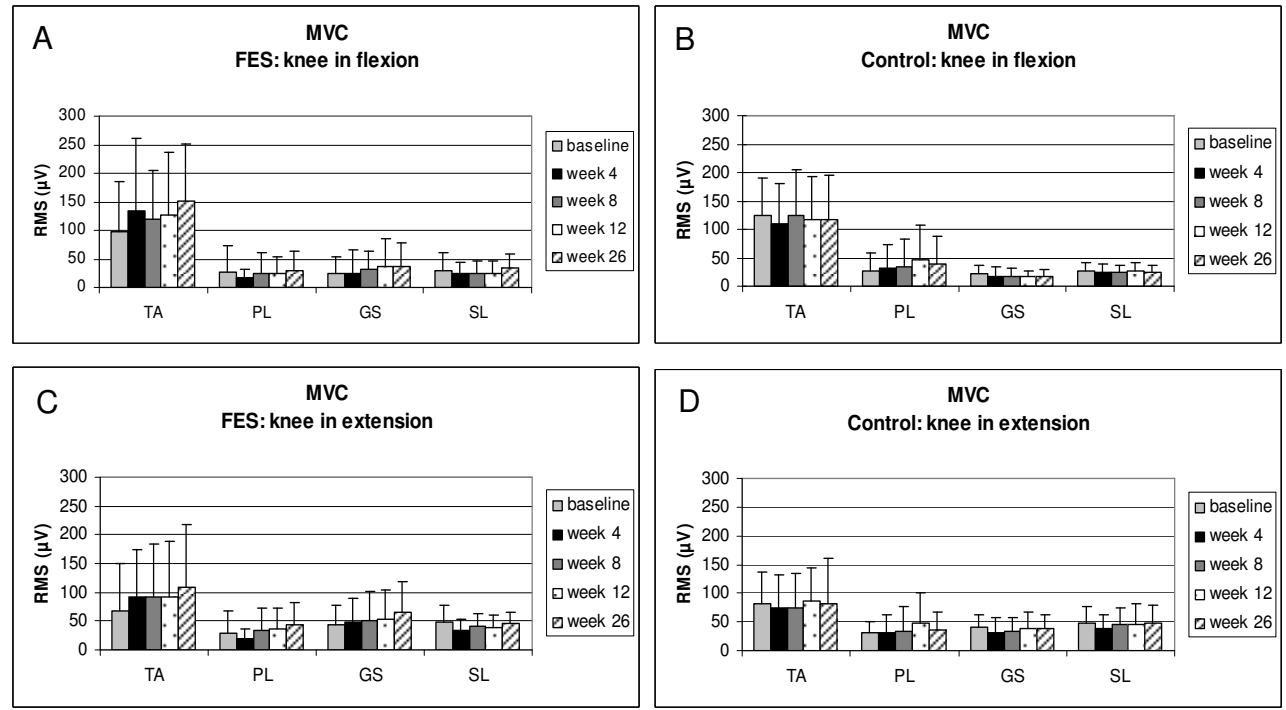

Figure 4.1 Mean maximal value of the root mean square (RMSmax) (in microvolts) of the tibialis anterior (TA), peroneus longus (PL), medial gastrocnemius (GS), and soleus (SL) muscle in the functional electrical stimulation (FES) group and the control group with the knee in flexion (A: FES group, B: control group) and with the knee in extension (C: FES group, D: control group), as measured by maximal voluntary contraction

Knee in flexion. As shown in Figures 4.1A and 4.1B, the RMSmax values of all muscles were very similar in both groups at the baseline assessment, with exception of the TA muscle, in which a higher RMSmax was found as in the control group. During the trial, the FES group showed higher RMSmax values for the TA muscle, increasing from $97 \mu \mathrm{V}$ at the baseline assessment to $150 \mu \mathrm{V}$ at the last follow-up assessment (Figure 4.1A). The RMSmax values in the control group stayed at the same level during the trial, around $120 \mu \mathrm{V}$ (Figure 4.1B). An almost significant value was found when calculating the change in RMSmax of the TA muscle over time between groups ( $\mathrm{p}=.058)$. The RMSmax 
for the PL muscle stayed rather constant over time in the FES group, and the control group seemed to improve slightly during the trial, although no significantly different change in RMSmax of the PL muscle over time between groups was found ( $\mathrm{p}=.860)$. The FES group showed an improvement in RMSmax of the GS muscle, from 25 to $38 \mu \mathrm{V}$. A small deterioration from 22 to $17 \mu \mathrm{V}$ in RMSmax was shown by the control group during the subsequent assessments. This resulted in a significant difference in RMSmax of the GS muscle over time between the FES and control groups ( $\mathrm{p}=.002)$. Both the FES and control groups showed a rather constant RMSmax for the SL muscle, not resulting in a significantly different change between groups over time $(\mathrm{p}=.330)$.

Knee in extension. Behavior for the RMSmax of the TA muscle in the extended knee position was similar to that during the flexed knee position (Figures $4.1 \mathrm{C}$ and 4.1D). The FES group showed an increase in RMSmax from 67 to $109 \mu \mathrm{V}$, and the RMSmax of the control group did not change during the study, staying around $81 \mu \mathrm{V}$. A large significant difference in the RMSmax of the TA muscle over time between groups was found ( $\mathrm{p}=.006$ ). The RMSmax of the PL muscle in the FES group increased slightly, from 29 to $45 \mu \mathrm{V}$. A small increase, from 32 to $36 \mu \mathrm{V}$, was found in the control group. No significantly different change over time was found in the RMSmax of the PL muscle between groups ( $\mathrm{p}=.208$ ). In addition, for the GS muscle, an increase in RMSmax, from 43 to $66 \mu \mathrm{V}$, was shown in the FES group, whereas the RMSmax of the control group did not change and stayed around $39 \mu \mathrm{V}$. The change in RMSmax over time between groups, therefore, was significantly different $(\mathrm{p}=.035)$. Both groups showed an RMSmax of $48 \mu \mathrm{V}$ for the SL muscle at the baseline assessment, which stayed constant during the study in both groups. Thus, no significantly different change in RMSmax of the SL muscle was found over time between the FES and control groups ( $\mathrm{p}=.654$ ).

\section{TA Muscle Activity During Swing Phase of Gait}

Figure 4.2 shows an example of the RMSswing during a walking session of a subject with left hemiplegia. At the baseline assessment, the FES and control groups showed mean RMSswing values of $54 \mu \mathrm{V}$ and $66 \mu \mathrm{V}$, respectively. During the follow-up assessment, the RMSswing in the FES group remained rather constant, showing an RMS of $58 \mu \mathrm{V}$ ( $\mathrm{p}=.706$ ). In the control group, an RMSswing value of $45 \mu \mathrm{V}$ was found, resulting in a significant decline $(\mathrm{p}=.036)$. 


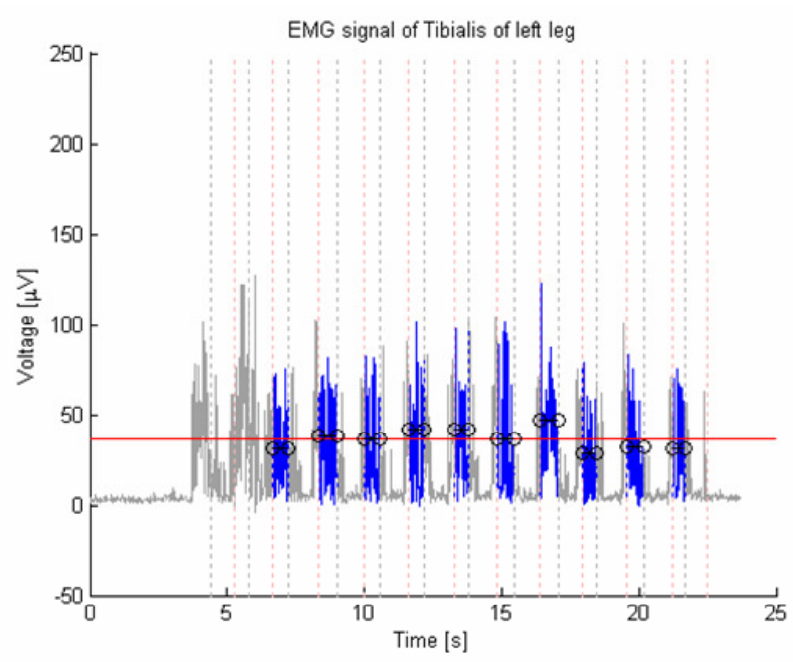

Figure 4.2 An example of the determination of amount of activity of the tibialis anterior (TA) muscle of the affected leg during the swing phase of gait (RMSswing) in a subjects with left hemiplegia The first dashed vertical line of the electromyographic signal represents toe-off and the second dashed vertical line represents heel-strike of the left affected leg. For every swing phase, a mean root mean square (RMS)

value was calculated by the program. The horizontal line represents the mean RMS value of the TA muscle of all swing phases during a walking session.

\section{Walking Speed}

Figure 4.3 shows the walking speed results of the subsequent assessments for both the FES and control groups when walking with and without a conventional walking aid. No correction for baseline was necessary in the analysis because no differences in baseline values were present between groups. Walking speed remained rather constant in both groups when no walking aid was used. The change in walking speed without a walking aid within the FES group over time, therefore, was not significantly different from that of the control group $(\mathrm{p}=.152)$. When the conventional walking aid was used, the walking speed results at the end of the study were comparable to the results at the baseline assessment in both groups. No significant difference over time was found when the 2 groups were compared with each other $(\mathrm{p}=.238)$. 


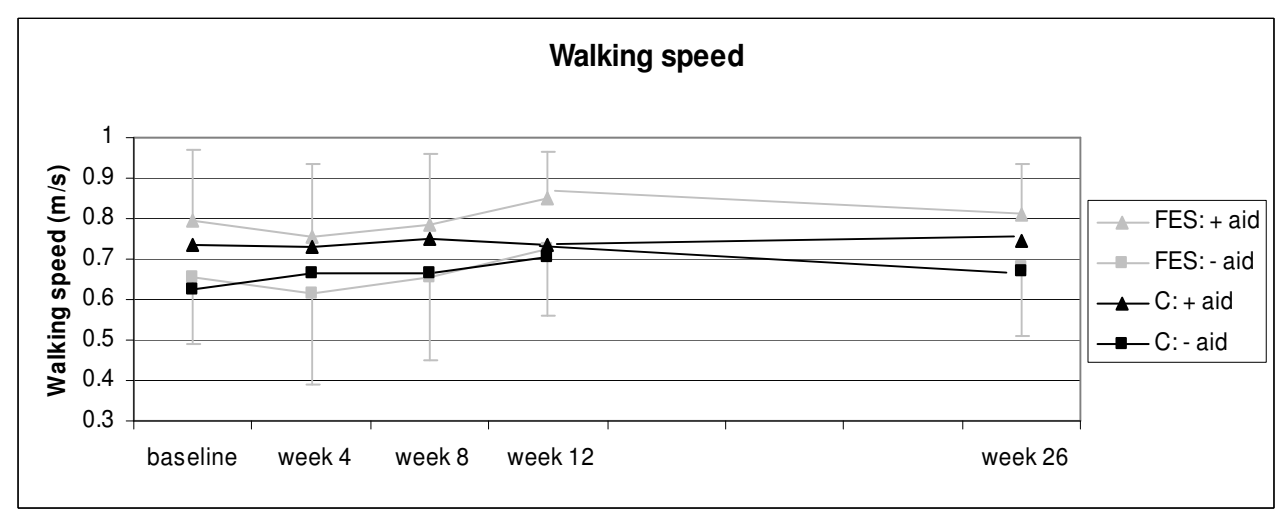

Figure 4.3 Mean walking speed measured in the functional electrical stimulation (FES) and control (C) group both during walking with and without their conventional walking aid (ankle-foot orthosis, orthopedic shoes, or no assistive device)

Error bars (SD) are shown only for the FES group because the error bars for the control group were very similar.

\section{Correlation Between RMSmax of the TA Muscle and Walking Speed}

Table 4.3 shows the Pearson correlation coefficients (range and median) found for the TA muscle in both the FES and control groups. Both variables were measured in 2 conditions: RMSmax with flexed and extended knee and walking speed with and without the use of an AFO. The subjects in both groups showed large differences in correlation coefficients calculated for all conditions, as reflected by the broad ranges found for both groups. Low median correlation coefficients were shown by both groups between RMSmax of the TA muscle and walking speed.

Table 4.3 Pearson correlation coefficients between maximum value of the root mean square (RMSmax) of the tibialis anterior muscle, measured with both flexed and extended knees, and walking speed, measured without and with an ankle-foot orthosis (AFO) in the functional electrical stimulation (FES) and control group: range (median)

\begin{tabular}{lcc}
\hline RMSmax & \multicolumn{2}{c}{ Walking speed } \\
\hline Knee in flexion & Without AFO & With AFO \\
FES & $-0.90-0.97(0.33)$ & $-0.90-0.84(-0.07)$ \\
Control & $-0.40-0.83(0.14)$ & $-0.87-0.53(0.14)$ \\
Knee in extension & Without AFO & With AFO \\
FES & $-0.83-0.60(-0.20)$ & $-0.80-0.76(-0.21)$ \\
Control & $-0.99-0.93(0.14)$ & $-0.73-0.73(0.07)$ \\
\hline
\end{tabular}




\section{Discussion}

The results of the present study show that use of an implantable FES device over a 6month period did not result in a change in self-selected walking speed when the device was turned off in subjects with chronic stroke and footdrop. In addition, no change in TA muscle activity was observed during the swing phase of walking, indicating no taskspecific learning for TA muscle activation. However, during the static test condition, consisting of voluntary activation

of the muscles, some interesting results were found, suggesting a certain extent of plasticity in motor control.

Streiner and Norman (27) recommended a reliability value of .70 when a scale is used for research purposes. For both the TA and GS muscles, acceptable test-retest reliability was found for the MVC tests (.77 and .72, respectively). An ICC of .66 was found for the PL muscle, and a relatively low ICC of .57 was found for the SL muscle. The voluntary muscle output of the TA and GS muscles was increased after using the stimulation system for a period of 6 months, suggesting a training effect of FES.

The RMSmax of the TA muscle was clearly higher with flexed knee in comparison with extended knee in both groups. This typical pattern can be explained by the flexion synergy that often is seen in patients with stroke. When the hip and knee are flexed, it is easier for patients with stroke to also bring their ankle into a flexed position, resulting is a dorsiflexion movement (20). Similarly, plantar flexion of the ankle must be easier to perform by patients while their hip and knee are in an extended position. When examining the GS and SL results, both groups showed a higher RMSmax with extended knee. Functional electrical stimulation seemed to have the largest positive effect on maximal muscle activity produced by the TA and GS muscles at both positions.

An explanation for the increased TA muscle activity was given by Miller and Light (28), who described that increased muscle activity can be accomplished in several ways, such as increasing the number of activated motor units, increasing the rate of activation, or increasing the synchronization of activation. In addition, as a result of FES training, the muscle fiber diameter may have been increased. To explain the therapeutic effect of FES on the TA muscle, Rushton (29) stated that the antidromic impulse in motor nerve fibers will reach the anterior horn cell. If the corticospinal-anterior horn cell synapse is a Hebb-type modifiable synapse, which means that it is strengthened by the coincidence of presynaptic and postsynaptic activity, then FES, combined with coincident voluntary effort through a damaged pyramidal motor system, could help to 
promote restorative synaptic modifications at the anterior horn cell level. Thus, electrical stimulation applied at rest is not expected to have any effect. Therefore, footdrop stimulation in particular, where stimulation is always applied at the time the automatic toe lift command is generated in the brain, might be successful in promoting functional recovery in patients. In contrast, plastic AFOs and orthopedic shoesstandard devices used for the correction of footdrop-limit the mobility of the ankle joint during ambulation and may further negatively affect recovery.

From the MVC results of the present study, it is not possible to conclude which mechanism was responsible for the increased maximal TA muscle activity. Experiments with multi-channel array electrodes may be performed in the future to assess motor control by measuring the number of motor unit action potentials (MUAPs) per second, which reflects the product of the number of motor units and their firing rate, and to examine the pattern and distribution of MUAPs following MVC more comprehensively $(30,31)$. With this measurement technique, it is possible to investigate whether the increased muscle activity was caused by a local training effect of the stimulated muscle or by a change in motor control (eg, an increased number of motor units).

Yan et al. (32) examined the direct effect of stimulation by measuring MVC of the ankle dorsiflexors and plantar flexors to determine whether FES in combination with standard care, consisting of physical therapy and occupational therapy, was more effective in promoting motor recovery of the lower extremity in subjects with acute stroke than placebo stimulation with standard care or standard care alone. A significantly increased MVC torque of the affected TA muscle was found, together with an improved plantarflexion torque at week 3 , after stimulation of the quadriceps femoris, hamstring, TA, and GS muscles 30 minutes a day, 5 days per week, for 3 weeks. The finding that the GS muscle, which is the antagonist of the TA muscle, also showed a significantly higher RMSmax with flexed and extended knees was not expected, because this muscle was not directly stimulated. However, these results also were found by Stefanovska et al. (33) They measured maximal isometric torque during voluntary contraction in dorsiflexion and plantar flexion in patients with hemiplegia with an implanted peroneal stimulator. After 6 months of stimulation, voluntary control was improved, particularly in the stimulated TA muscle but also in the antagonistic triceps surae muscle. This increased voluntary control can be attributed to decreased tonic activity in both the TA and triceps surae muscles, another parameter measured in their study. 
In contrast to our expectations, the results showed that FES used for a period of about 6 months had no therapeutic effect on walking speed. Walking speed is an outcome measure that often is used to measure whether a therapeutic effect is present after using electrical stimulation. Recently, a mean increase of $0.18 \mathrm{~m} / \mathrm{s} \quad(\mathrm{p}<.01)$ was found by Robbins et al. (10) in their systematic review. They reported that large effect sizes were found in FES studies examining subjects in the subacute stage of recovery, whereas relatively small effect sizes were found in FES studies examining subjects in the chronic stage of recovery. Robbins et al. examined 4 FES studies $(12,13,22,34)$ that included patients in the chronic phase of stroke whose mean time from stroke was about 37 months. In the present study, the mean times from stroke in the FES and control groups were 109 and 68 months, respectively. The chronic status of our subjects, therefore, may be a possible explanation for our finding of no significant therapeutic effect of FES on walking speed.

A positive relationship was hypothesized between maximal TA muscle activity and walking speed in the FES group. The low median correlation coefficients found in both groups however, suggest that no relationship exists between these 2 parameters. An explanation can be found when one considers that the prime function of the TA muscle consists of lifting the foot during the swing phase of gait, which will require an amount of activity and is unlikely to be linearly related to the walking speed. More likely is that, below a certain required activity level, TA muscle activity will affect the quality of the movement and, therefore, will show some relationship with walking speed. However, when a person is able to generate this amount of activity, an increase in the activity will not affect the walking speed because it is not directly related to the propulsion of the body. For the calf muscle, for example, a positive linear relationship with walking speed seems more plausible because of its propulsive function. Although the increased ability to voluntarily activate the TA muscle did not result in an improvement in walking speed, the higher lifting of the foot may give patients more confidence in their ability to walk without stumbling.

\section{Study Limitations}

This study had a number of limitations. The sample size was relatively small, which further limits generalizability to the broader population with stroke and placed the study at risk for false-negative results (type II error). Blinding of both the study personnel and patients was not possible because of the invasive FES treatment, which 
may have biased the study in favor of the FES group. Regarding the difference in AFO use between the FES and control groups, it was a coincidence that, after randomization, all control subjects used an AFO, whereas this was not the case in the FES group. There is no evidence, from the limited measurements that we made, that there were any significant differences across groups at the baseline assessment. Most subjects in the FES group had been prescribed an AFO in the past, but they rejected its use in daily practice. Differences in TA muscle activity also were observed between the groups in the baseline condition, although the differences were not statistically significant. The FES group showed lower baseline RMS values of the TA muscle while their knee was both in flexion and in extension compared with the control group.

\section{Conclusion}

Functionally, no therapeutic effect of implantable peroneal nerve stimulation was found, reflected by no change in walking speed when no stimulator was used. However, the significantly increased voluntary muscle output of the TA and GS muscles during a static task after the use of FES suggests that there is a certain extent of plasticity in people with chronic stroke and footdrop who are community ambulators. 


\section{References}

1. Leung JMA. Impact of ankle-foot orthosis on gait and leg muscle activity in adults with hemiplegia: systematic literature review. Physiotherapy 2003;89:39-55.

2. Taylor PN, Burridge JH, Dunkerley AL, et al. Patients' perceptions of the Odstock Dropped Foot Stimulator (ODFS). Clin Rehabil. 1999;13:439-446.

3. Beckerman H, Becher J, Lankhorst GJ, Verbeek AL. Walking ability of stroke patients: efficacy of tibial nerve blocking and a polypropylene ankle-foot orthosis. Arch Phys Med Rehabil. 1996;77:1144-1151.

4. de Wit DC, Buurke JH, Nijlant JM, et al. The effect of an ankle-foot orthosis on walking ability in chronic stroke patients: a randomized controlled trial. Clin Rehabil. 2004; 18:550-557.

5. Liberson WT, Holmquest HJ, Scot D, Dow M. Functional electrotherapy: stimulation of the peroneal nerve synchronized with the swing phase of the gait of hemiplegic patients. Arch Phys Med Rehabil. 1961; 42:101-105.

6. Carnstam B, Larsson LE, Prevec TS. Improvement of gait following functional electrical stimulation, I: investigations on changes in voluntary strength and proprioceptive reflexes. Scand J Rehabil Med. 1977;9:7-13.

7. Stefancic M, Rebersek M, Merletti R. The therapeutic effect of the Ljubljana functional electronic brace. Eur Medicophys. 1976;12:1-9.

8. Merletti R, Zelaschi F, Latella D, et al. A control study of muscle force recovery in hemiparetic patients during treatment with functional electrical stimulation. Scand J Rehabil Med. 1978;10:147-154.

9. Waters RL. The enigma of “carry-over.” Int Rehabil Med. 1984;6:9-12.

10. Robbins SM, Houghton PE, Woodbury MG, Brown JL. The therapeutic effect of functional and transcutaneous electric stimulation on improving gait speed in stroke patients: a meta-analysis. Arch Phys Med Rehabil. 2006;87:853-859.

11. Bogataj U, Gros N, Kljajic M, et al. The rehabilitation of gait in patients with hemiplegia: a comparison between conventional therapy and multichannel functional electrical stimulation therapy. Phys Ther. 1995;75:490-502.

12. Burridge JH, Taylor PN, Hagan SA, et al. The effects of common peroneal stimulation on the effort and speed of walking: a randomized controlled trial with chronic hemiplegic patients. Clin Rehabil. 1997;11:201-210.

13. Alon GRH. Gait and hand function enhancement following training with a multi-segment hybrid-orthosis stimulation system in stroke patients. J Stroke Cerebrovasc Dis. 2003;12:209-216. 
14. Burridge JH, Swain ID, Taylor PN. Functional electrical stimulation: a review of the literature published on common peroneal nerve stimulation for the correction of dropped foot. Rev Clin Gerontol. 1998;8:155-161.

15. Kottink AI, Hermens HJ, Nene AV, et al. A randomized clinical trial of an implantable two-channel peroneal nerve stimulator on walking speed and activity in post stroke hemiplegia. Arch Phys Med Rehabil. 2007;88:971-978.

16. Altman DG, Schulz KF, Moher D, et al. The revised CONSORT statement for reporting randomized trials: explanation and elaboration. Ann Intern Med. 2001;134: 663-694.

17. Holsheimer J, Bultstra G, Verloop AJ, et al. Implantable dual channel peroneal nerve stimulator. In: Proceedings of the Ljubljana FES Conference, Ljubljana, Slovenia. 1933:4244 .

18. van der Aa HE, Bultstra G, Verloop AJ, et al. Application of a dual channel peroneal nerve stimulator in a patient with a "central" drop foot. Acta Neurochir Suppl. 2002;79:105-107.

19. Hermens HJ, Freriks B, Merletti R, et al. European Recommendations for Surface ElectroMyoGraphy: Results of the SENIAM Project. Enschede, the Netherlands: Roessingh Research and Development BV; 1999.

20. Twitchell TE. The restoration of motor function following hemiplegia in man. Brain. 1951;74:443-480.

21. Kottink AI, Oostendorp LJ, Buurke JH, et al. The orthotic effect of functional electrical stimulation on the improvement of walking in stroke patients with a dropped foot: a systematic review. Artif Organs. 2004;28:577-586.

22. Granat MH, Maxwell DJ, Ferguson AC, et al. Peroneal stimulator: evaluation for the correction of spastic drop foot in hemiplegia. Arch Phys Med Rehabil. 1996;77:19-24.

23. Waters RL, McNeal D, Perry J. Experimental correction for footdrop by electrical stimulation of the peroneal nerve. J Bone Joint Surg Am. 1975;57:1047-1054.

24. Brunner E, Domhof S, Langer F. Nonparametric Analysis of Longitudinal Data in Factorial Experiments. New York, NY: Wiley; 2002.

25. Vangeneugden T, Laenen A, Geys H, et al. Applying linear mixed models to estimate reliability in clinical trial data with repeated measurements. Control Clin Trials. 2004;25:13-30.

26. The $\mathrm{R}$ Project for Statistical Computing. Department of Statistics and Mathematics, Wirtschafts Universitat, Wien, Austria. Available at: http://www.r-project.org. Accessed July 10, 2005.

27. Streiner DL, Norman GR. Health Measurement Scales. A Practical Guide to Their Development and Use. Oxford, United Kingdom: Oxford University Press; 1995.

28. Miller GJT, Light KE. Strength training in spastic hemiparesis: should it be avoided? NeuroRehabilitation. 1997;9:17-28. 
29. Rushton DN. Functional electrical stimulation and rehabilitation: an hypothesis. Med Eng Phys. 2003;25:75-78.

30. Zwarts MJ, Stegeman DF. Multichannel surface EMG: basic aspects and clinical utility. Muscle Nerve. 2003;28:1-17.

31. Kallenberg LA, Hermens HJ. Behaviour of motor unit action potential rate, estimated from surface EMG, as a measure of muscle activation level. J Neuroengineering Rehabil. 2006;3:15.

32. Yan T, Hui-Chan CW, Li LS. Functional electrical stimulation improves motor recovery of the lower extremity and walking ability of subjects with first acute stroke: a randomized placebo-controlled trial. Stroke. 2005;36:80-85.

33. Stefanovska A, Gros N, Vodovnik L, et al. Chronic electrical stimulation for the modification of spasticity in hemiplegic patients. Scand J Rehabil Med Suppl. 1988;17:115121.

34. Burridge JH, McLellan DL. Relation between abnormal patterns of muscle activation and response to common peroneal nerve stimulation in hemiplegia. J Neurol Neurosurg Psychiatry. 2000;69:353-361.

\section{Suppliers}

1 Finetech Medical Ltd, 13 Tewin Ct, Welwyn Garden City, Hertfordshire, United Kingdom, AL7 1AU.

2 Tyco Healthcare Deutschland GMBH, Postfach 1217, D-93333, Neustadt/Donau, Germany.

3 Twente Medical Systems International BV, PO Box 6044, 7503 GA, Enschede, the Netherlands.

${ }^{4}$ VICON-UK, 14 Minss Business Park, West Way, Oxford, United Kingdom, OX2 OJB. ${ }^{5}$ SPSS Inc, 233 S Wacker Dr, Chicago, IL 60606. 



\section{Chapter 5}

Effects of an implantable two-channel peroneal nerve stimulator versus conventional walking device on spatiotemporal parameters and kinematics of hemiparetic gait

Anke I.R. Kottink

Martin J.B. Tenniglo

Jaap H. Buurke

Hermie J. Hermens

Submitted 


\begin{abstract}
The objective was to investigate if an implantable two-channel peroneal nerve stimulator can improve gait performance of stroke survivors with a drop foot and whether these improvements are superior to those attained with conventional walking devices, being an ankle-foot orthosis or orthopaedic shoe.

Twenty-three chronic stroke survivors with drop foot fulfilled the predefined inclusion and exclusion criteria and were included in the present randomized controlled trial.

The intervention group received the implantable stimulation system for correction of their drop foot, while the control group continued using their conventional walking device. All subjects were measured on spatiotemporal parameters and hip, knee and ankle kinematics using a three-dimensional video camera system (Vicon $\left.{ }^{\circledR}\right)$ at baseline and at week 26.

Both peroneal nerve stimulator and conventional walking aid seem to have rather comparable effects on the spatiotemporal parameters of the hemiplegic gait. A significant reduction in both ankle plantarflexion angles during swing and at heel strike were found as a result of peroneal nerve stimulation (PNS) in the present study.

The findings of the present study suggest that PNS, in comparison with using a conventional walking aid, seems more effective to provide ankle dorsiflexion during swing and results in a safer initial loading response during stance, which will probably contribute to reducing the risk of stumbling or falling.
\end{abstract}




\section{Introduction}

One of the primary goals in the rehabilitation of a hemiplegic subject is to regain the ability to walk and to develop a safe and efficient gait pattern (1). Hemiparesis due to stroke often results in pretibial muscle weakness. When the weakness is mild, the paretic foot will slap during the loading response. When the dorsiflexion weakness is more severe, foot drop and toe drag will be observed during the swing phase of gait causing the need for a compensated gait pattern and an increased risk of falling. Several strategies are available to compensate for a drop foot, such as circumduction of the ipsilateral leg, increased ipsilateral hip flexion and hip hiking of the contralateral leg. Over time, these compensation strategies can cause secondary complications, for example shortening of the calf muscles, hip or lower back pain, and further deterioration of balance leading to falls and reduced confidence.

Different orthotic devices are used to compensate a drop foot due to upper motor neuron lesion. The most traditional and most often applied devices are the ankle-foot orthoses (AFO) and orthopaedic shoes $(2,3)$. Another treatment option to counteract pretibial muscle weakness is the use of a neuroprosthesis. The first neuroprosthesis for the treatment of a drop foot was introduced by Liberson et al.: the peroneal nerve stimulator (4). In contrast to the more traditional passive walking devices, a neuroprosthesis can strengthen or re-educate muscle function when walking, because when the muscle is being stimulated, it generates all kind of efferent and afferent signals. Both devices support the ankle joint during a different phase of the gait cycle. An AFO provides mediolateral stability at the ankle joint in the stance phase, facilitates toe clearance in swing phase and promotes heel strike (5). A peroneal nerve stimulator stimulates the dorsiflexors that lift the foot in pre-swing and during the swing phase of gait, allowing the foot to clear the ground, and results in a safe initial loading response during stance. There is also evidence that stimulation of the common peroneal nerve may trigger knee and hip flexion (flexion withdraw reflex) and thus facilitate the flexion pattern needed for foot clearance during swing $(6,7)$. According to Merletti et al. (7), this produces a more normal gait pattern and reduces the need for compensatory movements, which implies a more energy efficient use of the hip and knee muscles.

Distance and spatiotemporal gait parameters, such as stride time, step length and duration of foot/floor contact, are useful and fairly easy measures in the assessment of hemiplegic gait (2). The variation in stride time for example is highly correlated with the risk of falls (8). Also lack of symmetry has been associated with poor balance and 
consequently a high risk of falls (9). Spatiotemporal parameters are significantly different in hemiplegic subjects when compared to healthy subjects. In general, hemiplegic subjects walk significantly slower, their rate and length of steps are lower, their swing phase of the paretic limb is shorter, and their stance phase of the nonparetic leg is longer. These differences between the paretic and non-paretic leg make the gait pattern more asymmetrical. Different studies report that the use of an orthosis by hemiplegic subjects significantly improves these spatiotemporal parameters (10-12).

Studies on the effect of drop foot stimulation on the hemiplegic gait pattern and the compensation strategies used by these patients are scarce. A recent intervention study published by Ring et al. (2009) examined the effect of a radio-frequency controlled neuroprosthesis (NESS L300) on gait stability and symmetry and compared the effects obtained with a standard AFO (13). In a group of 15 subjects with chronic hemiparesis, who used the device for a period of 8 weeks, the users of the neuroprosthesis showed better balance control and symmetry during walking when compared with the users of an AFO. However, some of the limitations of their study they mentioned were the relative small sample size, the short period of follow-up and the lack of a baseline measurement with the AFO. In addition, no control group was added to their study. More information about whether peroneal nerve stimulation (PNS) has a superior effect on the hemiplegic gait pattern in comparison with the conventional walking devices would be of great help for clinicians to make better recommendations regarding these technical aids.

The objective of the present randomized controlled trial (RCT) was to investigate if implantable two-channel PNS can improve gait performance of stroke survivors with a drop foot and whether the improvements are superior to those attained with conventional walking devices, being an AFO or orthopaedic shoe. For this purpose, spatiotemporal parameters of both the paretic and non-paretic limb and hip, knee and ankle kinematics of the paretic limb were measured. 


\section{Method}

\section{Study design}

The present study was part of a study that was conducted as a RCT. In this RCT all subjects were assessed 5 times. The baseline measurement took place about 1 week before the randomized procedure, and the follow-up measurements were performed, 4 , 8,12 and 26 weeks after implanting the two-channel peroneal nerve stimulator in the intervention group. Subjects assigned to the control group were measured in the same weeks as subjects assigned to the intervention group. All assessments were performed by the same experienced examiners (AK, MT). Three weeks after the implantation procedure subjects received the transmitter and instructions were given on the proper use of the peroneal nerve stimulator. In order to assess the effect of an implantable twochannel peroneal nerve stimulator versus a conventional walking aid on the gait pattern of chronic hemiparetic subjects, measurements were performed at baseline and at week 26. Blinding of both the study personnel and participants was not possible due to the surgical procedure. All data were analyzed according to an intention-to-treat principle.

\section{Subjects}

Most subjects were recruited in response to an article in a local Dutch newspaper describing the first results on the implantable stimulator in stroke survivors. Some subjects were recruited through consultant and general practitioner referrals and in some cases on the advice of the physiotherapist treating the subject. Both recruitment strategies resulted in a selected sample of well motivated stroke survivors. Because of the invasive nature of the treatment, selection of the most appropriate subjects is very important. Therefore subjects that were most likely to experience treatment success were selected. Table 5.1 shows the criteria for inclusion and exclusion that were used in the trial.

Subjects who fulfilled the selection criteria were admitted to the trial. One week after the baseline assessment, they were randomly allocated to either the intervention group or the control group. Random allocation was performed in blocks of two subjects, to ensure a close balance of the numbers in each group. The randomisation procedure was performed by a independent individual. Each time two suitable subjects were included in the study, their names were given to the person who was responsible for the randomization procedure. This person informed the examiner, after the baseline 
measurement was performed, which subject was allocated to the intervention group and which subject was allocated to the control group.

Table 5.1 Selection criteria

\begin{tabular}{ll}
\hline Inclusion criteria & Exclusion criteria \\
\hline $\begin{array}{l}\text { Drop foot identified by an inability to achieve a } \\
\text { normal heel strike during walking }\end{array}$ & Age $<18$ year \\
$\begin{array}{l}\text { First hemiplegia of at least } 6 \text { months duration as a } \\
\text { result of a CVA with a stable neurology } \\
\text { Subject is an outdoor walker }\end{array}$ & $\begin{array}{l}\text { Passive dorsiflexion of the ankle }<5 \text { o with knee in } \\
\text { extension } \\
\text { Medical conditions other than CVA, i.e. neurological, } \\
\text { rheumatic, cardio-vascular or systemic disorders } \\
\text { (including Diabetes Mellitus) limiting the function of } \\
\text { walking } \\
\text { Injury to deep and superficial peroneal nerve and } \\
\text { sciatic nerve } \\
\text { Any medical condition that would exclude the use of } \\
\text { able to give an informed consent } \\
\text { atgical procedure or anesthetic } \\
\text { Not able to don and doff the equipment } \\
\text { Pregnancy }\end{array}$ \\
\hline
\end{tabular}

The experiment protocol was approved by the local Medical ethics committee and the subjects gave their informed consent before participating in the experiments.

\section{Stimulation system}

The implantable two-channel peroneal nerve stimulator was manufactured by Finetech Medical Limited ${ }^{1}$ and consists of an external transmitter with a built in antenna, a foot switch, and an implantable part consisting of the stimulator, the two leads and the bipolar intra-neural electrodes $(14,15)$.

The transmitter weights approximately $0.1 \mathrm{~kg}$ and is attached with a strap on the lateral side of the lower leg, over the site of the implant, just below the knee. A footswitch placed under the heel of the subject's foot inside the shoe determines the on-and-off switching of the stimulation. Over night, the battery of the transmitter is charged.

The implantable part is a passive device, receiving information carried by the radio frequency signals and converting that information into the stimulation pulses of the desired amplitude and frequency. One electrode is placed below the epineurium of the superficial peroneal nerve (eversion) and one below the epineurium of the deep peroneal nerve (dorsiflexion). For a more detailed description of the implantable stimulator see Kottink et al. (16). 


\section{Assessment of spatiotemporal parameters and kinematics}

Both the baseline and follow-up assessment were performed in the gait laboratory of Roessingh Research and Development (RRD). The experiment started with collecting the anthropometric data of the subjects. The gait pattern was recorded by using a threedimensional video camera system (Vicon ${ }^{\circledR}$, version 370 ). The model used in the present study was based on the Davis-Kadaba model (10;17-19). In total 17 markers were placed on both legs and pelvis (first and fifth head of the metatarsal, lateral malleolus of the ankle, heel, lateral epicondyle of the femur, anterior superior iliac spine, sacrum and four reflective markers on the lateral aspect of the upper and lower legs). During the static measurement extra markers were placed on the medial epicondyle of the femur and the medial malleolus of the ankle on both legs. The subjects walked at their natural speed across a field of view of 6 stationary cameras. When possible, subjects were instructed to walk without walking stick. If this was not the case, this was recorded and three additional markers were placed on the walking stick. The condition during baseline was the standard for the follow-up measurement. To exclude the influence of acceleration and deceleration at the beginning and end of the walkway, subjects started walking far before the field of view of the cameras. Four trials were analysed and averaged for each subject to obtain all kinematic parameters. All markers were labelled by means of the Vicon Bodybuilder program version 3.55 and the gait data were analysed with the Vicon model output viewer. First the gait data were normalised to a $0-100 \%$ gait cycle and then averaged. Spatiotemporal gait parameters of both the paretic and non-paretic limb, such as stride time, stride length, stride width, step length, duration of stance phase, duration of the first and second double and single support phases and walking speed were measured. Also the hip-, knee and ankle kinematics of the paretic leg were measured by the Vicon ${ }^{\circledR}$ system. Changes during movement were quantified by comparing calculated angles at predefined points in the gait phases of interest. These phases were based on manually placed events and their definitions are shown in table 5.2 .

Table 5.2 Definitions of gait phases

\begin{tabular}{ll}
\hline Phase & Description \\
\hline Pre swing & Opposite heel strike - heel off \\
Swing & Heel off - heel strike \\
Initial swing & First $30 \%$ of the swing phase \\
Mid swing & $30-70 \%$ of the swing phase \\
Terminal swing & $70-100 \%$ of the swing phase \\
\hline
\end{tabular}




\section{Statistical analysis}

An Analysis of Covariance (ANCOVA) was used to test if differences in all spatiotemporal parameters and kinematics were present between the intervention and control group at week 26. To correct for possible differences at baseline between the intervention and control group, the baseline scores were taken as covariate. Group was taken as only fixed factor in the analysis. Normality plots of the residuals were made to examine if the data were distributed according to a normal distribution. The statistical analyses was performed with SPSS version 11.5 for Windows. The significance level $\alpha$ was set at .05 . 


\section{Results \\ Subjects}

Figure 5.1 shows the flow of participant throughout the RCT. In total, 29 subjects with drop foot due to stroke participated in the RCT, of which 14 subjects were allocated to the intervention group and 15 subjects were allocated to the control group. Both the orthotic and therapeutic effect of the implantable 2-channel peroneal nerve stimulator on walking speed and some other parameters were previously described $(20,21)$. Since the goal of the current study was to examine if there is a surplus value of implantable PNS over the conventional treatment of a drop foot, this study is based on a subset of those subjects. Since 4 subjects of the intervention group did not use a conventional walking aid to correct their drop foot, they were excluded from analysis in the present study. In the past these subjects were all advised by their therapist/doctor to use a conventional walking aid, but at the moment of inclusion they did not use it anymore. Two subjects of the control group were excluded since their gait pattern was not recorded at baseline. Table 5.3 shows the characteristics of the included participants of the present study. Subject characteristics were not significantly different between both groups, with the exception of mean time after stroke, which was longer for the intervention group.

In the intervention group $(\mathrm{n}=10), 7$ subjects used an AFO and 3 subjects wore orthopaedic shoes. Four subjects used a polypropylene, non-articulated AFO with two crossed posterior steels and an open heel (Ottobock, Otto Bock Benelux bv, Son en Breugel, The Netherlands's), 2 subjects used a custom made polypropylene, nonarticulated AFO with a large posterior steel (Camp, Basko Healthcare, Zaandam, The Netherlands ${ }^{3}$ ) and 1 subject used a polypropylene, non-articulated AFO with a small posterior steel (Dynafo, Ortho-Medico, Herzele, Belgium ${ }^{4}$ ). In the control group ( $\left.\mathrm{n}=13\right)$, all subjects wore an AFO. Three subjects used a polypropylene, non-articulated AFO with two crossed posterior steels and an open heel, 4 subjects used a custom made polypropylene, non-articulated AFO with a large posterior steel, 3 subjects used a polypropylene, non-articulated AFO with a small posterior steel and 1 subject used a non-custom made polypropylene, non-articulated AFO with a large posterior steel. Two subjects dropped out of the study, 1 woman in the intervention and 1 men in the control group. The implant of the subject in the intervention group who dropped out failed after functioning properly for about 10 weeks. An investigation of the explanted 
system showed that the failure was caused by a technical defect in the epineural electrode responsible for the dorsiflexion movement.

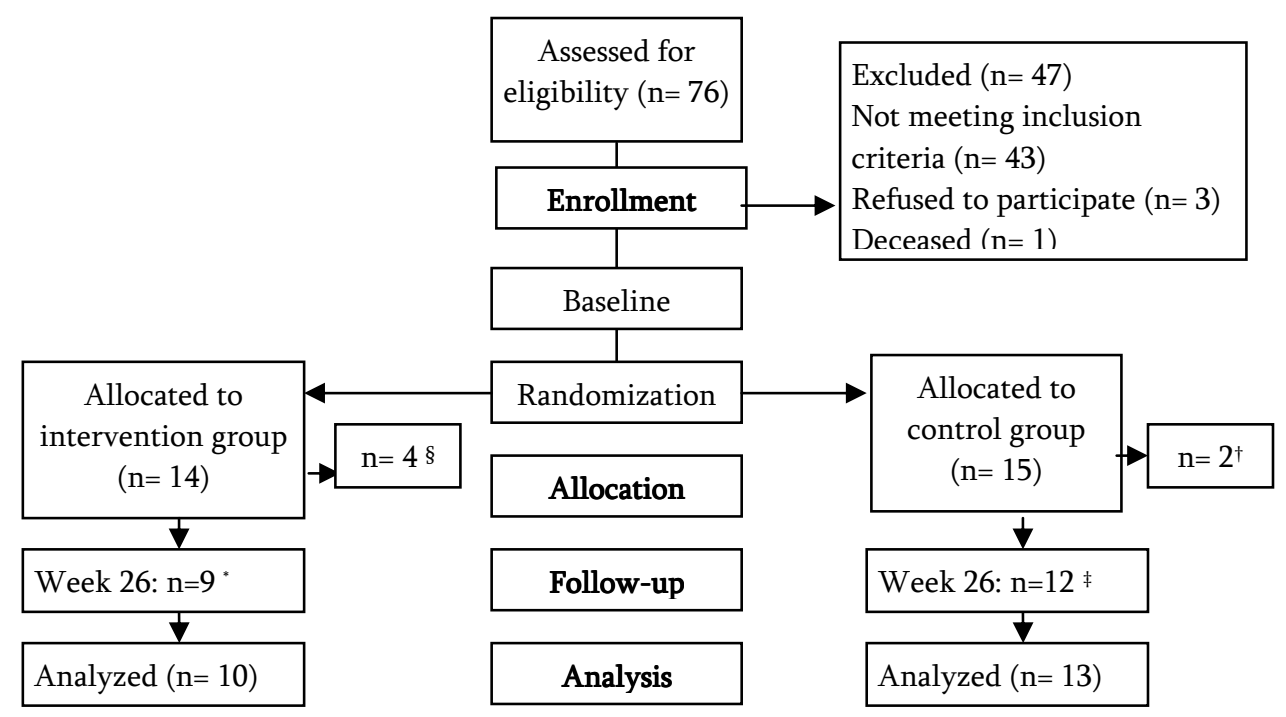

Figure 5.1 Flowchart of patients through the study

$\S$ No conventional walking device

${ }^{\dagger}$ No gait pattern recorded

* Defective implant

‡ Psychologic issues not related to the study

One subject in the control group dropped out in week 11, because of psychologic issues not related to the study. The remaining 9 subjects in the intervention group didn't report any technical failure of the stimulation system and continued to use the stimulator during the entire follow-up period.

\section{Spatiotemporal parameters}

No difference in walking speed was found between both study groups, so no influence of this parameters is expected on the present findings. Table 5.4 shows the spatiotemporal parameters of both the paretic and non-paretic limb for both the intervention and control group. 
Table 5.3 Subject characteristics

\begin{tabular}{lcc}
\hline & Intervention group & Control group \\
\hline Number of subjects (drop outs) & $10(1)$ & $13(1)$ \\
Men/ Women & $7 / 3$ & $8 / 5$ \\
Mean age (sd) & 55.6 years $(13.16)$ & 53.31 years $(10.55)$ \\
Mean time after stroke (sd) & 9 years $(10.04)$ & 6.15 years $(4.81)$ \\
Left side affected & 6 & 8 \\
Right side affected & 4 & 5 \\
\hline
\end{tabular}

Table 5.4 Spatiotemporal parameters (mean (sd))

\begin{tabular}{|c|c|c|c|c|c|}
\hline & \multicolumn{2}{|c|}{ Intervention } & \multicolumn{2}{|c|}{ Control } & \multirow[t]{2}{*}{$\mathbf{P}$} \\
\hline & Baseline & Week 26 & Baseline & Week 26 & \\
\hline Walking speed $(\mathrm{m} / \mathrm{sec})$ & $0.82(0.18)$ & $0.92(0.17)$ & $0.73(0.2)$ & $0.78(0.23)$ & .55 \\
\hline Stride time (sec) & $1.4(0.3)$ & $1.3(0.1)$ & $1.4(0.3)$ & $1.4(0.3)$ & .99 \\
\hline Stride length $(\mathrm{cm})$ & $110(12.8)$ & $113(14.6)$ & $100(16.9)$ & $103(20.8)$ & .30 \\
\hline Stride width $(\mathrm{cm})$ & $28(5.6)$ & $27(5.6)$ & $29(7.1)$ & $26(5.9)$ & .58 \\
\hline \multicolumn{6}{|l|}{ Step length (cm) } \\
\hline Paretic side & $54(10.3)$ & $55(13.4)$ & $53(10)$ & $56(13.8)$ & .29 \\
\hline Non-paretic side & $59(8)$ & $60(9.5)$ & $53(8.3)$ & $55(11.7)$ & .06 \\
\hline \multicolumn{6}{|l|}{ Duration stance phase (\%) } \\
\hline Paretic side & $58.9(3.4)$ & $55.9(3)$ & $60.9(2.8)$ & $60.6(3.2)$ & .04 \\
\hline Non-paretic side & $68.5(3.8)$ & $66.5(2)$ & $68.7(5)$ & $69.3(5)$ & .06 \\
\hline \multicolumn{6}{|l|}{$1^{\text {st }}$ double support phase (\%) } \\
\hline Paretic side & $10.5(2)$ & $8.2(1.6)$ & $12.8(2.9)$ & $12.6(4)$ & .07 \\
\hline Non-paretic side & $17.1(4.4)$ & $14.2(2.5)$ & $16(4.1)$ & $16.4(4.3)$ & .1 \\
\hline \multicolumn{6}{|c|}{$2^{\text {nd }}$ double support phase (\%) } \\
\hline Paretic side & $17.1(4.8)$ & $14.3(2.3)$ & $16.8(4.3)$ & $16.3(3.4)$ & .1 \\
\hline Non-paretic side & $11.1(1.6)$ & $8.7(1.4)$ & $13.1(3)$ & $13.4(3.4)$ & .01 \\
\hline \multicolumn{6}{|l|}{$1^{\text {st }}$ single support phase (\%) } \\
\hline Paretic side & $31.3(3.9)$ & $33.5(2.2)$ & $31.3(4.9)$ & $31.7(5.3)$ & .3 \\
\hline Non-paretic side & $40.4(3)$ & $43.6(3)$ & $39.5(2.6)$ & $39.5(3.5)$ & .03 \\
\hline
\end{tabular}

Paretic limb. The duration of the stance phase changed significantly between both group. In the intervention group a reduction in the duration of the stance phase from 58.9 to 55.9 \% was found, while the control group did not show a change (61\%). The remaining spatiotemporal parameters did not change significantly between both groups.

Non-paretic limb. The duration of the second double and single support phase changed significantly between both groups. In the intervention group a shorter period of time in double support and a longer period of time in single support was found, while the control group did not show a change in both parameters. The remaining spatiotemporal parameters did not change significantly between both groups. 

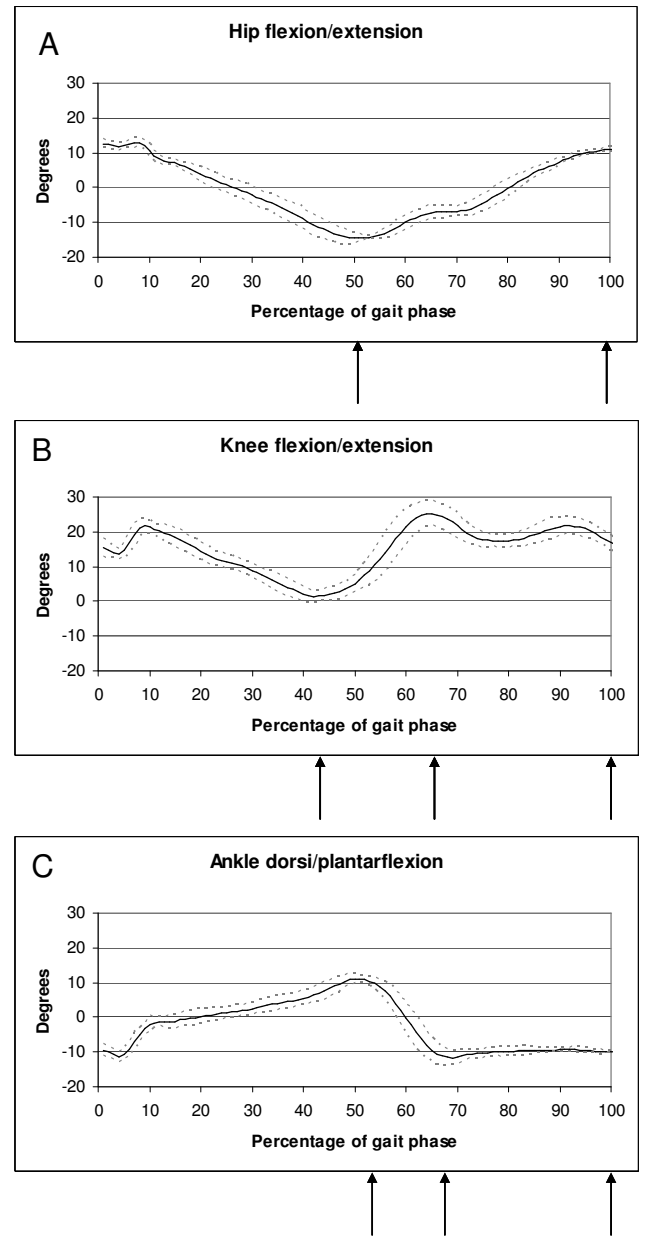

Figure 5.2 A typical example of the kinematics in the sagittal plane of a subject with left hemiplegia: hip flexion/extension (A), knee flexion/extension (B) and ankle dorsi/plantarflexion (C)

The bands represent the average \pm one sd. The arrows reflect the joint angles taken in the analysis.

\section{Kinematics}

Figure 5.2 shows a typical example of the hip, knee and ankle kinematics in the sagittal plane of a subject with left hemiplegia. The kinematics of the paretic limb for both the intervention and control group are shown in table 5.5. A significant reduction of $5^{\circ}$ in ankle plantarflexion was found in the intervention group, while using their implantable stimulation device, compared with the control group who showed a small increase of $2^{\circ}$ in plantarflexion. With regard to the ankle plantarflexion angle at heel strike, a significant reduction of $7^{\circ}$ was found in the intervention group, resulting in a 
plantarflexion angle of $0^{\circ}$ during walking with their implantable stimulation device, compared with the control group, whose plantarflexion angle did not change during the trial $\left(9^{\circ}\right)$. The remaining joint angular parameters did not change significantly between both groups.

Table 5.5 Kinematics of paretic limb (mean (sd))

\begin{tabular}{|c|c|c|c|c|c|}
\hline & \multicolumn{2}{|c|}{ Intervention } & \multicolumn{2}{|c|}{ Control } & \multirow[t]{2}{*}{$\mathbf{P}$} \\
\hline & Baseline & Week 26 & Baseline & Week 26 & \\
\hline \multirow[t]{2}{*}{ Hip $\mathrm{ROM}\left({ }^{\circ}\right)$} & 31.24 & 31.93 & 33.51 & 37.14 & .19 \\
\hline & $(7.48)$ & $(9.23)$ & $(9.28)$ & $(8.66)$ & \\
\hline \multirow[t]{2}{*}{ Minimum hip extension angle $\left({ }^{\circ}\right)$} & -7.08 & -7.27 & -8.56 & -12.26 & .33 \\
\hline & $(5.75)$ & $(6.06)$ & $(8.96)$ & $(9.51)$ & \\
\hline \multirow[t]{2}{*}{ Maximum hip flexion angle $\left({ }^{\circ}\right)$} & 24.16 & 24.66 & 24.95 & 24.88 & .8 \\
\hline & $(8.05)$ & $(6.74)$ & $(8.02)$ & $(10.37)$ & \\
\hline \multirow[t]{2}{*}{ Knee ROM $\left(^{\circ}\right)$} & 27.65 & 26.5 & 40.03 & 44.04 & .12 \\
\hline & $(10.68)$ & $(13.96)$ & $(13.95)$ & $(15.49)$ & \\
\hline \multirow[t]{2}{*}{ Minimum knee flexion/extension angle $\left(^{\circ}\right)$} & 4.03 & 6.32 & 0.21 & -2.83 & .04 \\
\hline & $(7.91)$ & $(9.21)$ & $(6.32)$ & $(6.6)$ & \\
\hline \multirow[t]{2}{*}{ Maximum knee flexion angle $\left({ }^{\circ}\right)$} & 31.68 & 32.82 & 40.25 & 41.21 & .53 \\
\hline & $(10.49)$ & $(11.48)$ & $(13.48)$ & $(14.18)$ & \\
\hline \multirow[t]{2}{*}{ Ankle ROM $\left(^{\circ}\right)$} & 18.56 & 13.34 & 14.4 & 16.14 & .03 \\
\hline & (7.64) & $(4.76)$ & (7.93) & $(5.95)$ & \\
\hline \multirow[t]{2}{*}{ Maximum ankle dorsiflexion angle $\left(^{\circ}\right)$} & 8.32 & 8.52 & 3.08 & 4.44 & .84 \\
\hline & (6.32) & $(6.41)$ & $(6.6)$ & $(6.04)$ & \\
\hline \multirow[t]{2}{*}{ Minimum ankle plantarflexion angle $\left(^{\circ}\right)$} & -10.23 & -4.82 & -11.32 & -11.7 & .03 \\
\hline & $(6.77)$ & $(5.56)$ & $(3.96)$ & $(6.35)$ & \\
\hline \multirow{2}{*}{ Knee flexion at heel strike $\left(^{\circ}\right)$} & 13.5 & 17.27 & 12.26 & 9.98 & .08 \\
\hline & $(6.69)$ & $(6.2)$ & $(5.66)$ & $(8.58)$ & \\
\hline \multirow[t]{2}{*}{ Ankle plantarflexion at heel strike $\left(^{\circ}\right)$} & -6.85 & 0.03 & -8.71 & -8.9 & .01 \\
\hline & $(5.71)$ & $(4.52)$ & $(4.04)$ & (6.11) & \\
\hline
\end{tabular}




\section{Discussion}

To examine whether a superior value of FES was present, a comparison was made between the effect of an implantable peroneal nerve stimulator versus the conventional treatment on the gait pattern of chronic stroke survivors.

Subjects who used implantable PNS showed a reduction in the duration of the stance phase of the paretic limb. On the non-paretic side a shorter period of time in double support and a longer period of time in single support was found. All spatiotemporal parameters in the control group remained constant during the trial period. These changes were quit small; probably because of the small standard deviations the level of significance was reached. Therefore these findings are not expected to influence the gait performance of subjects walking with PNS in a functionally relevant way. Literature about the effect of a drop foot neuroprosthesis on the gait pattern of stroke subjects is scarce; most studies focused on outcome measures like walking speed (22). Recently, one uncontrolled study examined the effect of a transcutaneous neuroprosthesis for drop foot in comparison with an AFO on postural control during walking (13). They reported a shorter stride time, a less asymmetrical gait pattern and a less variable and more consistent single limb support of the paretic leg after 8 weeks use of the neuroprosthesis. These results are not in agreement with the current findings. In incomplete spinal cord injured (SCI) subjects, Ladouceur et al. found minor effects of FES-assisted walking on spatiotemporal parameters, such as stride length, stride frequency, stance and swing duration (23).

The influence of an orthosis on spatiotemporal parameters of stroke subjects has been described more often in literature (12,24,25). Franceschini et al. (2003) found that an orthosis results in a significant reduction in the duration of the stride cycle, the duration of the stance phase and the duration of the double support of both limbs, when compared to walking without orthosis (24). These results are in agreement with results found by Gök et al. (2003), who reported positive effects on hemiplegic gait parameters as a result of three different AFOs, expressed as an increase in cadence, single and double step length, ankle dorsiflexion angle at heel strike and swing of the paretic limb (25). Also Wang et al. (2007) assessed the change in balance performance and the improvement in gait performance of subjects with hemiparesis as a result of wearing an AFO (12). They found that the step length of the non-paretic leg increased and that the maximal excursion toward the paretic leg improved as a result of wearing an AFO. 
Compared to healthy controls hemiplegic subjects usually have less dorsiflexion during mid-swing and heel strike due to loss of motor control, spasticity of the gastrocnemiussoleus group and ankle contracture (25). In healthy subjects, the increase in pretibial muscle action during terminal swing assures that the ankle and foot are adequately prepositioned for initial contact (26). Regarding the kinematics of the paretic limb, a significant reduction in both ankle plantarflexion angles during swing and at heel strike were found as a result of PNS in the present study. Therefore stimulation of the pretibial muscles by an implanted FES system seems very effective to provide ankle dorsiflexion during the swing phase of gait and to result in a safe initial contact and loading response in the stance phase. This adequate pre-positioning is likely to prevent stroke patients from stumbling or falling. This was confirmed by Kim et al., who compared the effect of FES with that of a hinged AFO in subjects with an incomplete SCI. They found that foot clearance was improved with FES and not with an AFO (27). Ladouceur et al. reported also that the ankle plantarflexion angle at foot contact decreased by $5.6^{\circ}$ when the FES system was used in incomplete SCI subjects (23). In patients with Multiple Sclerosis, Sheffler et al. found that PNS significantly increased ankle dorsiflexion angle at initial contact compared to an AFO (28). These findings are consistent with the results of the present study.

No evidence was found in the present study that PNS may trigger hip and knee flexion, which would further facilitate the flexion pattern needed for foot clearance during swing. The hip and knee angles found in the intervention group during walking with a peroneal nerve stimulator were comparable with the angles shown during walking with a conventional walking aid at baseline. In the past, Burridge et al. described that also a reflex withdrawal response is triggered by PNS, which is characterised by knee flexion with slight flexion, external rotation and abduction of the hip by activity of the hamstring muscles (6). Also Merletti et al. reported that walking with FES implies a more efficient use of the hip and knee muscles by avoiding the need for compensatory movements (7). However, both studies did not measure this parameter in their study, they only mentioned this assumption to explain the results found in their study.

\section{Limitations}

A limitation of this study may be the generalization of the results to the broader population with stroke. Patients included in the present trial were primarily selected on 
their suitability for implantable FES, which means that a subpopulation of less affected stroke subjects was included.

Furthermore, participants and investigators were not blinded. Participant blinding was not possible and investigators blinding was not possible because the peroneal nerve stimulator and the conventional walking aid could not be hidden from view during the measurement. To minimize this bias, the involvement of the investigators (eg, instructions, encouragement) was kept consistent during data collection and the data processing was blinded.

\section{Conclusion}

The findings of the present study suggest that PNS, in comparison with using a conventional walking aid, seems more effective to provide ankle dorsiflexion during swing and results in a safer initial loading response during stance, which is likely to reduce the risk of stumbling or falling in chronic stroke survivors with a drop foot. No evidence was found that PNS may trigger hip and knee flexion. On the remaining spatiotemporal parameters and kinematics of the hemiplegic gait a peroneal nerve stimulator and a conventional walking aid seem to have a comparable effect. These results support the use of PNS as treatment option for a drop foot in chronic stroke survivors. 


\section{References}

1. Bohannon RW, Horton MG, Wikholm JB. Importance of four variables of walking to patients with stroke. Int J Rehabil Res 1991;14(3):246-50.

2. Hale S WJ. The effects of different ankle-foot orthoses on the kinematics of hemiplegic gait. Orthotics and Prosthetics 1987;41(3):40-9.

3. Geboers JF, Drost MR, Spaans F, Kuipers H, Seelen HA. Immediate and long-term effects of ankle-foot orthosis on muscle activity during walking: a randomized study of patients with unilateral foot drop. Arch Phys Med Rehabil 2002 Feb;83(2):240-5.

4. Liberson WT, Holmquest HJ, Scot D, Dow M. Functional electrotherapy: stimulation of the peroneal nerve synchronized with the swing phase of the gait of hemiplegic patients. Arch Phys Med Rehabil 1961 Feb;42:101-5.

5. Leung J MA. Impact of ankle-foot orthosis on gait and leg muscle activity in adults with hemiplegia: systematic literature review. Physiotherapy 2003;89:39-55.

6. Burridge J, Taylor P, Hagan S, Wood D, Swain I. The effect of common peroneal nerve stimulation on quadriceps spasticity in hemiplegia. Physiotherapy 1997;83:82-9.

7. Merletti R, Andina A, Galante M, Furlan I. Clinical experience of electronic peroneal stimulators in 50 hemiparetic patients. Scand J Rehabil Med 1979;11(3):111-21.

8. Hausdorff JM, Ring H. Effects of a new radio frequency-controlled neuroprosthesis on gait symmetry and rhythmicity in patients with chronic hemiparesis. Am J Phys Med Rehabil 2008 Jan;87(1):4-13.

9. Yogev G, Plotnik M, Peretz C, Giladi N, Hausdorff JM. Gait asymmetry in patients with Parkinson's disease and elderly fallers: when does the bilateral coordination of gait require attention? Exp Brain Res 2007 Mar;177(3):336-46.

10. Kadaba MP, Ramakrishnan HK, Wooten ME, Gainey J, Gorton G, Cochran GVB. Repeatability of kinematic, kinetics and electromyographic data in normal adult gait. Journal of Orthopaedic Research 1989;7:849-60 .

11. Pohl M, Mehrholz J. Immediate effects of an individually designed functional ankle-foot orthosis on stance and gait in hemiparetic patients. Clin Rehabil 2006 Apr;20(4):324-30.

12. Wang RY, Lin PY, Lee CC, Yang YR. Gait and balance performance improvements attributable to ankle-foot orthosis in subjects with hemiparesis. Am J Phys Med Rehabil 2007 Jul;86(7):556-62.

13. Ring H, Treger I, Gruendlinger L, Hausdorff JM. Neuroprosthesis for footdrop compared with an ankle-foot orthosis: effects on postural control during walking. J Stroke Cerebrovasc Dis 2009 Jan;18(1):41-7.

14. Holsheimer J, Bultstra G, Verloop AJ, van der Aa HE, Hermens HJ. Implantable dual channel peroneal nerve stimulator. Proceedings Ljubljana FES Conference, Ljubljana, Slovenia 1993;42-4. 
15. van der Aa HE, Bultstra G, Verloop AJ, Kenney L, Holsheimer J, Nene A, Hermens HJ, Zilvold G, Buschman HP. Application of a dual channel peroneal nerve stimulator in a patient with a "central" drop foot. Acta Neurochir Suppl 2002;79:105-7.

16. Kottink AI, Hermens HJ, Nene AV, Tenniglo MJ, van der Aa HE, Buschman HP, Ijzerman MJ. A randomized controlled trial of an implantable 2-channel peroneal nerve stimulator on walking speed and activity in poststroke hemiplegia. Arch Phys Med Rehabil 2007 Aug;88(8):971-8.

17. Davis R, Ounpuu S, Tyburski D, Gage J. A gait analysis data collection and reduction technique. Human Movement Sciences 1991;10:575-87.

18. Kadaba MP, Ramakrishnan HK, Wootten ME, Stein JL. Lower extremity joint moments and ground reaction torque in adult gait. Biomechanics of Normal and Prosthetic Gait. American Society of Mechanical Engineers 1987;7:87-92.

19. Kadaba MP, Ramakrishnan HK, Wooten ME. Lower extremity kinematics during level walking. Journal of Orthopaedic Research 1990;8:849-60.

20. Kottink AI, Hermens HJ, Nene AV, Tenniglo MJ, van der Aa HE, Buschman HP, Ijzerman MJ. A randomized controlled trial of an implantable 2-channel peroneal nerve stimulator on walking speed and activity in poststroke hemiplegia. Arch Phys Med Rehabil 2007 Aug;88(8):971-8.

21. Kottink AI, Hermens HJ, Nene AV, Tenniglo MJ, Groothuis-Oudshoorn CG, IJzerman MJ. Therapeutic effect of an implantable peroneal nerve stimulator in subjects with chronic stroke and footdrop: a randomized controlled trial. Phys Ther 2008 Apr;88(4):437-48.

22. Kottink AI, Oostendorp LJ, Buurke JH, Nene AV, Hermens HJ, IJzerman MJ. The orthotic effect of functional electrical stimulation on the improvement of walking in stroke patients with a dropped foot: a systematic review. Artif Organs 2004 Jun;28(6):577-86.

23. Ladouceur M, Barbeau H. Functional electrical stimulation-assisted walking for persons with incomplete spinal injuries: changes in the kinematics and physiological cost of overground walking. Scand J Rehabil Med 2000 Jun;32(2):72-9.

24. Franceschini M, Massucci M, Ferrari L, Agosti M, Paroli C. Effects of an ankle-foot orthosis on spatiotemporal parameters and energy cost of hemiparetic gait. Clin Rehabil 2003 Jul;17(4):368-72.

25. Gok H, Kucukdeveci A, Altinkaynak H, Yavuzer G, Ergin S. Effects of ankle-foot orthoses on hemiparetic gait. Clin Rehabil 2003 Mar;17(2):137-9.

26. Jacqueline Perry. Gait analysis. Normal and pathological function. SLACK Incorporated; 1992.

27. Kim CM, Eng JJ, Whittaker MW. Effects of a simple functional electric system and/or a hinged ankle-foot orthosis on walking in persons with incomplete spinal cord injury. Arch Phys Med Rehabil 2004 Oct;85(10):1718-23. 
28. Sheffler LR, Bailey SN, Chae J. Spatiotemporal and kinematic effect of peroneal nerve stimulation versus an ankle-foot orthosis in patients with multiple sclerosis: a case series. PM R 2009 Jul;1(7):604-11.

\section{Suppliers}

${ }^{1}$ Finetech Medical Limited (implantable two-channel peroneal nerve stimulator), 13 Tewin Court, Welwyn Garden City, Hertfordshire, AL7 1AU, England

${ }^{2}$ Otto Bock Benelux B.V. (Ottobock AFO), P.O. Box 133, 5690 AC Son en Beugel, The Netherlands

${ }^{3}$ Basko Healthcare (custom made and non-custom made Camp AFO), P.O. Box 2194, 1500 GD Zaandam, The Netherlands

${ }^{4}$ Ortho-MEDICO (Dynafo AFO), Mutsaardstraat 47, B-9550 Herzele, Belgium 



\title{
Chapter 6
}

Measuring quality of life in stroke subjects receiving an implanted neural prosthesis for drop foot

\author{
Anke I.R. Kottink \\ Catharina G. Groothuis-Oudshoorn \\ Maarten J. IJzerman \\ Hermie J. Hermens
}

Accepted by Artificial Organs

Reprinted with permission 


\section{Abstract}

The aim was to determine if the treatment of a drop foot by means of an implantable two-channel peroneal nerve stimulator improves health-related quality of life (HRQoL). All subjects were measured at baseline and after a follow-up period of 12 and 26 weeks. Twenty-nine stroke survivors with chronic hemiplegia with drop foot who fulfilled the predefined inclusion and exclusion criteria were included in the present randomized controlled trial. The intervention group received an implantable twochannel peroneal nerve stimulator for correction of their drop foot. The control group continued using their conventional walking device, consisting of an ankle foot orthosis, orthopedic shoes, or no device.

HRQoL was assessed in two different ways: (i) by taking descriptive measures, that is, the Short Form-36 (SF-36; generic measure) and the Disability Impact Profile (DIP; specific measure); and (ii) by obtaining preference-based utilities both measured with the time trade-off (direct way) and by calculating them from the EuroQol (EQ-5D) and SF-36.

A significant positive effect of the implantable device was found on the physical functioning domain, the general health domain, and the physical component summary score of the SF- 36. For the DIP, a significant improvement was found on the domains mobility, self-care, and psychological status in the intervention group. Regarding the preference-based utility measures, a significant effect was found for the utility index calculated from the EQ-5D.

The implantable two-channel peroneal nerve stimulator seems to be efficient to improve HRQoL, mainly the domains related to physical functioning. A relation was present between the utility indexes calculated from the EQ-5D and SF-36. 


\section{Introduction}

The incidence of stroke increases with age, and is an important cause of mortality and morbidity in the western world. The ultimate goal in the rehabilitation of stroke survivors is to regain independence and improve quality of life (QoL) (1). Gotay et al. (2) defined QoL as a state of well-being which is a composite of two components, that is, (i) the patients ability to perform everyday activities which reflect physical, psychological, and social well-being and (ii) patient satisfaction with levels of functioning and the control of disease and/or treatment-related symptoms. So, from a patients' perspective, QoL can be seen as a result of a complex process of interaction between personal characteristics, medical outcome, coping behavior, social support, and the quality of received health care (1).

In the past, several studies examined QoL in longterm stroke survivors and identified variables that predict QoL after stroke (2-7). From these studies, it became clear that a substantial proportion of stroke survivors have very poor QoL and that QoL in only a small portion of subjects is restored to a prestroke level. Besides depression, cognitive and physical functioning proved also to be important factors for life satisfaction in terms of health-related QoL (HRQoL) (7), one aspect or domain of QoL. Persisting motor impairment and disability on the performance of activities of daily living seemed to have a negative effect on several aspects of life satisfaction, as assessed by different QoL scales (3-5,8,9).

Recently, some studies were published which evaluated the impact of a mobility intervention or therapy on QoL in stroke survivors (10-12). Fernandes et al. (10) evaluated QoL with the 36-item short form of the Medical Outcomes Study questionnaire (SF-36) before and after the treatment with a functional electric orthosis on the paretic leg for the rehabilitation of walking in a group of 50 stroke subjects. They reported that the average values in all domains of SF-36 improved significantly as a result of the orthosis, especially the domain physical functioning. Rydwik et al. (11) and Langhammer et al. (12) examined the effect of exercises of the affected foot and physiotherapy exercise regimes on HRQoL, respectively. Rydwik et al. did not find a significant difference in HRQoL measured by the SF-36 between the intervention and control group, while Langhammer et al. found that regular exercise with self-initiated training seemed to enhance $H$ RQoL more than intensive exercise with scheduled intensive training. In addition, the degree of motor function, balance, walking capacity, 
and independence in the performance of activities of daily living is of importance for perceived HRQoL.

In literature, different instruments exist to measure HRQoL. There is broad consensus that the assessment of QoL should include aspects of physical, psychological, social, and general health, which makes it necessary to use several measures of HRQoL (13). Measures of HRQoL can be divided into two categories: descriptive and preferencebased utility measures $(14,15)$. Descriptive measures result in an index (generic measures, i.e., the EuroQol (EQ-5D) (16) and the SF-36 (17) or a profile (disease specific measures, i.e., Nottingham health profile and Disability Impact Profile (DIP)) $(16,18)$. Although many of these descriptive instruments include items about a subjects' overall life satisfaction, none is primarily concerned with the quantification of the preferences of a subject regarding health status. Preference-based utility measures were developed to address the significance of health impairments in a systematic way (14). Utility scores are obtained to calculate quality adjusted life-years (QALYs), being an outcome measure that reflects both life expectancy and quality of life and which is now widely used in medical decision making and health economics $(19,20)$. As such, QALYs provide a common metric for comparisons across interventions and disease areas (21).

Preference-based utilities may be measured in a direct way or an indirect way (16). One of the most common techniques to measure utilities in a direct way is the time trade-off (TTO), in which respondents are asked how many months or years of life they would be willing to give up to be in a better health state (22). Scores can range from 0 to 1.0 , where 0 represents death and 1.0 represents perfect health. By means of the EQ-5D and the SF-36, measures which are widely used for the measurement of HRQoL in stroke subjects, utilities can be obtained in an indirect way. So, descriptive and preferencebased measures provide different but complementary information and different applications. Generic profile based QoL can not be used in economic evaluation. Therefore, it is advised to use several HRQoL measures (23). For example, both generic and specific HRQoL measures are more useful than utilities in the identification of the dimensions for which there are improvements and decrements. In addition, specific HRQoL measures may be more responsive to small changes (13).

By measuring the responsiveness of a HRQoL instrument, some judgment is possible about the ability to detect changes that are meaningful or clinically important. When a HRQoL instrument is more responsive, it has the advantage of requiring smaller sample sizes to demonstrate clinically important effects (24). 
The ultimate goal in the rehabilitation of stroke survivors is to regain independence and improve quality of life. To reach this goal, regaining the ability to walk again is very important, as physical inactivity is highly correlated with morbidity (21). One of the motor impairments which are commonly seen in stroke survivors is a drop foot. A drop foot hinders them to walk normally, resulting in an abnormal, inefficient, and unsafe gait. Besides the conventional treatments like an ankle-foot orthosis or orthopedic footwear, peroneal nerve stimulation is one of the treatment devices that can be used to correct a drop foot. The present study focuses on the effect of a mobility intervention for drop foot correction, that is, an implantable two-channel peroneal nerve stimulator, on HRQoL. The first goal was to examine if the implantable two-channel peroneal nerve stimulator improves HRQoL. The second goal was to investigate both the internal and external responsiveness of the preference-based utility measures included in the present study. The third and last goal was to examine if a relation was present between the different preference-based utility outcomes, measured both in a direct (TTO) and indirect way (EQ-5D and SF-36). Our hypothesis was that the use of the implantable two-channel peroneal nerve stimulator will improve the HRQoL of stroke subjects with a drop foot. 


\section{Methods}

\section{Study design}

The study was conducted as a randomized controlled trial and the CONSORT statement was used to report the trial (25). In addition to the primary clinical end points, several measures were taken in the present study to include all aspects of HRQoL. HRQoL was assessed in two different ways: (i) by taking descriptive measures, that is, the SF-36 (generic measure) and the DIP (disease-specific measure); and (ii) by obtaining preference-based utilities both measured with the TTO (direct way) and by calculating them from the EQ-5D and SF-36. All subjects were assessed three times during the study. The baseline measurement took place about 4 weeks before the randomization procedure and the follow-up measurements were performed at 12 and at 26 weeks after the surgical procedure in the intervention group. Subjects assigned to the control group were assessed in the same weeks as subjects assigned to the intervention group. All assessments were performed by the same examiner (A.K.). Three weeks after the implantation procedure, subjects received the transmitter and instructions were given on the proper use of the peroneal nerve stimulator. Four and 8 weeks after the implantation procedure, the intervention group was also invited to assess the stimulation levels of the two output channels. If problems were experienced by the subjects, they were also solved. The control group was also seen in the same weeks, to keep the number of visits the same for both study groups so that the same amount of attention was paid to both groups. Blinding of both the study personnel and participants was not possible due to the surgical procedure. All data were analyzed according to an intent-to-treat principle.

\section{Subjects}

Most subjects were recruited in response to an article in a local Dutch newspaper about the first results with the implantable stimulator in stroke survivors. Some subjects were recruited through consultant and general practitioner referrals and, in some cases, on the advice of the physiotherapist treating the subject. Both recruitment strategies resulted in a selected sample of well-motivated stroke survivors. Because of the invasive nature of the treatment, selection of the most appropriate subjects is very important. Therefore, subjects that were most likely to experience treatment success were selected. Table 6.1 shows the criteria for inclusion and exclusion in the trial. 
Table 6.1 Selection criteria

\begin{tabular}{ll}
\hline Inclusion criteria & Exclusion criteria \\
\hline $\begin{array}{l}\text { Drop foot identified by an inability to achieve a } \\
\text { normal heel strike during walking }\end{array}$ & Age $<18$ year \\
$\begin{array}{l}\text { First hemiplegia of at least } 6 \text { months duration as a } \\
\text { result of a CVA with a stable neurology } \\
\text { Subject is an outdoor walker }\end{array}$ & $\begin{array}{l}\text { Passive dorsiflexion of the ankle }<5 \text { o with knee in } \\
\text { extension } \\
\text { Medical conditions other than CVA, i.e. neurological, } \\
\text { rheumatic, cardio-vascular or systemic disorders } \\
\text { (including Diabetes Mellitus) limiting the function of } \\
\text { walking }\end{array}$ \\
& $\begin{array}{l}\text { Injury to deep and superficial peroneal nerve and } \\
\text { sciatic nerve } \\
\text { Able to give an informed consent }\end{array}$ \\
& $\begin{array}{l}\text { Any medical condition that would exclude the use of } \\
\text { a surgical procedure or anesthetic } \\
\text { Not able to don and doff the equipment } \\
\text { Pregnancy }\end{array}$ \\
\hline
\end{tabular}

Subjects that fulfilled the selection criteria were admitted to the trial. One week after the baseline assessment, subjects were randomly allocated to either the intervention group or control group. Random allocation was performed in blocks of two subjects, to ensure a close balance of the numbers in each group. The randomization procedure was performed by an independent individual. Each time two suitable subjects were included in the study, their names were given to the person who was responsible for the randomization procedure. This person informed the examiner, after the baseline measurement was performed, which subject was allocated to the intervention group and which subject was allocated to the control group. All subjects were allowed to continue their usual (physio)therapy sessions during the trial, which was recorded in a subject diary.

The experiment protocol was approved by the local Medical ethics committee and the subjects gave their informed consent before participating in the experiments.

\section{Stimulation system}

The implantable two-channel peroneal nerve stimulator was fabricated by Finetech Medical Limited ${ }^{1}$ and consists of an external transmitter with a built-in antenna, a foot switch, and an implantable part consisting of the stimulator, the two leads, and the bipolar intraneural electrodes $(26,27)$.

The transmitter weighs approximately $0.1 \mathrm{~kg}$ and is attached with a strap on the lateral side of the lower leg, over the site of the implant, just below the knee. A foot switch 
placed under the heel of the subject's foot inside the shoe determines the on-and-off switching of the stimulation. Over night, the battery of the transmitter is charged.

The implantable part is a passive device, receiving information carried by the radio frequency signals and converting them into the stimulation pulses of the desired amplitude and frequency. One electrode is placed below the epineurium of the superficial peroneal nerve (eversion) and one below the epineurium of the deep peroneal nerve (dorsiflexion). For a more detailed description of the implantable stimulator, see Kottink et al. (28).

\section{Measurements}

\section{Descriptive HRQoL measures}

Both the SF-36 (generic measure) and the DIP (specific measure) were taken in the present study to measure HRQoL.

SF-36. The SF-36 is a reliable and valid instrument $(17,29)$ and is designed to be applicable to a wide range of types and severities of conditions. It includes multi-item scales to measure the following eight dimensions: physical functioning, role limitations due to physical health problems, bodily pain, social functioning, general mental health covering psychological distress and well-being, role limitations due to emotional problems, vitality, energy or fatigue, and general health perceptions. Besides calculating the subscale scores, also the physical and mental component summary scores were calculated, referred to as PCS-36 and MCS-36, respectively. The SF-36 can be selfadministered or used in personal or telephone interviews.

DIP. The DIP is a self-administered questionnaire, that was developed mainly on the basis of the Disability and Handicap sections of the World Health Organization's International classification of Impairments, Disabilities, and Handicaps (30). The questionnaire contains three symptom questions and 36 disability questions on five different domains: mobility, self-care, social activities, communication, and psychological status. Each disability question is followed by a question in which the subject is asked to rate the importance or impact of that particular disability, resulting together in a weighted score. Scores for each domain were computed according to the method described by Lankhorst et al. (30). 


\section{Preference-based utility measures}

Besides descriptive HRQoL instruments, also preference-based utilities were obtained in the present study, both measured by the TTO and calculated from the EQ-5D and SF36.

TTO. The TTO is a widely used method to measure health-state preferences (22). Utility scores for both present hemiplegic health state (ТТОн) and present hemiplegic health state with normal walking function (ТТОннw) were determined using the TTO technique. Subjects were asked to imagine that they have 1 year to live and that they have to make a choice between living this year in the present hemiplegic health state (with all health problems they experience) and taking an imaginary drug that will cure a specific condition. However, the disadvantage of this imaginary drug is that they will live for less than 1 year. It was systematically assessed how many months the subjects were willing to trade for a cure of all their hemiplegic related health problems (ТТОн) or a cure of their walking dysfunction (ТТОннw). For both parts, scores could range from 0 to 1.0 .

$E Q-5 D$. The EQ-5D generic health index is a standardized instrument that is applicable to a wide range of health conditions and treatments (16). The first part records selfreported problems on the domains mobility, self-care, usual activities, pain/discomfort, and anxiety/depression. Each domain is divided into three levels of severity corresponding to no problem, some problem, and extreme problem. The second part comprises a visual analog scale (VAS), described as a "feeling thermometer" rated from 0 (worst imaginable health state) to 100 (best imaginable health state). The EQ-5D is designed for self-completion by respondents. In the present study, the EQ-5D was used to calculate a preference-based summary index based on TTO techniques in which the value 0 represents death and 1 represents perfect health.

SF-36. Besides providing a simple descriptive profile, the SF-36 was used to calculate a preference-based summary index based on the standard gamble approach in which the value 0 represents death and 1 represents perfect health (31).

One week before the baseline, week 12 and week 26 assessment, the SF-36, the DIP, and the EQ-5D were sent to the house of the subjects. Because none of the included 
stroke subjects had significant cognitive deficits, they were able to self-administer the questionnaires at home, without the help of the examiner. This was done for two reasons: (i) to give them enough time to fill in the questionnaires and (ii) to let them feel comfortable by being in their own home environment. At the assessments, the examiner checked if all questionnaires were filled in by the subjects. If this was not the case, subjects were asked to fill in their answer after the question was explained to them by the examiner. Also, the TTO was scored at both assessments, after instructions were given to the subject by the examiner.

\section{Statistical analysis}

An analysis of covariance was used to test if differences in all HRQoL measures were present between the intervention and control group at week $26(32,33)$. To correct for possible differences at baseline between the intervention and control group, the baseline scores were taken as covariate. Group was taken as the only fixed factor in the analysis. Normality plots of the residuals were made to examine if the data were distributed according to a normal distribution.

The internal responsiveness of the included preference-based utility measures, which characterizes the ability of these measures to detect clinically important HRQoL changes, was compared by calculating Cohen's effect sizes for both the intervention and control group. Effect sizes were calculated as the ratio between mean change scores and the standard deviation (sd) of baseline scores (34). An independent t-test was used to make a comparison between the effect size found for both the intervention and control group.

The external responsiveness of the included preference-based utility measures, which reflects the extent to which longitudinal changes in these HRQoL measures over time relate to corresponding changes in a reference measure of health status, was assessed by using a linear regression model (35). In the present study, the VAS of the EQ-5D, which provides a single index value for health status, was selected as a reference measure for detecting change.

Pearson's correlation coefficients $(\mathrm{r})$ were calculated to examine if the different utility outcomes, obtained from the TTO, EQ-5D, and SF-36, were related to each other in both the intervention and control group at baseline, week 12 and week 26. For the interpretation of the size of correlation coefficients, the guideline of Cohen was used 
(36) (0.1-0.29: small correlation; 0.3-0.49: medium correlation, and 0.5-1.0: large correlation).

All statistical analyses were performed with SPSS version 11.5 for Windows. The significance level a was set at 0.05 for all tests. 


\section{Results}

\section{Participants}

Figure 6.1 shows the flow of participants throughout the study. Twenty-nine subjects with drop foot due to cerebrovascular accident participated in the present study. Table 6.2 shows the characteristics of the participants. Subject characteristics were not very different between both groups, with the exception of mean time after stroke, which was longer for the intervention group.

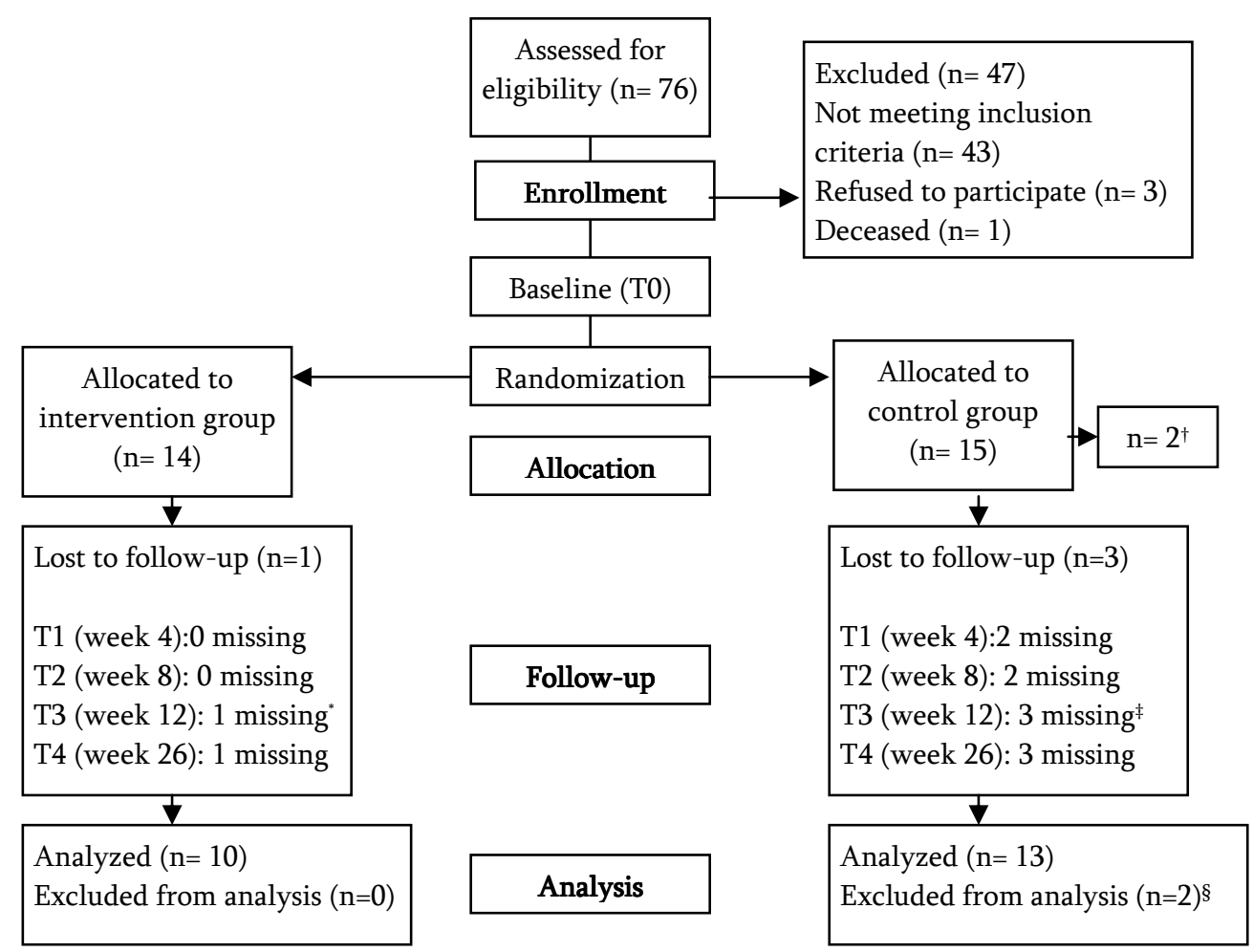

Figure 6.1 Flowchart of patients through the study

*Defective implant

${ }^{\dagger}$ Resultant of randomization procedure

₹ Psychologic issues not related to the study

$\S$ Excluded from analysis as a result of drop-out after randomization 
In the intervention group, seven subjects used a plastic ankle-foot orthosis, three subjects wore orthopedic shoes, and four subjects did not use a walking aid to correct their drop foot just prior to the start of the trial. In the control group, all subjects wore a plastic ankle-foot orthosis. Four subjects dropped out of the study, one woman in the intervention and three men in the control group. The implant of the subject in the intervention group who dropped out failed after functioning properly for about 10 weeks. An investigation of the explanted system showed that the failure was caused by a technical defect in the epineural electrode responsible for the dorsiflexion movement. Two subjects in the control group withdrew after the randomization procedure because they were disappointed about the outcome, and the other subject dropped out in week 11, because of psychological issues not related to the study. The remaining subjects in the intervention group did not report any technical failure of the stimulation system and continued to use the stimulator during the entire follow-up period. Besides the subjects who dropped out of the study, in both the intervention and control group, two subjects did not fill in the TTO during the assessments. Two subjects, one of each group, were angry about the unrealistic scenario that was stated; the other two subjects, also one of each group, were not able to make the judgment.

Table 6.2 Subject characteristics

\begin{tabular}{lcc}
\hline & Intervention group & Control group \\
\hline Number of subjects (drop outs) & $14(1)$ & $15(3)$ \\
Men & 10 & 10 \\
Women & 4 & 5 \\
Mean age (sd) & 55.2 years $(11.36)$ & 52.87 years $(9.87)$ \\
Mean time after stroke (sd) & 9.07 years $(9.29)$ & 5.67 years $(4.64)$ \\
Left side affected & 7 & 9 \\
Right side affected & 7 & 6 \\
\hline
\end{tabular}

\section{Descriptive HRQoL measures}

$S F-36$

Table 6.3 shows the domain scores (mean values) of the SF-36 for both the intervention and control group during baseline and week 26. On the physical functioning domain, the intervention group changed $11.9(\mathrm{sd}=14.7)$ in score, while the control group changed -5.4 (sd=16.6) in score, which resulted in a significant difference between both groups at week $26(\mathrm{p}<.01)$. Also, on the domain general health and the PCS-36, a significant difference between both groups was found at week $26(\mathrm{p}<.05$ and $\mathrm{p}<.01$, respectively). On the general health domain, the intervention group changed 9.1 
$(s d=12.8)$ and the control group changed $-6.3(\mathrm{sd}=16.2)$ in score, while on the PCS-36, the scores changed $4.1(\mathrm{sd}=6.9)$ and $-2.6(\mathrm{sd}=7.9)$, respectively. On the domains physical role functioning, bodily pain, social functioning, mental health, emotional role functioning, vitality, and on the MCS-36, no significant differences between both groups were found.

Table 6.3 Domain scores of SF-36 for intervention and control group (mean values (sd))

PCS-36= physical component summary score; MCS-36= mental component summary score

\begin{tabular}{lccccc}
\hline Functional domains & \multicolumn{2}{c}{ Intervention } & \multicolumn{2}{c}{ Control } & p \\
& Baseline & Week 26 & Baseline & Week 26 & \\
\hline Physical functioning & $45(18.3)$ & $57.3(20.2)$ & $45.7(13.1)$ & $40.8(14.3)$ & .008 \\
Physical role functioning & $37(43.1)$ & $44.2(42.3)$ & $53.3(37.6)$ & $33.3(32.6)$ & .442 \\
Bodily pain & $76.1(23.8)$ & $78.2(24.7)$ & $69.1(20.2)$ & $69.9(19.8)$ & .509 \\
Social functioning & $70.5(24.8)$ & $75(24.5)$ & $80.8(18.8)$ & $67.7(24.7)$ & .190 \\
Mental health & $68.6(22.5)$ & $72.9(19.3)$ & $75.2(12.6)$ & $73.3(15.6)$ & .809 \\
Emotional role functioning & $52.4(50.2)$ & $61.5(40.5)$ & $82.2(33)$ & $86.1(33.2)$ & .592 \\
Vitality & $60(21.12)$ & $60.8(19.7)$ & $61.7(12.3)$ & $53.6(20.6)$ & .389 \\
General health & $60(20.6)$ & $70(24.8)$ & $69.6(15.6)$ & $66.7(17)$ & .041 \\
PCS-36 & $38.6(6.8)$ & $42.5(7.3)$ & $38.3(8.8)$ & $34.4(5.9)$ & .004 \\
MCS-36 & $46.8(14.1)$ & $48.4(12.1)$ & $53.7(7.9)$ & $52.7(11.2)$ & .85 \\
\hline
\end{tabular}

\section{$D I P$}

Figure 6.2 shows the domain scores of the DIP for both the intervention and control group during baseline and week 26. On all domains, except for the symptoms domain, the scores in the intervention group changed positively, while the scores for the control group changed negatively during the study. For the domains mobility, self-care, and psychological status, a significant difference between both groups was found at week 26 (respectively $\mathrm{p}=.006, \mathrm{p}=.00$, and $\mathrm{p}=.025$ ). No differences between both groups were found on the domains symptoms, social activities and communication. 


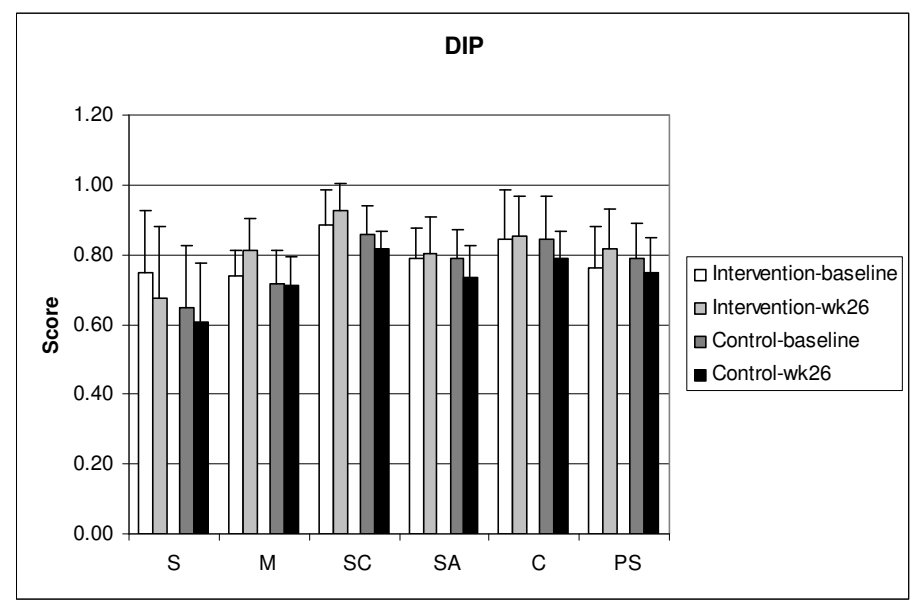

Figure 6.2 Domain scores of DIP for intervention and control group (mean values (sd)). S, symptoms; M, mobility; SC, self-care; SA, social activities; C, communication, PS, psychological status

\section{Preference-based utility measures}

TTO

Utility scores of $0.84(\mathrm{sd}=.23)$ and $0.83(\mathrm{sd}=.21)$ were found for, respectively, the intervention and control group for ТТОн at baseline. At week 26, mean scores of 0.88 $(\mathrm{sd}=.10)$ and $0.89(\mathrm{sd}=.13)$ were found for the intervention and control group. No significant difference was found between both groups on ТТОн ( $\mathrm{p}=.57)$. For ТТОннш, the utility score of the intervention group changed from 0.75 ( $\mathrm{sd}=.24)$ to 0.94 ( $\mathrm{sd}=.08$ ), while the score of the control group changed from $0.86(\mathrm{sd}=.20)$ to $0.92(\mathrm{sd}=.14)$. Again, no significant difference was found between both groups during the trial $(\mathrm{p}=.70)$.

\section{$E Q-5 D$}

At baseline, mean preference-based summary indexes of $0.70(\mathrm{sd}=.21)$ and $0.67(\mathrm{sd}=.12)$ were found for, respectively, the intervention and control group. At week 26, the index shown by the intervention group changed to a mean value of $0.76(\mathrm{sd}=.11)$. The control group showed an index of $0.66(\mathrm{sd}=.08)$ at the final assessment. Overall, a significant difference between both groups was found at week 26 ( $p=.017)$.

\section{$S F-36$}

The mean preference-based summary index for the intervention and control group were, respectively, $0.67(\mathrm{sd}=.13)$ and $0.69(\mathrm{sd}=.11)$ at baseline. At week 26, the index 
found for the intervention group changed to a mean value of $0.71(\mathrm{sd}=.14)$, while the index found for the control group changed to a mean value of $0.64(\mathrm{sd}=.05)$. However, no significant difference between both groups was found $(\mathrm{p}=.15)$.

\section{Responsiveness}

\section{Internal responsiveness}

Table 6.4 shows the internal responsiveness statistics of the preference-based utility measures. As can be seen from the table, for the intervention group, higher effect sizes were found for all preference-based utility measures than for the control group, with exception of TTOн. Table 6.4 shows that both the EQ-5D and the SF-36 were the most responsive utility measures.

\section{External responsiveness}

With the external responsiveness, statistics was examined if changes in the included preference-based utility measures over time were related to corresponding changes in the VAS of the EQ-5D. $R 2$ for TTOн, TTOнnw, EQ-5D, and SF-36 were, respectively, $0.15(\mathrm{p}=.07), 0.001(\mathrm{p}=.91), 0.16(\mathrm{p}=.09)$, and $0.09(\mathrm{p}=.19)$.

Table 6.4 Internal responsiveness statistics of preference-based utility measures

\begin{tabular}{lccc}
\hline & Intervention & Control & T-test \\
\hline TTOн & 0.23 & 0.45 & $\mathrm{p}=.58$ \\
TTOHNw & 0.62 & 0.50 & $\mathrm{p}=.73$ \\
EQ-5D & 0.44 & -0.04 & $\mathrm{p}=.27$ \\
SF-36 & 0.29 & -0.22 & $\mathrm{p}=.26$ \\
\hline
\end{tabular}

\section{Correlation between preference-based utility measures}

Large Pearson's correlation coefficients were found between the utilities calculated from the EQ-5D and SF-36 at baseline and at week 12, respectively 0.66 and 0.52 . A medium correlation coefficient of 0.39 was found at week 26 between the utilities calculated from the EQ-5D and SF-36. The remaining correlation coefficients were small. 


\section{Discussion}

This study focused primarily on the evaluation of a mobility intervention for drop foot correction, that is, an implantable two-channel peroneal nerve stimulator, on HRQoL. Two descriptive instruments, a generic and a specific, were included in the present study to examine if they resulted in a comparable HRQoL outcome.

The results of the present study show a significant effect of the implantable peroneal stimulator on the domains physical functioning, general health, and the PCS-36 of the SF-36. This finding was in agreement with our expectation, as an earlier study showed that the use of the implantable device results in an improved walking function (28).The SF-36, a generic descriptive instrument, seems, therefore, to be a good tool to assess this specific improvement on physical health. The SF-36 results of the present study were in agreement with Fernandes et al. (10), who reported that a functional electrical orthosis applied over the paretic leg of stroke subjects is able to improve QoL, mainly physical functioning. To their opinion, physical functioning is, besides using it for the evaluation of an intervention, extremely important information for physiotherapy and for the preparation of a rehabilitation program, as it shows how the motor disability and sensitiveness caused by the lesion interferes in daily activities. In contrast to the findings found in the present study and the findings of Fernandes et al. (10), Rydwik et al. (11), who evaluated the effect of an ankle-exercise intervention program with a portable device, showed no significant effect on HRQOL measured with the SF-36.An important limitation of the study of Rydwik et al. (11) was that the sample size was not sufficient to detect significant changes between the control and intervention group. They concluded that the study must be repeated using a more targeted group of stroke subjects before any conclusions can be drawn. In addition, it is known from the literature that the SF-36 is known with high ceiling effects on some domains in the stroke population, which make it also difficult to detect significant changes (37).

On the mobility domain of the disease-specific DIP questionnaire, which was of primary interest for the present study, also a significant effect of the implantable device was found. Also, a significant positive effect was found on the domains social activities and psychological status in the intervention group. The DIP was originally designed for Multiple Sclerosis subjects and, as far as we know, no literature is known about the use of this questionnaire in chronic stroke subjects. 
From the results, it becomes clear that both descriptive measures included in the present study, a general and a disease specific, result in a similar improvement of HRQoL, that is, a significant effect on the domains related to physical health.

Besides descriptive instruments, also some preference-based utility measures were used to assess changes in HRQoL after using a drop foot implant. When looking at the TTO results, it became clear that ТТОн and ТТОннw did not show a significant effect of the implantable device. One of the factors that could have influenced the judgment of our participants was the emotional response that was triggered during the assessment of the TTO. This was also described by Lenert and Kaplan (14) as a possible factor that can influence measurements of utilities. The unrealistic scenario that was stated made them a bit angry and, as a consequence, two participants refused to fill in the TTO at all. Two other participants found the TTO technique too difficult and therefore were not able to make the judgment. Therefore, the indirect way to calculate a utility index may have been more successful in the present study, as none of the participants reported any problems with filling in the EQ-5D and SF-36. A remarkable finding of our TTO results was that at baseline, eight subjects were willing to trade more months for a cure of their walking dysfunction (ТТОнмw) than for a cure of all their hemiplegic related health problems (ТТОн), which, of course, also includes the cure of their walking dysfunction. At week 26, this remarkable finding was still valid for two subjects. A reasonable explanation for this can be that the description of a more explicit condition, that is, the cure of the walking dysfunction versus the cure of all hemiplegic-related health problems, has more impact on a subject because he knows exactly what he can expect from taking the imaginary drug. When all hemiplegic-related health problems that would have been cured by the imaginary drug were explicitly described at TTOH, subjects would probably have traded more months than was the case nowadays.

Regarding the utility indexes calculated from the EQ-5D and SF-36, the same trend was shown by both the intervention and control group. At the end of the study, the utility index was higher for the intervention group, while the utility index was lower for the control group when compared with baseline. However, only a positive significant effect of the implantable device was found on the utility index calculated from the EQ5D.Wee et al. (38) reported that clinically important differences can be found in utility scores when various preference-based instruments are used. These differences in outcome can be explained by the methodology that is used to estimate preference scores. For the EQ-5D, the TTO approach is used to calculate utilities (39), while for the 
SF-36, the standard gamble approach is used $(31,40)$. As a consequence, the utilities calculated from the SF-36 are, in general, higher than those calculated from the EQ-5D $(38,41,42)$. In this study, the utilities calculated from both questionnaires were relatively the same.

Because different approaches were used in the present study to obtain utility scores, that is, a TTO method, a questionnaire with a preference-based summary index based on the TTO method (EQ-5D) and a questionnaire with a preference-based summary index based on the standard gamble method (SF-36), it is interesting to examine which preference-based utility measure is the most responsive instrument. By knowing the responsiveness, some judgment is possible about the ability to detect changes that are meaningful or clinically important. When a preference-based utility measure is more responsive, it has the advantage of requiring smaller sample sizes to demonstrate clinically important effects (24). From the results, it became clear that both EQ-5D and SF-36 were in an equal way able to detect changes that are meaningful or clinically important. This finding is in agreement with results reported by Pickard et al., who also studied the responsiveness of some preference-based generic measures of HRQoL in stroke subjects (24). They also found that the indices of responsiveness for the utilities calculated from the EQ-5D and the SF-36 were of similar magnitude in most instances. In their study, also the responsiveness of the VAS scale of the EQ-5D was calculated. In comparison to the EQ-5D and the SF-36, this outcome measure is less responsive and requires larger sample sizes to detect important differences. This can be an explanation for our external responsiveness statistics, where no correspondence was found between the changes found in the included preference-based utility measures and the changes on the VAS of the EQ-5D.

The last goal of our study was to examine if a relation was present between different preference-based utility outcomes, measured both in a direct (TTO) and indirect way (EQ-5D and SF-36). With respect to the correlation between preference-based utility measures, significant relations were found between the utility index calculated from the EQ-5D and SF-36. In the past, Dorman et al. (43) made a comparison between responses to the EQ-5D and SF-36 by a group of subjects after stroke to examine the absence or presence of a relationship between both instruments. A large correlation was found for the domains of both instruments which assessed physical functioning, social functioning, bodily pain, and overall HRQoL. Only the mental health domain of the SF36 correlated poorly with the psychological functioning domain of the EQ-5D.With 
these results in mind, it is not surprising that a significant correlation was found between both instruments in our study.

\section{Study limitations}

The sample size of the present study was relatively small, which further limits generalizability to the broader population with stroke and placing the study at risk for false-negative results (type II error). Regarding the subject characteristics, it was a coincidence that, after randomization, the mean time after stroke in the intervention group was about $60 \%$ longer than in the control group. However, we do not expect that this had any influence on our results, since all subjects are in the chronic phase of stroke.

This study demonstrates that the implantable two-channel peroneal nerve stimulator seems to improve HRQoL, which makes it a good treatment option for a selected subpopulation of drop foot patients who had suffered an upper motor neuron lesion. This finding was also in correspondence with an earlier study (28). For the development of an evidence-based stroke rehabilitation regarding the correction of drop foot, an economic evaluation is advised in the future. By means of a cost-utility analysis, where healthy effects are expressed in QALYs, a comparison can be made between the different drop foot treatments that are available in clinical practice. Only in this way will a good understanding be obtained about which treatment option is the most efficient one.

\section{Conclusion}

The implantable two-channel peroneal nerve stimulator seems to be efficient to improve HRQoL, mainly in the domains related to physical functioning. The preference-based HRQoL summary scores derived from the EQ-5D and the SF-36 were generally more responsive than the scores obtained by the TTO method to detect important differences. The utility indexes calculated from the EQ-5D and SF-36 were also related to each other. 


\section{References}

1. Kwa VI, Limburg M, de Haan RJ. The role of cognitive impairment in the quality of life after ischaemic stroke. J Neurol 1996;243:599-604.

2. Gotay CC, Korn EL, McCabe MS, Moore TD, Cheson BD. Quality-of-life assessment in cancer treatment protocols: research issues in protocol development. J Natl Cancer Inst 1992;84:575-9.

3. Niemi ML, Laaksonen R, Kotila M, Waltimo O. Quality of life 4 years after stroke. Stroke 1988;19:1101-7.

4. Viitanen M, Fugl-Meyer KS, Bernspang B, Fugl-Meyer AR. Life satisfaction in long-term survivors after stroke. Scand J Rehabil Med 1988;20:17-24.

5. Indredavik B, Bakke F, Slordahl SA, Rokseth R, Haheim LL. Stroke unit treatment improves long-term quality of life: a randomized controlled trial. Stroke 1998;29:895-9.

6. King RB. Quality of life after stroke. Stroke 1996;27:1467-72.

7. Haacke C, Althaus A, Spottke A, Siebert U, Back T, Dodel R. Long-term outcome after stroke: evaluating health-related quality of life using utility measurements. Stroke 2006;37:193-8.

8. Ahlsio B, Britton M, Murray V, Theorell T. Disablement and quality of life after stroke. Stroke 1984;15:886-90.

9. Anderson C, Laubscher S, Burns R. Validation of the Short Form 36 (SF-36) health survey questionnaire among stroke patients. Stroke 1996;27:1812-6.

10. Fernandes MR, Carvalho LB, Prado GF. A functional electric orthesis on the paretic leg improves quality of life of stroke patients. Arq Neuropsiquiatr 2006;64:20-3.

11. Rydwik E, Eliasson S, Akner G. The effect of exercise of the affected foot in stroke patients—a randomized controlled pilot trial. Clin Rehabil 2006;20:645-55.

12. Langhammer B, Stanghelle JK, Lindmark B. Exercise and health-related quality of life during the first year following acute stroke. A randomized controlled trial. Brain Inj 2008;22:135-45.

13. Feeny D. A utility approach to the assessment of health-related quality of life. Med Care 2000;38 (9 Suppl):II151-4.

14. Lenert L, Kaplan RM. Validity and interpretation of preference-based measures of healthrelated quality of life. Med Care 2000;38 (9 Suppl):II138-50.

15. Tsevat J. What do utilities measure? Med Care 2000;38 (9 Suppl):II160-4.

16. Hunt SM, McKenna SP, McEwen J, Williams J, Papp E. The Nottingham Health Profile: subjective health status and medical consultations. Soc Sci Med [A] 1981;15:221-9.

17. Ware JE Jr, Sherbourne CD. The MOS 36-item short-form health survey (SF-36). I. Conceptual framework and item selection. Med Care 1992;30:473-83. 
18. Lankhorst GJ, Jelles F, Smits RC, et al. Quality of life in multiple sclerosis: the disability and impact profile (DIP). J Neurol 1996;243:469-74.

19. Bult JR, Hunink MG, Tsevat J,Weinstein MC. Heterogeneity in the relationship between the time tradeoff and short form-36 for HIV-infected and primary care patients. Med Care 1998;36:523-32.

20. Gold MR, Siegel JE, Russell LB, Weinstein RW. Cost-Effectiveness in Health and Medicine. New York: Oxford University Press, 1996.

21. Mortimer D, Segal L. Comparing the incomparable? A systematic review of competing techniques for converting descriptive measures of health status into QALY-weights. Med Decis Making 2008;28:66-89.

22. Daly JJ, Ruff RL. Construction of efficacious gait and upper limb functional interventions based on brain plasticity evidence and model-based measures for stroke patients. Scientific World Journal 2007;7:2031-45.

23. Feeny D. A utility approach to the assessment of health-related quality of life. Med Care 2000;38 (9 Suppl):II151-4.

24. Pickard AS, Johnson JA, Feeny DH. Responsiveness of generic health-related quality of life measures in stroke. Qual Life Res 2005;14:207-19.

25. Altman DG, Schulz KF, Moher D, et al. The revised CONSORT statement for reporting randomized trials: explanation and elaboration. Ann Intern Med 2001;134:663-94.

26. Holsheimer J, Bultstra G, Verloop AJ, van der Aa HE, Hermens HJ. Implantable dual channel peroneal nerve stimulator. Proceedings Ljubljana FES Conference, Ljubljana, Slovenia, 1993;42-4.

27. van der Aa HE, Bultstra G,Verloop AJ, et al. Application of a dual channel peroneal nerve stimulator in a patient with a "central” drop foot. Acta Neurochir Suppl 2002;79:105-7.

28. Kottink AI, Hermens HJ, Nene AV, et al. A randomized controlled trial of an implantable 2-channel peroneal nerve stimulator on walking speed and activity in post-stroke hemiplegia. Arch Phys Med Rehabil 2007;88:971-8.

29. McHorney CA, Ware JE Jr, Raczek AE. The MOS 36-Item Short-Form Health Survey (SF36): II. Psychometric and clinical tests of validity in measuring physical and mental health constructs. Med Care 1993;31:247-63.

30. Lankhorst GJ, Jelles F, Smits RC, et al. Quality of life in multiple sclerosis: the disability and impact profile (DIP). J Neurol 1996;243:469-74.

31. Brazier J, Roberts J, Deverill M. The estimation of a preference-based measure of health from the SF-36. J Health Econ 2002;21:271-92.

32. Senn S. Statistical Issues in Drug Development. Glasgow. JohnWiley \& Sons Ltd, 1997.

33. Van Breukelen GJ. ANCOVA versus change from baseline: more power in randomized studies, more bias in nonrandomized studies. J Clin Epidemiol 2006;59:920-5. 
34. Kazis LE, Anderson JJ, Meenan RF. Effect sizes for interpreting changes in health status. Med Care 1989;27 (3 Suppl):S178-89.

35. Husted JA, Cook RJ, Farewell VT, Gladman DD. Methods for assessing responsiveness: a critical review and recommendations. J Clin Epidemiol 2000;53:459-68.

36. Cohen J. Statistical Power Analysis for the Behavioral Sciences. Hillsdale, NJ: Lawrence Erlbaum Associates, 1988.

37. Buck D, Jacoby A, Massey A, Ford G. Evaluation of measures used to assess quality of life after stroke. Stroke 2000;31:2004-10.

38. Wee HL, Machin D, Loke WC, et al. Assessing differences in utility scores: a comparison of four widely used preference-based instruments. Value Health 2007;10:256-65.

39. Dolan P. Modeling valuations for EuroQol health states. Med Care 1997;35:1095-108.

40. Nichol MB, Sengupta N, Globe DR. Evaluating quality-adjusted life years: estimation of the health utility index (HUI2) from the SF-36. Med Decis Making 2001;21:105-12.

41. Kopec JA, Willison KD. A comparative review of four preference-weighted measures of health-related quality of life. J Clin Epidemiol 2003;56:317-25.

42. Hawthorne G, Richardson J, Day NA. A comparison of the Assessment of Quality of Life (AQoL) with four other generic utility instruments. Ann Med 2001;33:358-70.

43. Dorman PJ, Dennis M, Sandercock P. How do scores on the EuroQol relate to scores on the SF-36 after stroke? Stroke 1999;30:2146-51.

\section{Suppliers}

${ }^{1}$ Finetech Medical Limited, 13 Tewin Court, Welwyn Garden City, Hertfordshire, AL7 $1 \mathrm{AU}$, England. 

Chapter 7

General Discussion 



\section{General Discussion}

A problem which is often seen after stroke is a drop foot, which is defined as a significant weakness or absence of ankle and toe dorsiflexors. Besides conventional splinting treatments like an ankle foot orthosis (AFO) or orthopaedic shoes, also a neural prosthesis can be used to improve this lost body function. A neural prosthesis is an artificial system bypassing the neural system to improve lost body functions by providing functional movement patterns generated by electrical stimulation (ES) (1). An example of a neuroprosthesis is a peroneal nerve stimulator (PNS), which stimulates the dorsal flexors of the foot during the swing phase of gait. In stroke rehabilitation, peroneal nerve stimulation (PNS) can have two different functions. First it can be used to stimulate muscles to prevent a drop foot during walking (orthotic effect) (2-6). Second, there is also some evidence that it can be used to modulate the neuromuscular system in the long term, resulting in therapeutic effects (6-9). Therapeutic ES is used to minimize specific impaired body functions, such as limited range of motion, motor weakness and spasticity (10).

At the start of the present thesis, no clear evidence was available regarding improvement of hemiplegic gait with regard to both the AFO and PNS, due to lack of rigorously controlled trials (11). The goal of this thesis was to study both the orthotic and therapeutic effect of an implantable two-channel peroneal nerve stimulator, in comparison with the conventional treatment, in chronic stroke survivors with a drop foot in a randomized controlled trial (RCT).

To obtain more insight in the orthotic effect of PNS on the improvement of walking in stroke subjects with a drop foot, a systematic review was performed, described in chapter 2 of this thesis. From the review it became clear that the literature justifying the use of stimulation to correct a drop foot is mainly based on case studies, uncontrolled trials and retrospective reviews. Only one RCT was performed (9). In this RCT a comparison was made between walking with and without stimulation, e.g. the orthotic effect in the same patients that used both methods.

More interestingly, is to examine the effect of PNS by comparing patients who only use PNS as a first-line treatment with patients only receiving conventional orthotic treatment. To progress towards evidence-based application of PNS versus conventional splinting to improve lower extremity function after stroke, this $\mathrm{PhD}$ thesis focussed on 
the results found in a RCT (chapters 3-6). An implantable two-channel peroneal nerve stimulator (STIMUSTEPTM), which stimulates both the deep and superficial branches of the common peroneal nerve, was used to generate muscular contractions of the dorsalflexors and evertors with the purpose to correct a drop foot. In this chapter the main findings of the RCT will be discussed. Also recommendations for daily clinical practice and future studies will be given.

\section{Orthotic effect of PNS}

In the present RCT, different outcome measures like walking speed, hemiplegic gait pattern, physical activity and health-related quality of life (HRQOL), were measured to study the 'orthotic effect' of PNS. The orthotic effect can be defined as the effect that is measured while the stimulator is used.

\section{Walking speed}

From the review described in chapter 2 it became clear that most studies, that examined the effect of PNS, focused on walking speed as primary outcome measure, measured on a 10-meter walkway. Walking speed is fairly easy to measure and it is also a valid, reliable and responsive outcome measure (12;13). Since functional outcome measures are less consistently used in clinical reports and have shown more heterogeneity, walking speed is recommended as a primary endpoint (14;15). After pooling of the included studies, a pooled effect size of $0.13 \mathrm{~m} / \mathrm{s}(0.07-0.2 \mathrm{~m} / \mathrm{s})$ or $38 \%(22.18-53.8 \%)$ was found, which means that PNS results in a positive effect on walking speed.

In chapter 3 the orthotic effect of implantable PNS on walking speed was chosen as primary outcome measure. Besides measuring walking speed on a 10- meter walkway, walking speed was also calculated from a 6-minute walk test (6MWT) (16). In comparison with the 10-meter walkway, the longer duration of the 6MWT might reflect a more natural cadence and velocity. Also a fatigue effect might be induced as a result of the longer duration. To our opinion, the 6MWT is therefore a better outcome measure to examine the real effect of a walking aid that is experienced by the user in daily life. Implantable PNS resulted in a $23 \%$ improvement of walking speed measured with the 6MWT, whereas the improvement in the control group, while walking with their AFO, was only $3 \%$. Also on the 10 -meter walkway a significant difference was 
found between both groups, in favour of the intervention group. The intervention group showed an improvement of $10 \%$ when the functional electrical stimulation (FES) system was used, while the walking speed in the control group, who used their AFO, did not change during the trial. A questionnaire survey performed by Taylor et al. (17) showed that the use of PNS reduced the effort of walking and improved the confidence of patients while walking. Both aspects can explain the increased walking speed results. An important question is whether the increase in a subjects' walking speed also resulted in a significant gain in their overall functional ability. Definitions, reported by Perry et al. (18) and Burridge et al. (9), can help us to conclude if the differences found in walking speed between both study groups are clinically relevant. Based on the article of Perry et al. (18), de Wit et al. (11) reported in their article that an improvement about $0.2 \mathrm{~m} / \mathrm{s}$ in walking speed can be interpreted as clinically relevant when two walking devices are compared with each other. The definition given by Burridge et al. (9) stated that a $10 \%$ improvement in walking speed between walking with and without walking device is clinically relevant. In accordance to both definitions, both walking speeds calculated from the 6MWT and measured by the 10-meter walkway found in the present study are besides statistically significant also clinically relevant.

In literature, only one other study is reported that also evaluates the effect of an implantable drop foot stimulator, the ACTIGAIT ${ }^{\mathrm{TM}}$, on walking speed (19). In a phase II trial, Burridge et al. found a 19\% improvement in maximum walking speed over time. Although this result is very similar to our findings, the main difference is that Burridge et al. made a comparison with no stimulation, which comparison was also made in their RCT on transcutaneous PNS. In our study a comparison was made with an AFO.

Recently, two other non-randomized studies were performed where the efficacy of a transcutaneous peroneal nerve stimulator device was compared with the efficacy of an AFO (20;21). The first study, performed by Sheffler et al., evaluated functional ambulation by using the modified Emory Functional Ambulation Profile (20). Walking speed was not included as outcome measure. They found that the Odstock Drop Foot Stimulator (ODFS) and an AFO were comparable in their effect on improving functional ambulation as compared to no device. They advised that further study will be necessary, since the study design they used imposed a significant disadvantage on the ODFS trial. All subjects had extensive familiarity with walking with an AFO. However, only 1 day training with the ODFS was received by them, which period is much too 
short to optimally capture the effect of PNS. The second study, performed by Ring et al., did not found a significant difference in gait speed with the neuroprosthesis (21). Because some spatiotemporal parameters measured in their study improved, they concluded that a neuroprosthesis may manage a drop foot more effectively.

Taking together the walking speed results found in our review (chapter 2) and the present RCT (chapter 3), it can be concluded that PNS results in a more improved walking speed compared to an AFO in hemiplegic subjects who are suitable for both PNS and AFO treatment.

\section{Hemiplegic gait pattern}

Studies on the effect of drop foot stimulation on the hemiplegic gait pattern and the compensation strategies used by these patients are scarce. More information about whether PNS has a superior effect on the hemiplegic gait pattern in comparison with the conventional walking devices would be of great help for clinicians to make better recommendations regarding these technical aids. The objective of the present study (chapter 5) was to investigate if an implantable two-channel peroneal nerve stimulator can improve gait performance of stroke survivors with a drop foot and whether these improvements are superior to those attained with conventional walking devices, being an AFO or orthopaedic shoe. Spatiotemporal parameters and hip, knee and ankle kinematics were measured using a three-dimensional video camera system (Vicon®) at baseline and at week 26. PNS seems very effective to provide ankle dorsiflexion during the swing phase of gait and to result in a safe initial contact and loading response in the stance phase. These results are consistent with earlier findings of Ladouceur et al. and Kim et al., who found in incomplete spinal cord injured (SCI) subjects that foot clearance was improved with FES and not with an AFO (22,23). In patients with Multiple Sclerosis (MS), the same findings were reported by Sheffler et al. (24). This adequate pre-positioning is likely to prevent stroke patients from stumbling or falling. No evidence was found in the present study that PNS may trigger hip and knee flexion, which is described in literature $(3,25)$. PNS and a conventional walking aid seem to have a comparable effect on spatiotemporal parameters. The present study results support the use of PNS as treatment option for a drop foot in chronic stroke survivors. 


\section{Physical activity}

Our expectation was that subjects who use PNS would show an increased physical activity, because several studies have suggested that walking with PNS would be more energy efficient, indicated by a reduced physiological cost index (PCI) $(9,26)$. PCI, calculated as the heart rate when walking at a constant speed minus the resting heart rate (beats/min) divided by the walking speed $(\mathrm{m} / \mathrm{min})$, is often used to estimate the effort of walking. Lower values indicate a more efficient gait $(27,28)$. A remarkable finding was that physical activity did not increase as a result of the use of the implantable PNS (chapter 3).

In SCI subjects, it was found that regular stimulation for 1-2 hours a day of their paralyzed muscles can increase fatigue resistance and can strengthen their muscles (2931 ). When subjects are able to walk more efficiently and their muscles will be more fatigue resistant, they should be able to walk for a longer period. Although the walking capacity might be increased in subjects who used the implantable PNS, it seems that they do not change their daily activity pattern during workdays. From personal communication with the implanted subjects it became clear that some of them reported to be more active especially during the weekend, when making trips. In stead of using the wheelchair they are now able to walk all day when using their PNS. However, when setting up the trial, it was decided to exclude the weekend period when measuring subjects' physical activity, because this would hamper making a comparison between the assessments performed before and after 6 months of PNS.

In conclusion, the results do suggest that stroke subjects do not change their daily average walking distance when using FES, whereas the increase in walking speed (chapter 3) allows them to spend even less time in walking.

\section{Health-Related Quality of Life}

The ultimate goal of rehabilitation of stroke survivors is to regain independence and improve quality of life (HRQoL) (32). Regaining the ability to walk is therefore very important. Although many studies examined the clinical effectiveness of PNS on functional outcomes, none of them investigated if PNS also resulted in an improved HRQoL. To our knowledge, this is the first clinical study that focussed on al three domains of the International Classification of Functioning, Disability, and Health (ICF) model of the World Health Organization (33). The goal of the present study (chapter 6) was to examine if implantable PNS improves HRQoL, when compared with an AFO, 
measured by the Short-Form 36 (SF-36) and the Disability Impact Profile (DIP) questionnaire. A significant effect of the implantable peroneal nerve stimulator on the domains physical functioning, general health and the physical component summary score of the SF-36 and on the mobility domain of the DIP questionnaire was found. It can be concluded that PNS seems to be efficient to improve HRQoL, mainly on the domains related to physical functioning. More RCTs that quantify HRQoL will be important to confirm this effect of PNS-assisted ambulation.

When looking at the ICF model, the improved HRQoL is not easily explained by the results found for each separate level of functioning (body functions/ structure level (impairment), activity level (disability) and participation level (handicap)). Stroke subjects who use PNS improve on both body functions/ structure level and activity level, by respectively showing an increased tibialis anterior activity (chapter 4) and the ability to walk faster and for a longer duration (chapter 3). Although they have the capacity, subjects using PNS do not improve on participation level, since they spend less time on walking (chapter 3). An improved HRQoL perception would only be expected when subjects participate more to the community and not when they are less active. A possible explanation for the improved HRQoL results might be the improvement in foot movement caused by the ES, which factor is cited by subjects in the study of Sheffler et al. (20). In comparison with the ankle immobility they experienced when using an $\mathrm{AFO} /$ orthopaedic shoe, this factor could have positively influenced their wellbeing. Another explanation for the increased HRQoL results could have been the novelty effect of the implantable two-channel peroneal nerve stimulator.

\section{Therapeutic effect of PNS}

Besides the orthotic effect, also the presence of a 'therapeutic effect' of PNS was investigated by measuring muscle output of four lower leg muscles during maximal isometric voluntary contractions (MIVC), tibialis anterior (TA) muscle activity during the swing phase of gait and walking speed when the stimulator was not switched on (chapter 4). The therapeutic effect can be defined as the benefit gained following a period of stimulation. 


\section{Maximal isometric voluntary contractions}

In the present RCT, a significantly increased maximum value of the root mean square (RMSmax) of the TA muscle was found. Besides that voluntary control was improved in the stimulated TA muscle, this was also the case for the antagonistic gastrocnemius muscle. This was not expected, since this muscle was not directly stimulated. Stefanovska et al. (34), who evaluated quantitative data on the influence of chronic ES on spasticity in hemiplegic subjects, also found improved MIVC in both the stimulated TA muscle and the antagonistic triceps surae muscle after 6 months of implantable PNS. In addition, chronic ES resulted in a significant decrease in passive resistance and tonic reflex activity in both muscles during the performance of sinusoidal movements of the foot in the sagital plane. According to Stefanovska et al., the absence of tonic activity enabled improved voluntary control in both stimulated muscle groups as well as in the antagonistic group.

\section{TA muscle activity during swing phase}

TA muscle activity was not changed during the swing phase of gait after 6 months of PNS, indicating that no task specific learning for TA muscle activation took place. This finding does not support the Hebbian learning principle, described by Rushton et al. (35). They reported that a possible explanation for the finding that ES in particular is successful in promoting functional recovery might be the unique feature that it activates nerve fibres both orthodromically and antidromically. The antidromic impulse in motor nerve fibres will reach the anterior horn cell (AHC). If the corticospinalanterior horn cell synapse is a Hebb-type modifiable synapse, i.e. one that is strengthened by the coincidence of presynaptic an postsynaptic activity, then FES, combined with coincident voluntary effort through a damaged pyramidal motor system, could help to promote restorative synaptic modifications at AHC level, by this unique adaptive mechanism. Therefore, foot drop stimulation, where stimulation is applied at the time the automatic toe-lift command is generated in the brain, might be in particular successful in promoting functional recovery.

Therefore, in the presence of Hebb-synapses, an increased TA activity during the swing phase of gait after using PNS was expected, since voluntary output is given by the brain to the AHC at the moment the automatic toe-lift command is generated in the brain. 


\section{Walking speed}

Walking speed, both with and without conventional walking aid, was also not improved in the intervention group after they have used PNS for 6 months. Literature is not conclusive. Some studies found a therapeutic effect of lower limb stimulation on walking speed $(4,7,27,36)$, while others did not $(9,37)$. Recently, a meta-analysis was published on the therapeutic effect of PNS on walking speed in stroke survivors (15), resulting in a significant mean improvement of $0.18 \mathrm{~m} / \mathrm{s}$. However, only 3 studies $(4,9,38)$ were entered in the fixed-effects model of which one included subjects in the sub-acute phase after stroke (4). Especially in this study a large effect size was found, whereas relatively small effect sizes were found in the other two studies examining subjects in the chronic stage of recovery. Robbins et al. did not pay any attention if the large effect size in the study who included sub-acute stroke subjects was a response on the FES treatment, if spontaneous recovery took place or if a combination of both was responsible for the increased walking speed results. Since this study had the largest weight in the fixed-effects model, this could have influenced the calculated pooled effect size. The chronic status of our subjects (intervention group: 109 months poststroke; control group: 68 months post-stroke) may be a possible explanation for the fact that no therapeutic effect on walking speed was found after PNS.

\section{Potential mechanisms of recovery}

Although no functional therapeutic effect was found in the present RCT, Sheffler et al. described that long-term plasticity in humans is most likely in activities that are functional relevant and require cognitive input (39). PNS provides a unique opportunity to evaluate motor relearning effects of ES, because ambulation requires a cognitive input and is both important and meaningful to the stroke survivor (39). In contrast, they stated that the prescription of AFOs for dorsiflexion and eversion weakness in hemiparesis may have a negative effect on motor recovery, because residual active movement at the ankle joint is not promoted. However, this assumption made by Sheffler et al. is not supported by the findings of Geboers et al. (40), who found that using an AFO to correct a drop foot after recent peroneal nerve paresis had no significant (negative) effect on restoration of strength. The general decrease of strength values of the healthy side of the patient group compared with the controls may indicate an overall loss of strength caused by less activity. 
Rushton et al. (35) described that there are possibly central as well as peripheral mechanisms which contribute to therapeutic benefit in FES. The peripheral mechanisms of therapeutic effect is by improvement in muscle strength (improving fitness and strength of the remaining motor units), increase in muscle length (improving flexibility and range of motion of affected limb) and reduction of spasticity in the muscle. One of the central mechanisms implicated in mechanisms of therapeutic benefit is the stimulation of cortical reorganization processes, such as unmasking of latent pathways or recruitment/sprouting of new neurons (neuronal plasticity) (41). According to Rushton (35), the central mechanism is more likely to play a role when a therapeutic effect is found in stroke subjects after the use of FES.

To study if a local training effect of the stimulated muscle or a change in motor control (i.e. an increased number of motor units) was responsible for the increased TA muscle activity, the use of high density surface EMG (array) electrodes for investigation of changes in motor unit behaviour (42), or brain imaging techniques, such as positron emission tomography (PET), functional magnetic resonance imaging (fMRI) and transcranial magnetic stimulation (TMS), for examination of changes in motor cortex activation are required in future (43). Recently, Everaert et al. (2009) determined the effect of long-term use of a drop foot stimulator on residual corticospinal connections in stroke subjects by measuring motor-evoked potentials (MEP) from TMS over the motor cortex, MVC and maximum motor wave (Mmax) from stimulating the common peroneal nerve (44). Besides the increased therapeutic effect on walking speed, also the MEP and MVC increased significantly by $50 \%$ and $48 \%$ respectively after using FES, which means that participants had better voluntary control over the TA muscle. Mmax for the TA muscle was also increased, which indicates that the muscle size increased (hypertrophy) caused by training. However, this increases was much smaller and not correlated with the changes in the MEP and MVC. These findings suggest that regular use of a drop foot stimulator strengthens activation of motor cortical areas and their residual descending connections. Increased MEPs were measured at locations adjacent to tissue damaged by the disease process, indicating that these areas may take over to some extent the role of the damaged area. The mechanism for this shift is not known but probably involves coupling of sensory signals from the electrical stimulation and from the walking movements with motor signals. This coupling might lead to Hebbian learning (35), perhaps through long-term potentiation. 
In conclusion, no functional therapeutic effect of implantable PNS was found in the present thesis. Only on peripheral level an improvement was found, no central changes took place.

\section{Implications for clinical practice}

A clinically relevant orthotic effect of the STIMuSTEP ${ }^{\mathrm{TM}}$ on mobility and quality of life was found in a selected group of chronic stroke subjects with a drop foot, when comparing this neuroprosthetic device with the standard of care, an AFO. However, no convincing therapeutic effect of implantable PNS was found in the present RCT.

\section{Transcutaneous versus implantable devices}

When subjects want to use PNS to correct their drop foot, a choice has to be made between using a transcutaneous or implantable stimulator. Nowadays, several transcutaneous drop foot devices are available on the market. The ODFS is probably the most widely used stimulator. Different studies have found that the ODFS resulted in an improved walking speed and a decreased energy expenditure $(9,36)$. Recently, some new transcutaneous stimulators for drop foot have been commercially introduced. In 2006, the WalkAid® was introduced, a transcutaneous stimulator controlled by a tilt sensor attached just below the knee. No clinical studies that examined the effect of this stimulator have been published at the moment. Another new FES neuroprosthesis for the treatment of drop foot was recently developed, the NESS L-300 ${ }^{\mathrm{TM}}$. This stimulator is controlled by a heel switch that communicates wireless with the stimulator. The electrodes are integrated within a semi-rigid orthosis positioned just below the knee, to make it easier for patients to put the system and take off. The idea behind this application is to overcome barriers in the application of non-invasive FES technology for lower limb activation. Ring et al. (21) reported that, compared with an AFO, the NESS L-300 ${ }^{\mathrm{TM}}$ appears to enhance gait asymmetry index and swing time variability during walking and therefore is able to more effectively manage a drop foot.

At present, three implantable peroneal nerve stimulators are available, the STIMuSTEPTM, he ACTIGAIT stimulator and the Neurostep ${ }^{\circledR}$. At the moment, the ACTIGAIT is only tested in a phase II trial and the Neurostep ${ }^{\circledR}$, which is a fully implantable system, is tested for its safety, efficacy and technical performance. With 
respect to function, the eversion component during the swing phase of gait is easier to establish with implantable devices in comparison to transcutaneous devices. Especially for subjects whose mobility is significantly improved by surface stimulation, but who experience problems with electrode placement, painful sensation of stimulation, skin irritation or donning and doffing of the device, an implantable stimulator could be very appropriate. Also for drop foot subjects who want to use PNS over a long period, an implantable stimulator offers greater comfort than a transcutaneous stimulator. Taylor et al. reported that, unless that subjects were satisfied about the use of the ODFS device, two thirds of them would consider an implanted system instead (45). Implantable stimulators are not indicated when reaching a therapeutic effect is the main goal, because of the surgery procedure that is involved.

\section{Treatment preferences of stroke subjects}

Although using an AFO or peroneal nerve stimulator may be comparable in their effect on improving functional ambulation in stroke subjects with suitability for both devices, recent studies reported that subjects prefer PNS $(20,21,46)$. Subjects who preferred PNS cited positive factors including improvements in muscle movement, gait pattern and strength (20). Van Til et al. investigated patients' preferences with regard to treatment process and outcome in ankle-foot impairment in patients with central neurological disorder, by using a discrete choice experiment (47). The results of their study indicate that both surgery and neuroprosthetic devices are accepted by patients and therefore have potential in the treatment of ankle-foot impairment.

\section{Limitations for widespread use}

In 1961, PNS was already invented by Liberson et al. (7). Remarkably, the number of subjects using PNS at home and in clinical settings is still limited. Different reasons can be responsible for this limited clinical implementation. First, technology problems in the past could have played a role. Present FES technology has advanced considerably, so that clinicians may prescribe neuroprosthetic transcutaneous ES devices more often in future. For example the hybrid orthosis of the NESS L300 drop foot stimulator, which includes two electrodes, is designed to enable accurate and repeatable placement. Especially for subjects who are not able to use both hands, like most stroke subjects, and for subjects who experience difficulty with electrode placement, this stimulator is much 
easier to put on and take off when compared to the current drop foot stimulators. Second, it took a long time before peroneal nerve systems were approved by the Food and Drug Administration (FDA). Nowadays, the ODFS, the Walkaide System and the Ness L300 have FDA approval, which can probably result in a broader clinical acceptance. Third, the training aspect did not get enough attention. To increase the use of this technology in future, proper training of therapists, users and their carers on the use of PNS is very important to ensure that the subject is obtaining maximum possible benefit from the stimulation device. Fourth, more information regarding determinants that predict a clear benefit of PNS is needed. Some general in- and exclusion criteria for the use of FES are already known. One of the most important selection criteria used in the present study that determined the success of implantable PNS was the range of motion (ROM) of the affected ankle joint. A better ROM was associated with an easier lifting of the foot by the stimulator. Also motivation of subjects seemed to be a very important factor. Further research is needed to identify these determinants. Finally, funding of PNS by health insurance companies needs more attention, so that more drop foot subjects will have access to this technology. In The Netherlands this is an important reason that still a limited number of subjects are using PNS. In comparison with an AFO, which costs are about 250 euro for a non-custom made AFO, the costs of a drop foot stimulator are much higher, varying between 1,000 (ODFS) and 6,000 euro (NESS L300) for transcutaneous devices, and 11,000 euro for the implantable STIMUSTEP ${ }^{\text {TM }}$. So the question is if the advantages of using an implantable device in comparison with an AFO, which are an increased walking speed and HRQoL, are worth to pay 11,000 euro. For some of the included subjects this will definitely be the case. However, most subjects will not be able to pay this amount of money by themselves, so support of health insurance companies will be crucial to achieve that FES-assisted walking will become an integral part of stroke rehabilitation in future. Economic evaluations, by means of a cost-utility analysis, are therefore advised to obtain a good understanding about which drop foot treatment is the most efficient one.

\section{Further topics of study}

To our knowledge, this was the first RCT on (implantable) PNS where a comparison was made between ES and conventional splinting. When looking at the literature about PNS, most of the studies were non-randomized and involved small numbers of subjects. 
Therefore large-scale randomized trials will be required for getting wider clinical acceptance for this therapy. Multi-centre trials will probably be the solution to include more rehabilitation subjects.

In addition, the STIMUSTEPTM is only tested in a select group of chronic stroke survivors with a drop foot. Because of this selection, the preference of PNS, when compared to an $\mathrm{AFO}$, with regard to improving impaired body functions, is not applicable to all stroke subjects who use an AFO. Future studies might investigate the generalizability of the results, also to other subject categories with upper motoneuron drop foot problems, like MS and incomplete SCI subjects. Some first studies already showed positive results in these patient groups $(23,36,46,48)$, but more studies are needed in future. Randomized trials that make a comparison between the neuroprosthetic effect of a peroneal nerve stimulator with the orthotic effect of an AFO have to be performed.

Less is also known about the durability of the implant, since the focus in the present trial was on the immediate effects of PNS. In the study period of 6 months, only 1 out of 14 subjects dropped out at week 12, because of a defective implant. In the meanwhile, more information about the long-term use of the implant is obtained in a nonsystematic way. Of the remaining 13 implanted subjects, the implant is removed in 1 other subject. This patient had an abscess at the location of the scar, which harmed the implant. Recently another subject reported problems with his implant, as result of a fall on his leg at the location of the implant. Since this patient walks much easier and further with PNS, he prefers to use a transcutaneous stimulator at the moment, in stead of his AFO. More information about the durability of the implantable part of the PNS is needed to decide if the implant needs to be improved in future.

In the meanwhile, some technological improvements to the implant have already taken place by Finetech Medical Ltd.. Firstly, a slight change is made to the way the electrode is connected to the lead, so that the wire within the electrode body is less sensitive to fracture. Secondly, besides an implant with epineural electrodes, also an implant with cuff electrodes is designed and manufactured. In this way each neurosurgeon can choose the implantation technique in which he is most experienced. An advantage of using cuff electrodes is that, after surgery, one is allowed to selectively control the muscles that are stimulated, while with epineural electrodes the control of the different 
muscles is fixed by the position of the electrodes and one can only adjust the stimulation current.

Besides stimulating the dorsalfexors and evertors, also other muscles like the hamstring or calf muscles can be stimulated to enable knee flexion or push-off in terminal stance, which can improve the hemiplegic gait. For the activation of multiple muscles a more advanced implanted system has to be developed in future. Also combinations of different interventions, such as combining ES with botox injections, surgery or robot devices have to be studied to improve the hemiplegic gait pattern. 


\section{References}

1. IJzerman MJ, Renzenbrink GJ, Geurts AC. Neuromuscular stimulation after stroke: from technology to clinical deployment. Expert Rev Neurother 2009 Apr;9(4):541-52.

2. Waters RL, McNeal D, Perry J. Experimental correction of footdrop by electrical stimulation of the peroneal nerve. J Bone Joint Surg Am 1975 Dec;57(8):1047-54.

3. Merletti R, Andina A, Galante M, Furlan I. Clinical experience of electronic peroneal stimulators in 50 hemiparetic patients. Scand J Rehabil Med 1979;11(3):111-21.

4. Bogataj U, Gros N, Kljajic M, Acimovic R, Malezic M. The rehabilitation of gait in patients with hemiplegia: a comparison between conventional therapy and multichannel functional electrical stimulation therapy. Phys Ther 1995 Jun;75(6):490-502.

5. Burridge J, Taylor P, Hagan S, Swain I. Experience of clinical use of the Odstock dropped foot stimulator. Artif Organs 1997 Mar;21(3):254-60.

6. Burridge JH, Swain ID, Taylor PN. Functional electrical stimulation: a review of the literature published on common peroneal nerve stimulation for the correction of dropped foot. Reviews in Clinical Gerontology 1998;8:155-61.

7. Liberson WT, Homquest HJ, Scot D, Dow M. Functional electrotherapy: stimulation of the peroneal nerve synchronized with the swing phase of the gait of hemiplegic patients. Arch Phys Med Rehabil 1961 Feb;42:101-5.

8. Waters RL, McNeal DR, Faloon W, Clifford B. Functional electrical stimulation of the peroneal nerve for hemiplegia. Long-term clinical follow-up. J Bone Joint Surg Am 1985 Jun;67(5):792-3.

9. Burridge JH, Taylor PN, Hagan SA, Wood DE, Swain ID. The effects of common peroneal stimulation on the effort and speed of walking: a randomized controlled trial with chronic hemiplegic patients. Clin Rehabil 1997 Aug;11(3):201-10.

10. Chae J. Neuromuscular electrical stimulation for motor relearning in hemiparesis. Phys Med Rehabil Clin N Am 2003 Feb;14(1 Suppl):S93-109.

11. de Wit DC, Buurke JH, Nijlant JM, Ijzerman MJ, Hermens HJ. The effect of an ankle-foot orthosis on walking ability in chronic stroke patients: a randomized controlled trial. Clin Rehabil 2004 Aug;18(5):550-7.

12. Richards CL, Olney S.Hemiparetic gait following stroke. Part II: Recovery and physical therapy 1996;4:149-62.

13. Wade DT. Measurement in Neurological Rehabilitation. Oxford: Oxford University Press: 1992.

14. Kottink AI, Hermens HJ, Nene AV, Tenniglo MJ, van der Aa HE, Buschman HP, Ijzerman MJ. A randomized controlled trial of an implantable 2-channel peroneal nerve stimulator on walking speed and activity in poststroke hemiplegia. Arch Phys Med Rehabil 2007 Aug;88(8):971-8. 
15. Robbins SM, Houghton PE, Woodbury MG, Brown JL. The therapeutic effect of functional and transcutaneous electric stimulation on improving gait speed in stroke patients: a metaanalysis. Arch Phys Med Rehabil 2006 Jun;87(6):853-9.

16. Guyatt GH, Sullivan MJ, Thompson PJ, Fallen EL, Pugsley SO, Taylor DW, Berman LB. The 6-minute walk: a new measure of exercise capacity in patients with chronic heart failure. Can Med Assoc J 1985 Apr 15;132(8):919-23.

17. Taylor PN, Burridge JH, Dunkerley AL, Lamb A, Wood DE, Norton JA, Swain ID. Patients' perceptions of the Odstock Dropped Foot Stimulator (ODFS). Clin Rehabil 1999 Oct;13(5):439-46.

18. Perry J, Garrett M, Gronley JK, Mulroy SJ. Classification of walking handicap in the stroke population. Stroke 1995 Jun;26(6):982-9.

19. Burridge JH, Haugland M, Larsen B, Pickering RM, Svaneborg N, Iversen HK, Christensen PB, Haase J, Brennum J, Sinkjaer T. Phase II trial to evaluate the ActiGait implanted dropfoot stimulator in established hemiplegia. J Rehabil Med 2007 Apr;39(3):212-8.

20. Sheffler LR, Hennessey MT, Naples GG, Chae J. Peroneal nerve stimulation versus an ankle foot orthosis for correction of footdrop in stroke: impact on functional ambulation. Neurorehabil Neural Repair 2006 Sep;20(3):355-60.

21. Ring H, Treger I, Gruendlinger L, Hausdorff JM. Neuroprosthesis for footdrop compared with an ankle-foot orthosis: effects on postural control during walking. J Stroke Cerebrovasc Dis 2009 Jan;18(1):41-7.

22. Ladouceur M, Barbeau H. Functional electrical stimulation-assisted walking for persons with incomplete spinal injuries: changes in the kinematics and physiological cost of overground walking. Scand J Rehabil Med 2000 Jun;32(2):72-9.

23. Kim CM, Eng JJ, Whittaker MW. Effects of a simple functional electric system and/or a hinged ankle-foot orthosis on walking in persons with incomplete spinal cord injury. Arch Phys Med Rehabil 2004 Oct;85(10):1718-23.

24. Sheffler LR, Bailey SN, Chae J. Spatiotemporal and kinematic effect of peroneal nerve stimulation versus an ankle-foot orthosis in patients with multiple sclerosis: a case series. PM R 2009 Jul;1(7):604-11.

25. Burridge J, Taylor P, Hagan S, Wood D, Swain I. The effect of common peroneal nerve stimulation on quadriceps spasticity in hemiplegia. Physiotherapy 1997;83:82-9.

26. Weber DJ, Stein RB, Chan KM, Loeb G, Richmond F, Rolf R, James K, Chong SL. BIONic WalkAide for correcting foot drop. IEEE Trans Neural Syst Rehabil Eng 2005 Jun;13(2):242-6.

27. Stein RB, Chong S, Everaert DG, Rolf R, Thompson AK, Whittaker M, Robertson J, Fung J, Preuss R, Momose K, et al. A multicenter trial of a footdrop stimulator controlled by a tilt sensor. Neurorehabil Neural Repair 2006 Sep;20(3):371-9. 
28. MacGregor J. The evaluation of patient performance using long-term ambulatory monitoring technique in the domiciliary environment. Physiotherapy 1981 Feb 10;67(2):30-3.

29. Belanger M, Stein RB, Wheeler GD, Gordon T, Leduc B. Electrical stimulation: can it increase muscle strength and reverse osteopenia in spinal cord injured individuals? Arch Phys Med Rehabil 2000 Aug;81(8):1090-8.

30. Rodgers MM, Glaser RM, Figoni SF, Hooker SP, Ezenwa BN, Collins SR, Mathews T, Suryaprasad AG, Gupta SC. Musculoskeletal responses of spinal cord injured individuals to functional neuromuscular stimulation-induced knee extension exercise training. J Rehabil Res Dev 1991 Fall;28(4):19-26.

31. Stein RB, Gordon T, Jefferson J, Sharfenberger A, Yang JF, de Zepetnek JT, Belanger M. Optimal stimulation of paralyzed muscle after human spinal cord injury. J Appl Physiol 1992 Apr;72(4):1393-400.

32. Kwa VI, Limburg M, de Haan RJ. The role of cognitive impairment in the quality of life after ischaemic stroke. J Neurol 1996 Aug;243(8):599-604.

33. Geneva World Health Organization. WHO: International Classification of Functioning, Disability and Health (ICF)2001.

34. Stefanovska A, Gros N, Vodovnik L, Rebersek S, Acimovic-Janezic R. Chronic electrical stimulation for the modification of spasticity in hemiplegic patients. Scand J Rehabil Med Suppl 1988;17:115-21.

35. Rushton DN. Functional electrical stimulation and rehabilitation--an hypothesis. Med Eng Phys 2003 Jan;25(1):75-8.

36. Taylor PN, Burridge JH, Dunkerley AL, Wood DE, Norton JA, Singleton C, Swain ID. Clinical use of the Odstock dropped foot stimulator: its effect on the speed and effort of walking. Arch Phys Med Rehabil 1999 Dec;80(12):1577-83.

37. Granat MH, Maxwell DJ, Ferguson AC, Lees KR, Barbenel JC. Peroneal stimulator; evaluation for the correction of spastic drop foot in hemiplegia. Arch Phys Med Rehabil 1996 Jan;77(1):19-24.

38. Alon G RH. Gait and hand function enhancement following training with a multi-segment hybrid-orthosis stimulation system in stroke patients. J Stroke Cerebrovasc Dis 2003;12:209-16.

39. Sheffler LR, Hennessey MT, Naples GG, Chae J. Improvement in functional ambulation as a therapeutic effect of peroneal nerve stimulation in hemiplegia: two case reports. Neurorehabil Neural Repair 2007 Jul-2007 Aug 31;21(4):366-9.

40. Geboers JF, Janssen-Potten YJ, Seelen HA, Spaans F, Drost MR. Evaluation of effect of ankle-foot orthosis use on strength restoration of paretic dorsiflexors. Arch Phys Med Rehabil 2001 Jun;82(6):856-60. 
41. Calautti C, Baron JC. Functional neuroimaging studies of motor recovery after stroke in adults: a review. Stroke 2003 Jun;34(6):1553-66.

42. Kallenberg LA, Hermens HJ. Motor unit properties of biceps brachii in chronic stroke patients assessed with high-density surface EMG. Muscle Nerve 2009 Feb;39(2):177-85.

43. Cramer SC, Bastings EP. Mapping clinically relevant plasticity after stroke. Neuropharmacology 2000 Mar 3;39(5):842-51.

44. Everaert DG, Thompson AK, Chong SL, Stein RB. Does Functional Electrical Stimulation for Foot Drop Strengthen Corticospinal Connections? Neurorehabil Neural Repair 2009 Oct 27.

45. Taylor PN, Mann G, Wood DE, Hobby J. Pilot study to evaluate the safety and efficacy of an implanted dropped foot stimulator; International Functional Electrical Stimulation Society (IFESS) Conference 2003; Maroochydore, Australia.

46. Sheffler LR, Hennessey MT, Knutson JS, Chae J. Neuroprosthetic effect of peroneal nerve stimulation in multiple sclerosis: a preliminary study. Arch Phys Med Rehabil 2009 Feb;90(2):362-5.

47. van Til JA, Dolan JG, Stiggelbout AM, IJzerman MJ. Conjoint analysis and analytic hierarchy process to determine preferences for health interventions. Annual meeting of the society for Medical Decision Making 2009; California USA.

48. Paul L, Rafferty D, Young S, Miller L, Mattison P, McFadyen A. The effect of functional electrical stimulation on the physiological cost of gait in people with multiple sclerosis. Mult Scler 2008 Aug;14(7):954-61. 


\section{Summary}

A drop foot is a motor impairment which is commonly seen in stroke survivors. The abnormal, inefficient and unsafe walking pattern hinders patients to perform their activities of daily living, resulting in an impaired quality of life. In the past several solutions have been developed for the treatment of a drop foot. Functional electrical stimulation (FES) of the peroneal nerve is one of the treatment options that can be used to correct a drop foot. Although this concept has existed for more than 40 years, the use of FES is not widespread and the total number of patients being treated remains quite small. Several reasons can be responsible for this finding, such as technical limitations and unfamiliarity with FES in many countries. In addition there is still no hard evidence for the positive clinical effects of this treatment. Until now only 1 randomized controlled trial (RCT) was performed with several limitations. An interesting aspect that has not been clarified yet is the additional value of peroneal nerve stimulation in comparison with the treatment that is normally given to stroke survivors, i.e. a passive walking device.

The aim of the present thesis was to attribute to a more evidence based stroke rehabilitation regarding the correction of a drop foot by FES. A RCT was performed to evaluate the effect of an implantable two-channel peroneal nerve stimulator on impairment, disability and quality of life in comparison with the effect of the conventional treatment given to stroke survivors with a drop foot, in most cases an ankle-foot orthosis (AFO).

First of all a systematic review is performed to identify trials that investigated the orthotic effect of FES on walking in stroke survivors with a drop foot, which is described in chapter 2. Studies that measured walking speed were pooled and a pooled difference including confidence interval was calculated. Eight studies were included in the review, of which one was a RCT. Methodological score ranged from 8 to 18 out of 19. Six of the eight studies measured walking speed. Four studies included chronic stroke patients and in two studies acute, sub-acute and chronic patients were included. The pooled improvement in walking speed was $0.13 \mathrm{~m} / \mathrm{s}(0.07-0.2)$ or $38 \%(22.18-53.8)$. Therefore FES seems to have a positive orthotic effect on walking speed.

Chapter 3 focuses on the orthotic effect of the implantable two-channel peroneal nerve stimulator on walking speed and daily activities. Twenty-nine stroke survivors with 
chronic hemiplegia and drop foot who fulfilled the predefined inclusion and exclusion criteria were included. Fourteen subjects received the implantable stimulator and 15 subjects continued using their conventional walking device, consisting of an AFO, orthopedic shoe, or no device. Walking speed was assessed by both the 6-minute walk test (6MWT) and the 10-meter walk test. An activity monitoring system was used to measure physical activity, resulting in percentage time spent on stepping, standing, and sitting/lying. Implantable FES resulted in a $23 \%$ improvement of walking speed measured by the $6 \mathrm{MWT}$, whereas the improvement in the control group was only $3 \%$. Comfortable walking speed measured on a $10-\mathrm{m}$ walkway was also significantly improved in favor of FES. Both walking speed results seemed to be clinical relevant in accordance with the scientific literature. Concerning the activity monitoring data, both the intervention and control group showed a deterioration in time spent on stepping, the FES group a bit more than the control group. However, no significant difference was found between both groups.

Although the implantable two-channel peroneal nerve stimulator resulted in a significant and clinically relevant increase in walking speed, the results do suggest that the average walking distance did not change by applying the stimulator.

In chapter 4 the question is addressed whether there is a therapeutic effect in case of an implantable two-channel peroneal nerve stimulator. The same study population was measured at baseline and at weeks 4, 8, 12 and 26 in the gait laboratory. The maximum value of the root mean square (RMSmax) of the tibialis anterior (TA), peroneus longus (PL), gastrocnemius (GS) and soleus (SL) muscles of the affected leg was measured in both flexed and extended knee. Also walking speed and the muscle activity of the TA muscle of the affected leg during the swing phase of gait were measured. A significantly higher RMSmax of the TA muscle with extended knee and a significantly increased RMSmax of the GS muscle with both flexed and extended knees was found after using FES. No change in walking speed was found when the stimulator was not switched on. Also no increase in TA muscle activity of the affected leg was found during the swing phase of gait after use of the peroneal nerve stimulator.

Functionally no therapeutic effect of implantable peroneal nerve stimulation was found. However, the significantly increased voluntary muscle output of the TA and GS muscles after the use of FES suggests that there was a certain extent of plasticity in the subjects in this study. 
Chapter 5 describes whether a superior value of implantable PNS over the more commonly used conventional walking aids (AFO and orthopaedic shoes) on the hemiplegic gait pattern was present. Twenty-three chronic stroke survivors with drop foot fulfilled the predefined inclusion and exclusion criteria and were included in the present RCT. All subjects were measured on spatiotemporal parameters of both the paretic and non-paretic limb and hip, knee and ankle kinematics of the paretic limb using a three-dimensional video camera system (Vicon $®)$ at baseline and after a followup period of 6 months. Both peroneal nerve stimulator and conventional walking aid seem to have rather comparable effects on the spatiotemporal parameters of the hemiplegic gait. A significant reduction in both ankle plantarflexion angles during swing and at heel strike were found as a result of PNS in the present study. No evidence was found that PNS may trigger hip and knee flexion.

The findings of the present study suggest that PNS, in comparison with using a conventional walking aid, seems more effective to provide ankle dorsiflexion during swing and results in a safer initial loading response during stance, which will probably contribute to reducing the risk of stumbling or falling.

Chapter 6 addresses the question whether the treatment of a drop foot by means of an implantable two-channel peroneal nerve stimulator improves patients' health-related quality of life. HRQoL was assessed in two different ways: (1) by taking descriptive measures, i.e. the Short Form-36 (SF-36; generic measure) and the Disability Impact Profile (DIP; specific measure); and (2) by obtaining preference-based utilities both measured with the Time Trade Off (TTO; direct way) and by calculating them from the Euroqol-5D (EQ-5D) and SF-36. The first goal was to examine if the implantable twochannel peroneal nerve stimulator improves HRQoL. The second goal was to investigate the responsiveness of the included preference-based utility measures to detect clinically important changes. The third and last goal was to examine if a relation was present between different preference-based utility outcomes, measured both in a direct (TTO) and indirect way (EQ-5D and SF-36). A significant positive effect of the implantable device was found on the physical functioning domain, the general health domain and the physical component summary score of the SF-36. For the DIP a significant improvement was found on the domains mobility, self-care and psychological status in the intervention group. Regarding the preference-based utility measures, a significant effect was found for the utility index calculated from the EQ-5D. 
In conclusion, the implantable two-channel peroneal nerve stimulator seems to be efficient to improve HRQoL, mainly the domains related to physical functioning. Regarding the responsiveness, both the EQ-5D and SF-36 were in an equal way able to detect changes that are meaningful or clinically important. There was also a relation present between the utility indexes calculated from the EQ-5D and SF-36.

In the final chapter, chapter 7 , the findings of the present thesis are integrated and discussed and recommendations for future research are proposed. Summarizing, the results of this thesis indicate that the STIMUSTEPTM is a clinical effective device in a selected group of chronic stroke subjects with a drop foot. When comparing this neuroprosthetic device with the standard of care, an AFO or orthopaedic shoes, a clinically relevant orthotic effect on mobility, ankle kinematics and quality of life was found. However, no convincing therapeutic effect of PNS was found in the present RCT. 


\section{Samenvatting}

Een sleepvoet is een motorische aandoening welke vaak wordt waargenomen bij CVA patiënten. Deze aandoening resulteert in een afwijkend, inefficiënt en onveilig looppatroon welke patiënten hindert de alledaagse activiteiten uit te voeren, resulterend in een verminderde kwaliteit van leven. In het verleden zijn verschillende therapieën ontwikkelt ter behandeling van een sleepvoet. Functionele elektrische stimulatie (FES) van de peroneus zenuw is een van de mogelijke behandelmogelijkheden ter correctie van de sleepvoet. Alhoewel FES al meer dan 40 jaar bestaat, wordt het nog steeds niet wereldwijd toegepast en blijft het aantal behandelde patiënten relatief klein. Meerdere redenen kunnen hiervoor verantwoordelijk zijn, zoals technische beperkingen en de onbekendheid omtrent het gebruik van FES in vele landen. Tevens is er nog steeds geen hard bewijs voor de positieve klinische effecten van deze behandeling. Tot op heden is 1 gerandomiseerde klinische studie uitgevoerd door Burridge (1997), welke tevens werd gekenmerkt door verschillende beperkingen. Een interessant aspect welke tot op heden niet is onderzocht is welke extra waarde een peroneus stimulatie systeem heeft ten opzichte van de behandeling welke in de dagelijkse praktijk wordt voorgeschreven aan CVA patiënten, namelijk een passieve orthese.

Het doel van dit proefschrift is om bij te dragen aan een meer evidence based revalidatieprogramma voor CVA patiënten met betrekking tot de behandeling van een sleepvoet middels FES. Een gerandomiseerde gecontroleerde studie (RCT) is uitgevoerd waarin het effect van een implanteerbare twee-kanaals peroneus stimulator, de STIMUSTEP ${ }^{\text {TM }}$, op stoornisniveau, beperkingniveau en kwaliteit van leven werd geëvalueerd in vergelijking met het effect van de conventionele behandeling welke aan CVA patiënten met een sleepvoet wordt voorgeschreven, welke in de meeste gevallen een enkel-voet orthese (EVO) is.

Ten eerste is een systematische review uitgevoerd waarin trials zijn geïncludeerd welke het orthetische effect van FES op het lopen bij CVA patiënten met een sleepvoet hebben onderzocht, welke resultaten staan beschreven in hoofdstuk 2. Acht studies werden geïncludeerd in de review, waarin 1 studie een RCT was. De methodologische score varieerde van 8 tot 18 op een totaal van 19 punten. Zes van deze 8 studies hadden loopsnelheid als uitkomstmaat. Vier van deze studies includeerden chronische CVA patiënten en in 2 studies waren zowel acute, subacute en chronische CVA patiënten 
geïncludeerd. De data van 2 van de 6 studies welke loopsnelheid als uitkomstparameter hadden toegevoegd konden niet worden gebruikt voor de berekening van de gepoolde loopsnelheid, aangezien te weinig data hiervoor beschikbaar was. Uit het poolen van de resultaten van de overige 4 studies kwam een gepoolde verbetering in loopsnelheid van $0.13 \mathrm{~m} / \mathrm{s}(0.07-0.2)$ of $38 \%$ (22.18-53.8) naar voren. De toepassing van FES lijkt dus een positief orthetisch effect op loopsnelheid te hebben.

In hoofdstuk 3 ligt de focus op het orthetische effect van de STIMuSTEP ${ }^{\mathrm{TM}}$ op loopsnelheid en het dagelijkse activiteitenpatroon. Negenentwintig CVA patiënten met chronische hemiplegie en een sleepvoet welke voldeden aan de opgestelde in- en exclusiecriteria werden geïncludeerd in de studie. Veertien van hen ontvingen de geïmplanteerde stimulator en 15 van hen continueerden het gebruik van hun voorgeschreven hulpmiddel tijdens de baseline meting, bestaande uit een EVO, een orthopedische schoen of geen hulpmiddel. Loopsnelheid werd gemeten middels de 6 minuten looptest en de 10 meter looptest. Een activiteitenmeter werd gebruikt om de fysieke activiteit te meten, resulterend in het percentage tijd van de dag waarin werd gelopen, gestaan of gezeten/gelegen. Het gebruik van de implanteerbare peroneus stimulator resulteerde in een toename in loopsnelheid van 23\% gemeten met de 6 minuten looptest, terwijl de loopsnelheid in de controle groep maar 3\% verbeterde. De comfortabele loopsnelheid gemeten met behulp van de 10 meter looptest was ook significant verbeterd in de FES groep. De verbetering in loopsnelheid gemeten met zowel de 6 minuten looptest als de 10 meter looptest was naast significant ook klinisch relevant volgens de criteria welke zijn beschreven in de wetenschappelijke literatuur.

Met betrekking tot het activiteitenpatroon kwam naar voren dat zowel de interventie als controle groep een afname liet zien in het percentage tijd dat men spendeerde aan lopen. Echter, er werd geen significant verschil tussen beide groepen gevonden. Ondanks het feit dat veel patiënten aangaven dat de STIMUSTEPTM het lopen vergemakkelijkt, lijkt het erop dat deze patiëntengroep hetzelfde activiteitenpatroon is blijven uitvoeren in vergelijking met voor de operatie. Aangezien het uitvoeren van deze activiteiten gepaard gaat met een toegenomen loopsnelheid is het percentage tijd dat zij spendeerden aan lopen afgenomen.

Hoewel het gebruik van de STIMuSTEPTM resulteerde in een significante en klinisch relevante toename in loopsnelheid, suggereren de resultaten dat de gemiddelde loopafstand niet is veranderd als gevolg van het gebruik van de stimulator. 
Hoofdstuk 4 richt zich op de vraag of het gebruik van de STIMuSTEPTM resulteert in een therapeutisch effect. Hiermee wordt bedoeld of er na een periode van FES gebruik een positief effect aanwezig blijft op het moment dat de stimulator niet wordt gebruikt. Dezelfde studiepopulatie werd hiervoor gebruikt en de metingen vonden plaats tijdens baseline en 4, 8, 12 en 26 weken na de operatie. De maximale spieractiviteit van de tibialis anterior (TA), peroneus longus (PL), gastrocnemius (GS) en de soleus (SL) spier aan de aangedane zijde werd gemeten met behulp van oppervlakte electromyografie (sEMG). Deze meting vond plaats terwijl de positie van de knie zich in flexie en extensie begaf. Daarnaast werd gekeken naar het therapeutische effect van peroneus stimulatie op de loopsnelheid en werd gekeken naar het effect van een periode FES gebruik op de mate van spieractiviteit van de TA spier van het aangedane been tijdens de zwaaifase van het lopen. Uit de resultaten kwam een significante toename in spieractiviteit van de TA spier naar voren terwijl de knie zich in een extensie positie begaf. Daarnaast werd een significant toegenomen spieractiviteit van de GS spier gevonden in beide knieposities na het gebruik van FES. Er werd geen verandering in loopsnelheid waargenomen wanneer geen gebruik werd gemaakt van de stimulator. Tevens werd geen toename in TA activiteit van het aangedane been gevonden tijdens de zwaaifase van het lopen na gebruik van de stimulator.

Concluderend kan gesteld worden dat geen functioneel therapeutisch effect van de STIMUSTEPTM is gevonden. Echter, de significant toegenomen vrijwillige spieractiviteit van zowel de TA en GS spier na het gebruik van FES suggereert dat er wel sprake is van een bepaalde mate van plasticiteit in de huidige studiepopulatie.

Hoofdstuk 5 beschrijft de invloed van de STIMuSTEPTM op de spatiotemporele parameters van zowel het aangedane als niet-aangedane been en de heup, knie en enkel kinematica van het aangedane been tijdens het lopen. Hierbij werd gekeken of FES een toegevoegde waarde heeft ten opzichte van de conventionele hulpmiddelen welke gebruikt worden als behandelmethode voor een sleepvoet, zoals een AFO of orthopedische schoenen. Vanuit de literatuur is bekend dat peroneus stimulatie naast een toegenomen dorsaalflexie van het enkelgewricht ook kan resulteren in een toegenomen flexie van het heup- en kniegewricht. Peroneus stimulatie zou dus een positieve invloed kunnen hebben op het flexiepatroon welke nodig is voor een goede voet clearance tijdens de zwaaifase van het lopen. Hierdoor zou een normaler looppatroon moeten ontstaan welke de noodzaak tot compensatie strategieën 
verminderd, resulterend in een efficiënter gebruik van de heup- en kniespieren. De 2 metingen, welke plaatsvonden tijdens baseline en na 6 maanden gebruik van de peroneus stimulator, bestonden uit het plaatsen van 17 reflecterende markers op het onderlichaam van de proefpersoon. Met behulp van een 3-dimensionaal Vicon ${ }^{\circledR}$ systeem werd het looppatroon van zowel het paretische als niet-paretische been vastgelegd. De controle groep bestond in totaal uit 13 patiënten, terwijl de interventie groep uit 10 patiënten bestond. Uit de resultaten kwam naar voren dat beide behandelmethoden een vergelijkbaar effect hebben op de spatiotemporele parameters van het hemiplegische looppatroon. Daarnaast werd een significante afname gevonden in zowel de plantarflexie hoek van de enkel tijdens de zwaaifase als tijdens de landing van de voet tijdens het gebruik van de implanteerbare peroneus stimulator. Echter, tijdens het gebruik van peroneus stimulatie werd geen toename in heup- en knieflexie gevonden.

Peroneus stimulatie resulteert in een toename van de dorsaalflexie hoek van het enkelgewricht tijdens de zwaaifase van het lopen en zorgt voor een veiliger landing van de voet tijdens de standfase in vergelijking met de conventionele hulpmiddelen. Hierdoor zal het risico op struikelen of vallen waarschijnlijk afnemen.

In hoofdstuk 6 wordt aandacht besteed aan het effect van de FES behandeling ter correctie van een sleepvoet op de kwaliteit van leven van de deelnemende patiënten. De gezondheidsgerelateerde kwaliteit van leven werd gemeten op 2 verschillende manieren: (1) door het afnemen van beschrijvende vragenlijsten, zoals de Short-Form 36 (SF-36: generiek meetinstrument) en de Disability Impact Profile (DIP: ziektespecifiek meetinstrument); en (2) door het verkrijgen van utiliteiten gemeten met zowel de Time Trade Off (TTO) als het berekenen van utiliteiten uit de Euroqol-5D (EQ-5D) en SF-36. Het primaire doel van de studie was te onderzoeken of de STIMUSTEP ${ }^{\mathrm{TM}}$ resulteerde in een verbetering van de kwaliteit van leven. Het tweede doel was het onderzoeken van de interne en externe responsiviteit van de toegepaste utiliteitsvragenlijsten en het derde en laatste doel bestond uit het onderzoeken of er een relatie aanwezig was tussen de verschillende maten welke gebruikt zijn om utiliteit te meten. Uit de resultaten kwam naar voren dat de peroneus stimulator een significant positief effect had op de domeinen fysiek functioneren, algemene gezondheid en de fysieke component score van de SF-36. Tevens werd in de FES groep een significante verbetering gevonden op de domeinen mobiliteit, zelfzorg en psychologische status van 
de DIP. Met betrekking tot de utiliteitsmaten werd alleen een significant effect gevonden op de utiliteitsindex welke was berekend vanuit de EQ-5D. Zowel de EQ-5D als de SF-36 waren de meest responsieve utiliteitsmaat en de hieruit voortvloeiende utiliteitsindexen waren gerelateerd aan elkaar.

Concluderend kan gesteld worden dat de STIMuSTEPTM een positief effect lijkt te hebben op de gezondheidsgerelateerde kwaliteit van leven, met name op de domeinen welke gerelateerd waren aan fysiek functioneren.

In het laatste hoofdstuk, hoofdstuk 7, zijn de verschillende bevindingen uit dit proefschrift geïntegreerd en bediscussieerd en aanbevelingen voor vervolgonderzoek zijn gedaan. Samenvattend komt uit de resultaten naar voren dat de STIMuSTEP ${ }^{\mathrm{TM}}$ een klinisch effectief systeem is voor een geselecteerde groep CVA patiënten met een sleepvoet. Wanneer deze neuroprothese wordt vergeleken met de gebruikelijke behandelmethode, welke een spalk betreft in de meeste gevallen, wordt een klinisch relevant orthetisch effect van de neuroprothese op mobiliteit, enkel kinematica en kwaliteit van leven gevonden. Echter, er is geen wetenschappelijk bewijs gevonden voor de aanwezigheid van een therapeutisch effect na het gebruik van de implanteerbare twee-kanaals peroneus stimulator. 



\section{Dankwoord}

Eindelijk is het dan zover. Ik ben toegekomen aan het schrijven van mijn dankwoord! Hiermee sluit ik een voor mij belangrijke periode af. Dat geeft een heel goed gevoel, maar ook een gevoel van dankbaarheid, omdat velen een bijdrage hebben geleverd aan mijn promotie. Bij deze wil ik hen graag persoonlijk noemen en bedanken.

Prof. dr. ir. Hermens, beste Hermie, jou wil ik bedanken voor de kans die je mij hebt gegeven om als afgestudeerd bewegingswetenschapper te starten op het Impulse project. Tijdens het gehele promotietraject heb je mij vertrouwen gegeven in mijn eigen kunnen en mij begeleid in de keuzes die gemaakt moesten worden. Ook de buitenlandse congressen die we samen hebben bezocht hebben grote indruk op mij gemaakt!

Prof. dr. IJzerman, beste Maarten, ik dank je voor je deskundige inhoudelijke begeleiding, de belangrijke bijdrage die je hebt geleverd aan mijn artikelen en de prettige samenwerking.

Overige leden van de promotiecommissie: Prof. dr. Jane Burridge, prof. dr. Gert Kwakkel, prof. dr. ir. Peter Veltink, prof. dr. Hans Rietman en dr. Rik Buschman, I am honoured to have you on my doctorate board, thank you all very much for your willingness to evaluate my dissertation.

Een bijzonder woord van dank ben ik verschuldigd aan alle deelnemende patiënten. De meesten van hen kwamen van ver en offerden voor iedere meetsessie een hele dag op. Jullie motivatie en inzet waren cruciaal in de totstandkoming van deze promotie.

Dank ben ik ook verschuldigd aan een aantal oud-collega's welke mij, voorafgaand aan het promotietraject, geïntroduceerd hebben in het onderwerp van functionele elektrostimulatie. Rik Buschman, jij begeleidde de patiënten welke een implantaat hadden ontvangen in de pilot studie en liet mij meekijken bij hun bezoeken aan RRD. Zo kon ik op een ongedwongen en plezierige manier ervaring opdoen, welke later goed van pas kwam tijdens de uitvoering van de RCT. Hans van der Aa, als neurochirurg gaf jij mij de kans diverse operaties van deelnemende patiënten bij te wonen. Je straalde altijd enthousiasme uit en de samenwerking met jouw team, welke van essentieel belang was voor een goed verloop van de RCT, verliep erg prettig. Een speciaal woord 
van dank ben ik verschuldigd aan Gerrit Bultstra, die helaas deze dag niet meer mee mag maken. Mijn promotie mag gezien worden als een bekroning op zijn pioniers- en hobbywerk. Vanaf het begin van de ontwikkeling van de implanteerbare peroneus stimulator was hij betrokken en de eerste zenders werden door hem ontworpen. Voor elk technisch probleem welke we ondervonden in de pilot studie had hij op korte termijn een geniale oplossing bedacht.

Daarnaast wil ik ook een aantal collega's bedanken welke mij gedurende de uitvoering van de RCT hebben geholpen. Anand Nene, ik wil jou bedanken voor de hulp bij de selectie van de deelnemende patiënten. Vele patiënten werden door jou gezien en het was niet altijd even gemakkelijk sommigen van hen mede te delen dat ze niet geschikt waren voor deelname aan mijn onderzoek. Leendert Schaake, ik wil je in het bijzonder bedanken voor de hulp tijdens de metingen. Helaas verliepen deze, technisch gezien, niet altijd vlekkeloos. Gelukkig wist jij vaak, ter plekke, een oplossing aan te dragen. Karin Groothuis, jou wil ik bedanken voor de deskundige statistische hulp bij de data analyse. Rik Vegt, ik wil je bedanken voor de hulp bij de verwerking van de Vicon data. Daarnaast wil ik mijn medeauteurs bedanken. Jullie hebben er mede voor gezorgd dat ik hier vandaag mijn proefschrift mag verdedigen.

Ook mijn paranimfen wil ik bedanken. Martin Tenniglo, jij was bij veel facetten van het onderzoek betrokken. Naast je rol bij de selectie van de patiënten wil ik je ook bedanken voor je fysiotherapeutische begeleiding van alle deelnemende patiënten, de gezelligheid tijdens de vele (lange) meetsessies en je bijdrage aan de workshop en het symposium. Ook je belangstelling op persoonlijk vlak heb ik als zeer waardevol ervaren. Hopelijk komen er in de toekomst nog meer gezamenlijke projecten op ons pad. Janine van Til, jou wil ik bedanken voor het leuke contact, zowel binnen als buiten het werk. Er waren veel overeenkomsten te vinden in de levensfases welke we hierin doormaakten. Ondanks dat je momenteel bij de Universiteit Twente werkt, zien/ spreken we elkaar gelukkig nog regelmatig.

Ook dank aan alle collega's van RRD voor hun collegialiteit en gezelligheid. Jullie dragen erg bij aan mijn werkplezier! Een aantal van hen wil ik in het bijzonder bedanken. Rianne Huis in ' $t$ Veld, Brigitte Benerink, Corien Simons, Inger Makaske, Gerdienke Prange en Birgit Molier, jullie wil ik bedanken voor de leuke dagelijkse 
gesprekken, zowel werk als niet-werk gerelateerd. Rianne, in verband met jouw zwangerschapsverlof ben je vandaag waarschijnlijk niet in staat bij mijn promotie aanwezig te zijn. Ik hoop dat je een bijzondere tijd hebt thuis en dat je erg geniet van je moederrol. Miriam Vollenbroek wil ik ook persoonlijk bedanken. Door jou ben ik bij RRD terecht gekomen. Daarnaast heb ik gedurende mijn promotie vele gezellige retourtjes van Geesteren naar Enschede samen met jou mogen afleggen. Marjan Volkering, jou wil ik bedanken voor de hulp bij de organisatie van de workshop en het symposium. Jaap Buurke, ik wil jou bedanken voor de begeleiding tijdens de afronding van mijn promotietraject en voor de hulp bij de organisatie van de workshop en het symposium. Jouw klinisch-wetenschappelijke blik kwam bij beide goed van pas. Ik hoop dat we in de toekomst nog veel projecten samen mogen uitvoeren.

Ook gaat mijn dank uit naar een aantal oud-collega's. Victorien Erren en Carola Mes, met jullie beiden heb ik het grootste gedeelte van mijn promotietraject de kamer mogen delen. Bedankt voor het uitwisselen van alle positieve en negatieve ervaringen welke langskwamen tijdens mijn promotieonderzoek. Michiel Jannink, ondanks onze korte samenwerking, wil ik je bedanken voor de plezierige begeleiding tijdens de afronding van mijn promotietraject.

Natuurlijk mag ik in mijn dankwoord mijn familie niet vergeten! Pap en mam, bedankt voor jullie onvoorwaardelijke steun en vertrouwen in mij. Ik ben jullie intens dankbaar voor alles wat jullie voor mij deden en nog steeds doen. Het is altijd heerlijk om weer 'thuis' te komen. Jan en Marieke, ik ben erg trots dat jullie mijn broer en zus zijn! Ook wil ik mijn schoonouders, schoonzussen en zwagers bedanken voor alles.

Tenslotte, Hanne en Eva, door jullie geboorte is mijn promotiedatum enkele keren uitgesteld. En bijna was het ons derde Kottinkje op komst weer gelukt! Echter, het moeder mogen zijn blijft voor mij de meest speciale promotie. Daar zal mijn wetenschappelijke promotie nooit of te nimmer tegen op kunnen! Hanne en Eva, jullie zijn mij het allerdierbaarst, samen natuurlijk met jullie papa! Thomas, het is fijn om te weten dat je er altijd voor me bent. Ik wil jullie bedanken voor de bijzondere momenten die ik iedere dag weer met jullie 3-en mag beleven! 



\section{Over de auteur}

Anke Kottink-Hutten werd geboren op 28 januari 1979 te Geesteren (Ov). De middelbare school volgde zij in Almelo aan de St.-Canisius, waar zij in 1997 haar VWO diploma behaalde. In datzelfde jaar begon zij haar opleiding Biomedische Gezondheids Wetenschappen (BGW) aan de Katholieke Universiteit Nijmegen, met als afstudeerrichting Bewegingswetenschappen. In april 2001 startte zij haar afstudeerstage bij Roessingh Research and Development (RRD), waarin onderzoek werd gedaan naar de rustmomenten in vier nek/schouderspieren tijdens computerwerkzaamheden bij gezonde proefpersonen en patiënten met Repetitive Strain Injury (RSI). In oktober 2001 werd met deze stage de studie BGW afgerond. Aansluitend is ze als wetenschappelijk onderzoeker bij RRD binnen het cluster 'Restoration of Human Function' aangesteld op het Europese Impulse project. Van november 2005 t/m december 2007 is zij tevens als onderzoeker werkzaam geweest bij de Ziekenhuis Groep Twente, locatie Almelo, op het gebied van de diabetische voet.

Momenteel is zij betrokken bij diverse projecten waarbij Virtual Reality en robotica worden ingezet voor de revalidatie van de armfunctie na een CVA. 



\section{Progress Range}

The following publications have also been published in the Progress range by Roessingh Research and Development, Enschede, the Netherlands. Copies can be ordered, when available, via info@rrd.nl.

1. Pot JWGA, Boer H, van Harten WH, Hermens HJ, Seydel ER. Comprehensive Need-Assessment. Ontwikkeling van een meetinstrument voor zorgbehoeften en kwaliteitsbeoordeling door patiënten. Roessingh Research and Development, The Netherlands, September 1994, ISBN 90-25452-01-2

2. Van Leerdam NGA, Hermens HJ. Revalidatietechnologie in Euregio. Roessingh Research and Development, The Netherlands, July 1995, ISBN 90-75452-02-0

3. Duda L, van Noort LO, Röseler S, Greitemann BOL, van Harten WH, Klazinga NS. Rehabilitation in Germany and the Netherlands, A comparison of two rehabilitation-systems. Roessingh Research and Development, The Netherlands, August 1995, ISBN 90-75452-03-9

4. Hermens HJ, Nene AV, Zilvold G. Electrophysiological Kinesiology. Proceedings of the 11th congress of the International Society of Electrophysiology and Kinesiology in Enschede, Roessingh Research and Development, The Netherlands, October 1996, ISBN 90-75452-04-7

5. Van Harten WH. Bouwen aan een kwaliteitssysteem in de revalidatiezorg. Een poging tot constructieve technology assessment van een kwaliteitssysteem in een gezondheids-zorginstelling. Roessingh Research and Development, The Netherlands, December 1997, ISBN 90-75452-07-1

6. Baardman G, IJzerman MJ. Design and evaluation of a hybrid orthosis for people with paraplegia. Roessingh Research and Development, The Netherlands, November 1997, ISBN 90-75452-08-X

7. Hutten MMR. Lumbar Dynamometry: A useful method for assessment of patients with chronic low back pain? Roessingh Research and Development, The Netherlands, November 1999, ISBN 90-75452-13-6 
8. Van der Salm A, van Harten WH, Maathuis CGB. Ketenkwaliteit Cerebrale Parese Zorg. Een beschrijving van de cerebrale parese zorg en mogelijke verbeteringen hierin. Roessingh Research and Development, The Netherlands, April 2001, ISBN 90-75452-19-5

9. Nederhand MJ. Muscle activation patterns in post traumatic neck pain. Roessingh Research and Development, The Netherlands, March 2003, ISBN 90 75452-27-6

10. Jannink MJA. Usabillity of custom-made orthopaedic shoes in patients with degenerative disorders of the foot. Roessingh Research and Development, the Netherlands, September 2004, ISBN 90-75452-28-4.

11. Blokhorst M. State-dependent factors and attention in whiplash associated disorder. Roessingh Research and Development, The Netherlands, December 2005, ISBN 90-365-2140-8

12. Buurke JH. Walking after stroke; co-ordination patterns \& functional recovery. Roessingh Research and Development, The Netherlands, February 2005, ISBN 90-365-2140-8

13. Van der Salm A. Spasticity reduction using electrical stimulation in the lower limb of spinal cord injury patients. Roessingh Research and Development, The Netherlands, October 2005, ISBN 90-365-2253-6

14. Snoek GJ. Patient preferences for reconstructive interventions of the upper limb in tetraplegia. Roessingh Research and Development, The Netherlands, December 2005, ISBN 90-365-2255-2

15. De Kroon J. Therapeutic electrical stimulation of the upper extremity in stroke. Roessingh Research and Development, The Netherlands, December 2005, ISBN 90-365-2269-2

16. Van Dijk H. Motor skill learning, age and augmented feedback. Roessingh Research and Development, The Netherlands, March 2006, ISBN 90-365-2302-9

17. Mes CAJ. Improving non-optimal results in chronic pain treatment. Roessingh Research and Development, The Netherlands, January 2007, ISBN 90-365-2435-0 
18. Voerman GE. Musculoskeletal neck-shoulder pain: a new ambulant myofeedback intervention approach. Roessingh Research and Development, The Netherlands, March 2007, ISBN 90-365-2460-1

19. Kallenberg LAC. Multi-channel array EMG in chronic neck-shoulder pain. Roessingh Research and Development, The Netherlands, March 2007, ISBN 90365-2459-8

20. Huis in 't Veld MHA. Work-related neck-shoulder pain: The role of cognitivebehavioural factors and remotely supervised treatment. Roessingh Research and Development, The Netherlands, December 2007, ISBN 978-90-365-2584-8

21. Fleuren JFM. Assessment of spasticity - from EMG to patients' perception. Roessingh Research and Development, The Netherlands, October 2009, ISBN 978-90-365-2869-6

22. Reenalda J. Dynamic sitting to prevent pressure ulcers in spinal cord injured. Roessingh Research and Development, The Netherlands, October 2009, ISBN 978-90-365-2884-9

23. Prange GB. Rehabilitation robotics - stimulating restoration of arm function after stroke. Roessingh Research and Development, The Netherlands, October 2009, ISBN 978-90-365-2901-3

24. Vos-van der Hulst M. Prognostic factors and underlying mechanisms in chronic low back pain. Roessingh Research and Development, The Netherlands, November 2009, ISBN 978-90-365-2881-8

25. Kottink-Hutten AIR. Assessment of a two-channel implantable peroneal nerve stimulator post-stroke. Roessingh Research and Development, The Netherlands, February 2010, ISBN 978-90-365-2959-4 